\title{
An inquiry into various aspects of clozapine use
}

\author{
Citation for published version (APA):
}

van der Zalm, Y. C. (2021). An inquiry into various aspects of clozapine use: prescription, monitoring and mortality. [Doctoral Thesis, Maastricht University]. Maastricht University. https://doi.org/10.26481/dis.20210618yz

Document status and date:

Published: 01/01/2021

DOI:

10.26481/dis.20210618yz

Document Version:

Publisher's PDF, also known as Version of record

\section{Please check the document version of this publication:}

- A submitted manuscript is the version of the article upon submission and before peer-review. There can be important differences between the submitted version and the official published version of record.

People interested in the research are advised to contact the author for the final version of the publication, or visit the DOI to the publisher's website.

- The final author version and the galley proof are versions of the publication after peer review.

- The final published version features the final layout of the paper including the volume, issue and page numbers.

Link to publication

\footnotetext{
General rights rights.

- You may freely distribute the URL identifying the publication in the public portal. please follow below link for the End User Agreement:

www.umlib.nl/taverne-license

Take down policy

If you believe that this document breaches copyright please contact us at:

repository@maastrichtuniversity.nl

providing details and we will investigate your claim.
}

Copyright and moral rights for the publications made accessible in the public portal are retained by the authors and/or other copyright owners and it is a condition of accessing publications that users recognise and abide by the legal requirements associated with these

- Users may download and print one copy of any publication from the public portal for the purpose of private study or research.

- You may not further distribute the material or use it for any profit-making activity or commercial gain

If the publication is distributed under the terms of Article $25 \mathrm{fa}$ of the Dutch Copyright Act, indicated by the "Taverne" license above, 


\section{An inquiry into various aspects of clozapine use: prescription, monitoring and mortality.}

Yvonne van der Zalm 
ISBN: 978-94-6423-220-2

Cover design: Marc van der Geer

Layout and printing: ProefschriftMaken | www.proefschriftmaken.nl

Copyright: Yvonne van der Zalm, 2021, the Netherlands 


\title{
An inquiry into various aspects of clozapine use: prescription, monitoring and mortality.
}

\author{
ACADEMISCH PROEFSCHRIFT \\ ter verkrijging van de graad van doctor \\ aan de Universiteit Maastricht \\ op gezag van de Rector Magnificus \\ Prof. dr. Rianne M. Letschert \\ volgens het besluit van het College van Decanen \\ in het openbaar te verdedigen \\ op vrijdag 18 juni om 12.00 uur \\ door
}

Yvonne Catharina van der Zalm 


\section{Promotores}

Prof. dr. J.P. Selten

Prof. dr. I.E. Sommer (Universitair Medisch Centrum Groningen)

\section{Copromotores:}

Dr. P.F.J. Schulte (GGZ Noord-Holland Noord)

Dr. F. Termorshuizen (GGZ Rivierduinen)

\section{Beoordelingscommissie}

Prof. dr. T.A.M.J. van Amelsvoort (voorzitter)

Dr. M. Bak

Dr. S.R.T. Bond-Veerman (GGZ Noord-Holland Noord)

Prof. dr. P.N. van Harten

Prof. dr. B. van Meijel (Vrije Universiteit Amsterdam)

The research presented in this thesis was conducted at the School for Mental Health and Neuroscience (MHeNS), Department of Psychiatry and Neuropsychology of Maastricht University and Rivierduinen Institute for Mental Health Care 




\section{Table of contents}

Chapter $1 \quad$ Introduction 9

PART I Clozapine prescription and monitoring 29

Chapter $2 \quad$ Prescription and underprescription of clozapine in Dutch 31 ambulatory care.

Chapter $3 \quad$ Delegating clozapine monitoring to advanced nurse 51 practitioners: an exploratory, randomized study to assess the effect on prescription and its safety.

$\begin{array}{ll}\text { PART II Clozapine and mortality } & 73\end{array}$

Chapter $4 \quad$ Concerns about bias in studies on clozapine and mortality. $\quad 75$

Chapter $5 \quad$ Use of cardiovascular and antidiabetic drugs before and $\quad 85$ after starting with clozapine versus other antipsychotic drugs: a Dutch database study.

Chapter $6 \quad$ Clozapine and mortality: a comparison with other antipsychotics in a nationwide Danish cohort study.

PART III General discussion

Chapter 7 General discussion

Nederlandse samenvatting $\quad 159$

$\begin{array}{ll}\text { Impactparagraaf } & 165\end{array}$

List of publications 169

Dankwoord 171

$\begin{array}{ll}\text { Curriculum Vitae } & 177\end{array}$ 

Chapter 1 
This introduction provides a brief background on schizophrenia and the treatment thereof, on the antipsychotic drug clozapine and on the association between clozapine and mortality. Furthermore, this chapter provides a rationale for the research conducted. At the end of this chapter, the aims and outline of this thesis are presented.

\section{Schizophrenia}

Schizophrenia is a disabling mental disorder with a median point prevalence of $0.46 \%$ (Saha, Chant, Welham, \& McGrath, 2005), with large differences between studies and countries (Jongsma et al., 2018; Saha et al., 2005). Schizophrenia is characterized by: (a) positive symptoms, which are delusions and hallucinations; (b) disorganized speech; (c) grossly disorganized or catatonic behavior; and (d) negative symptoms, such as diminished emotional expression, avolition, poverty of speech and social withdrawal (Tandon et al., 2013). Another diagnostic criterion of schizophrenia is social or occupational dysfunction (Tandon et al., 2013), which can be very burdening to patients and their relatives. In addition, this disorder is also associated with a significant economic burden (Cloutier et al., 2016).

\section{Antipsychotic treatment}

The core of treatment for schizophrenia consists of antipsychotic drugs, which are mainly effective in the treatment of positive symptoms (Aleman et al., 2017; Veerman, Schulte, \& de Haan, 2017). Antipsychotics can roughly be divided in First Generation Antipsychotics (FGAs) and Second Generation Antipsychotics (SGAs). FGAs were first introduced in the fifties, while most SGAs were introduced after 1990. In general, SGAs have different side-effects, compared to FGAs as a result of different receptor binding profiles (Siafis, Tzachanis, Samara, \& Papazisis, 2018). FGAs predominantly bind to dopamine 2 receptors and, therefore, have a high probability of causing extrapyramidal side-effects (acute dystonia, akathisia, parkinsonism and tardive dyskinesia). Most SGAs bind to multiple receptors, in many cases resulting in a greater risk of metabolic sideeffects (Leucht et al., 2013).

\section{Treatment-resistant schizophrenia}

A part of the patients are treatment-resistant or become treatment-resistant in the course of their treatment trajectory: they continue to suffer from positive symptoms, despite of treatment with antipsychotics. Various definitions of treatment-resistance have been used 
in the past, but in a recent consensus guideline (Howes et al., 2017), treatment-resistant schizophrenia (TRS) is defined as the persistence of at least moderately severe symptoms for more than 12 weeks, despite two adequate trials with different antipsychotics. A trial with a drug is considered to be adequate if the dosage was equivalent to $600 \mathrm{mg}$ of chlorpromazine or more, if this dosage was used for 6 weeks or more, and if during that time, at least $80 \%$ of the prescribed doses was taken (Howes et al., 2017).

The long existing lack of consensus on treatment resistance caused a large variability in reported prevalence rates, but it is generally accepted that a third of patients with schizophrenia is treatment-resistant (Conley \& Kelly, 2001; Kennedy, Altar, Taylor, Degtiar, \& Hornberger, 2014; Stroup, Gerhard, Crystal, Huang, \& Olfson, 2014). In $70 \%$ of TRS patients, TRS is present from onset of illness (Lally et al., 2016; Lally \& Gaughran, 2018).

\section{Clozapine}

Clozapine was the first SGA, as it was developed in the fifties. However, after several fatalities in Finland due to clozapine-induced agranulocytosis (first described in 1975 (Griffith \& Saameli, 1975)), it was withdrawn from the market. In subsequent years no new drugs were discovered that were just as effective as clozapine for patients with TRS. After the landmark study of Kane (Kane, Honigfeld, Singer, \& Meltzer, 1988) it was reintroduced in the nineties. This re-introduction was possible thanks to the implementation of weekly neutrophil counts during the first months of use

Clozapine is the only approved antipsychotic for treatment resistant schizophrenia and it is effective for 40 to $70 \%$ of patients with TRS (Agid et al., 2011; Chakos, Lieberman, Hoffman, Bradford, \& Sheitman, 2001; Essali, Al-Haj Haasan, Li, \& Rathbone, 2009; Kane et al., 1988; Siskind, McCartney, Goldschlager, \& Kisely, 2016; Souza, Kayo, Tassell, Martins, \& Elkis, 2013; Wahlbeck, Cheine, \& Essali, 2000). In international guidelines (Group, 2019; "NICE Guidance, Quality statement 4: Treatment with clozapine.," 2015), clozapine is the preferred treatment for these patients, if trials with 2 other antipsychotics have shown to be ineffective (Hasan et al., 2012; Lehman et al., 2004; "NICE Guidance, Quality statement 4: Treatment with clozapine.," 2015). Most systematic reviews conclude that clozapine is superior to other antipsychotics in patients with TRS. However, a recent review by Samara et al. (Samara et al., 2016) did not report differences in efficacy between clozapine and other antipsychotics. Siskind et al (Siskind et al., 2016), who conducted a comparable review, did find clozapine to be superior to other antipsychotics. The authors of the latter study explained that the results obtained by Samara et al. were insufficiently controlled for both funding source 
and dosage. In addition, the results were also affected by the outcome of both positive and negative symptoms, while it is known that antipsychotics are hardly effective for negative symptoms. Some reviews even found clozapine to be more effective in patients with non-resistant schizophrenia too (Huhn et al., 2019) and suggested that clozapine may also be more effective than other antipsychotics when used as first- or second-line treatment (Okhuijsen-Pfeifer et al., 2018). Although it is known that the abundant receptor binding profile of clozapine differs from that of other antipsychotics (Siafis et al., 2018), the working mechanism of this drug is still not elucidated (Seeman, 2014).

It is important to note that compliance with clozapine is better than with other antipsychotics (Cooper, Moisan, \& Gregoire, 2007) and it is also a cost-effective treatment for patients with TRS (Hayhurst, Brown, \& Lewis, 2002; Revicki, 2000; Seshamani, 2002).

\section{Other indications for clozapine}

Apart from TRS, there are other indications for the use of clozapine, as it has been found to reduce suicidality (Hennen \& Baldessarini, 2005; Meltzer et al., 2003), aggression (Frogley, Taylor, Dickens, \& Picchioni, 2012; Krakowski, Czobor, Citrome, Bark, \& Cooper, 2006; Volavka et al., 2004), tardive dyskinesia (TD) (Hazari, Kate, \& Grover, 2013; Lee, Baek, Bae, Choi, \& Hong, 2019), extrapyramidal symptoms (EPS) (Leucht et al., 2013) and substance use (Kelly, Daley, $\&$ Douaihy, 2012) in patients with psychotic disorders. International guidelines are not consistent with regard to other indications than TRS. For example, clozapine has been approved in the USA for reducing the risk of recurrent suicidal behavior in patients with schizophrenia or schizoaffective disorder, but not in the European Union. At the same time, it has been registered in many European countries for acute EPS and TD in case of failure of the usual therapeutic strategy, but not in the USA. The Dutch guideline for clozapine recommends it for patients with a non-affective psychotic disorder who suffer from treatment-resistant positive or negative symptoms, persistent aggressive or suicidal behavior, untreatable EPS or TD and substance use disorder ("Dutch Clozapine Collaboration Group. Guideline for the use of clozapine [English version]," 2009). This guideline also recommends considering clozapine for psychotic disorder in Parkinson's disease, for treatment-resistant psychosis in bipolar disorder or depression and for aggression or suicidality in patients with a borderline personality disorder. 


\section{Side-effects and monitoring}

When clozapine was re-introduced in the nineties, it could only be prescribed under the condition of an intensive monitoring of white blood cells to manage the risk of agranulocytosis. There are different regulations in different countries (Nielsen et al., 2016), but they all have weekly neutrophil counts in the first months of the initiation phase in common. The duration of this period of weekly lab exams differs between countries. In the Netherlands and most European countries, this period is 18 weeks. In the USA and Japan, this period is 26 weeks (Nielsen et al., 2016). There are also differences in the degree of obligation of these exams between countries. In Iceland for example, the monitoring is not mandatory (Ingimarsson, MacCabe, Haraldsson, Jonsdottir, \& Sigurdsson, 2016), but in the USA and the UK, the prescription of clozapine is only possible after the check of the neutrophils. In the Netherlands, the lab exams are mandatory. However, there is no verification of the performance of the neutrophil counts (Nielsen et al., 2016).

Apart from agranulocytosis, there are other side-effects which demand attention. Although the monitoring of other side-effects is not mandatory in most countries (Nielsen et al., 2016), some can be lethal, for example ileus (West, Rowbotham, Xiong, \& Kenedi, 2017), pneumonia (De Leon, Sanz, \& De Las Cuevas, 2020), thrombo-embolism (Poudyal \& Lohani, 2019) and myocarditis (Siskind et al., 2020). Apart from these acute life threatening side-effects, there are other side-effects that can shorten patients' life-expectancy or are very bothersome, for example salivation, sedation, constipation, weight gain, hypotension, tachycardia and urinary problems. These side-effects are most prominent in the titration phase, but may persist during its use thereafter. Considering that clozapine is often a drug of last resort, it is important to continue its use until it is clear whether it is effective or not. Therefore, close monitoring of all side-effects and where necessary treatment thereof, might prevent premature termination.

\section{Prescription rates}

The desirable clozapine prescription rate can be estimated, based on proportions of TRS. If a third of patients with schizophrenia is treatment resistant and clozapine is effective in $40 \%$ of them (Siskind et al., 2016), clozapine prescription rates among patients with schizophrenia should be approximately 13\%, based on TRS alone. Given other indications, the desired proportion may be close to $20 \%$. However, in many countries, clozapine is still under-prescribed (Bachmann et al., 2017). Studies of clozapine prescription rates show large differences between countries. For instance, rates of $1.2 \%, 4.8 \%$ and $10.1 \%$ were found in France (Verdoux et al., 2016), the USA 
(Olfson, Gerhard, Crystal, \& Stroup, 2016) and Denmark (Nielsen, Roge, Schjerning, Sorensen, \& Taylor, 2012) respectively. In the Netherlands, the proportion of patients on clozapine is unknown. In addition to the under-prescription of clozapine, the delay before it is prescribed is also a cause for concern. There is emerging evidence that treatment resistance can be present in the earliest stages of psychosis and can therefore be diagnosed within the first months after onset of psychosis (Agid et al., 2011; Lally et al., 2016). However, if clozapine is prescribed, it is often preceded by an unnecessary and unwarranted delay. For example, this delay in London was on average 4 years (Howes et al., 2012) and this seems to be common practice in other countries as well (Grover, Hazari, Chakrabarti, \& Avasthi, 2015; Nielsen et al., 2012; Ucok et al., 2015; Wheeler, 2008). A delay does not only prolong the patients' suffering, but it may also diminish the effect of clozapine (Ucok et al., 2015).

\section{Causes of under-prescription and delay}

There are many causes for the underprescription of clozapine or the delay before prescribing this drug (Thien \& O’Donoghue, 2018; Verdoux, Quiles, Bachmann, \& Siskind, 2018). Important barriers for prescribers are the concern about the adverse effects of clozapine and the required blood monitoring. This monitoring is very timeconsuming and some psychiatrists do not have sufficient time to monitor carefully and accurately. Prescribers often state that despite of the guidelines, they would rather prescribe a third or fourth antipsychotic drug or try polypharmacy before they would prescribe clozapine. Other frequently mentioned barriers are a lack of prescriber knowledge and confidence. In addition, local traditions and prescribers preferences are reasons for under-prescription too (Thien \& O'Donoghue, 2018; Verdoux et al., 2018). It is important to address this reluctance to prescribe clozapine, earlier described as 'prescribers fear', because it withholds patients from an effective, evidence-based treatment (Cohen, 2014).

Although prescribers assume that the side-effects and the blood monitoring are barriers for patients too, only a minority of patients were found to dislike clozapine due to regular blood monitoring or side-effects (Angermeyer, Loffler, Muller, Schulze, \& Priebe, 2001; S. H. Gee, Shergill, \& Taylor, 2017). The most important barrier for patients is a possible hospital admission (S. H. Gee et al., 2017). 


\section{Improving prescription}

Although underprescription of clozapine is an international problem, research on improving prescription rates is scarce. No randomized controlled trial has been done yet to study the effects of an intervention designed to improve clozapine prescription rates. However, there have been initiatives to improve clozapine prescription rates by improving education and information, as for example internet-based educational programs in New York (Carruthers et al., 2016) and the founding of a national expert group in the Netherlands (Bogers, Schulte, Van Dijk, Bakker, \& Cohen, 2016). There are a few other initiatives to improve clozapine prescription rates. The first is the utilization of nurse-led clinics as a part of the clozapine management system in Australia (Clark, Wilton, Baune, Procter, \& Hustig, 2014) and the second is the establishment of specialized teams for the identification and treatment of patients with treatmentresistant schizophrenia in London (Beck et al., 2014). In Australia, the nurse-led clinics led to an increase in timely monitoring. However, the nurses only coordinated the care for stable clozapine users and not for patients in the initiation phase, when monitoring is most time-consuming. Introducing specialized teams in London, led to an increased clozapine use. Nevertheless, the authors acknowledged that there also are disadvantages in deploying additional teams: an extra service can cause confusion among clinicians and patients about the clinicians' role and responsibilities, and among patients, because of multiple appointments of patients with different teams. This latter initiative is in line with a UK survey that concluded that practitioners perceived the presence of dedicated staff for outpatients clozapine monitoring in the initiation phase, as the factor that would enable the use of clozapine most (S. Gee, Vergunst, Howes, \& Taylor, 2014). An additional advantage of the deployment of, for example, specialized nurses in clozapine monitoring is that nurses maintain a longer therapeutic alliance with patients and their relatives than other mental health professionals do. This is beneficial because a better therapeutic relationship is associated with better adherence to medication among patients with schizophrenia (McCabe et al., 2012).

\section{Mortality and schizophrenia}

In patients with schizophrenia, life expectancy is about 15 years shorter (Hjorthoj, Sturup, McGrath, \& Nordentoft, 2017) compared to the general population and in recent decades, the differential mortality gap associated with schizophrenia has been increasing (Saha, Chant, \& McGrath, 2007). Factors that contribute to this mortality gap are: the negative side-effects of antipsychotics (with metabolic side-effects as the most important of these), unhealthy lifestyle (poor diet, smoking, excess alcohol consumption, and lack of exercise), high risk of suicide and accidents, late detection 
and insufficient treatment of physical illnesses (Laursen, Munk-Olsen, \& Vestergaard, 2012).

\section{Clozapine and mortality}

Given the shortened life expectancy of patients with schizophrenia and the possibly negative influence of antipsychotics, it is important to study the associations with mortality of different antipsychotics with different side-effect profiles. Regarding clozapine, this is even more important, because concerns about mortality due to the potentially dangerous side-effects may also contribute to low prescription rates. Surprisingly, however, where most studies do not find a difference in all-cause mortality between clozapine and other antipsychotics (Hennessy et al., 2002; Ringback Weitoft et al., 2014; Stroup, Gerhard, Crystal, Huang, \& Olfson, 2016; Taipale et al., 2017), three large studies have reported a significant reduction of mortality associated with the use of clozapine (Hayes et al., 2015; Tiihonen et al., 2009; Walker, Lanza, Arellano, \& Rothman, 1997).

\section{Bias in studies on clozapine and mortality}

In the studies on clozapine and mortality there are possible sources of bias that may affect the results. An important source of bias is survivor bias, especially in the analysis of death by suicide. Suicides occur more frequently in the first years after the onset of psychosis (Kuo, Tsai, Lo, Wang, \& Chen, 2005; Melle et al., 2017; Termorshuizen et al., 2013), whereas clozapine is often first prescribed years after onset (Howes et al., 2012; Nielsen et al., 2012). The best way to address this issue is the use of an incidence cohort.

Another potential source of bias is confounding by indication. Clozapine may be prescribed to younger and relatively healthy patients more often, as prescribers may be reluctant to prescribe this drug to patients who already have metabolic or cardiac comorbidity. This could lead to an observation of a lower mortality rate among clozapine users. Another example is the effect of the intensive monitoring of patients that accompanies the use of clozapine. Given that patients with severe mental illness are often somatically undertreated (Laursen, Munk-Olsen, \& Gasse, 2011; Swildens, Termorshuizen, de Ridder, Smeets, \& Engelhard, 2016), clozapine-users may receive more adequate somatic treatment.

Even without the above mentioned sources of bias, it is difficult to compare studies on clozapine and mortality for a number of reasons. First, the comparison groups in the 
studies varied. Use of clozapine was compared, for example, to use of all non-clozapine antipsychotics (Taipale et al., 2020) or to use of all non-clozapine users including those not using any antipsychotic (Hayes et al., 2015; Tiihonen et al., 2009). Second, the diagnostic inclusion criteria varied, e.g. users of antipsychotics irrespective of diagnosis (Gjerden, Slørdal, \& Bramness, 2010), only those with a diagnosis of schizophrenia (Ringback Weitoft et al., 2014) or restricted to patients with TRS (Stroup et al., 2016; Wimberley et al., 2017). Third, some studies were conducted with only incident cases (Kiviniemi, 2013) while others included prevalent cases too (Tiihonen et al., 2009). Fourth, use of clozapine could be defined as any use during the observation period (Hayes et al., 2015), sole use during the observation period (Crump, Winkleby, Sundquist, \& Sundquist, 2013) or intention to treat with clozapine (Wimberley et al., 2017). Therefore, it is difficult to conduct a systematic review or meta-analysis when all studies have different outcomes and inclusion criteria.

\section{Outline and aims}

This thesis is focused on two aspects of clozapine, that are both important in relation to its under-prescription. The first part studies clozapine prescription rates and how to improve these rates. The second part investigates clozapine and mortality. As clozapine is associated with acute and prolonged life-threatening side-effects, the prescribers' fear of side-effects and an increased mortality, may affect the way they prescribe.

The aim of the first part of this thesis was to test whether clozapine prescription rates can be increased. First, clozapine prescription rates in Dutch outpatient teams are presented in Chapter 2. This chapter describes the baseline measurements at the start of a randomized trial to improve prescription rates. The results of this trial are presented in Chapter 3. This trial examined whether psychiatrists prescribe more clozapine if they can delegate the labour-intensive monitoring tasks to an advanced nurse practitioner. As previously discussed, this could be an important factor enabling the prescription of clozapine. In addition to the effect on prescription rates, the safety of the clozapine monitoring in both conditions was assessed.

The aim of the second part of this thesis was to clarify the complicated and debated relationship between clozapine and mortality. This part starts with Chapter 4, a short review on studies on clozapine and mortality. In this chapter, we also commented on a meta-analysis on this subject. Chapter 5 is a Dutch database study to examine possible forms of bias that may have influenced results of other studies. Chapter 6 is a nation-wide Danish cohort study into mortality and clozapine. In this study clozapine is compared to other categories of antipsychotics. Finally, in Chapter 7, I will summarize and discuss the 
findings presented in this thesis. Thereafter the limitations and strengths of the studies are described, followed by the implications for practice and suggestions for future studies. 


\section{References}

Agid, O., Arenovich, T., Sajeev, G., Zipursky, R. B., Kapur, S., Foussias, G., \& Remington, G. (2011). An algorithm-based approach to first-episode schizophrenia: response rates over 3 prospective antipsychotic trials with a retrospective data analysis. J Clin Psychiatry, 72(11), 1439-1444. doi:10.4088/JCP.09m05785yel

Aleman, A., Lincoln, T. M., Bruggeman, R., Melle, I., Arends, J., Arango, C., \& Knegtering, H. (2017). Treatment of negative symptoms: Where do we stand, and where do we go? Schizophr Res, 186, 55-62. doi:10.1016/j.schres.2016.05.015

Angermeyer, M. C., Loffler, W., Muller, P., Schulze, B., \& Priebe, S. (2001). Patients' and relatives' assessment of clozapine treatment. Psychol Med, 31(3), 509-517.

Bachmann, C. J., Aagaard, L., Bernardo, M., Brandt, L., Cartabia, M., Clavenna, A., . . Taylor, D. (2017). International trends in clozapine use: a study in 17 countries. Acta Psychiatr Scand, 136(1), 37-51. doi:10.1111/acps.12742

Beck, K., McCutcheon, R., Bloomfield, M. A., Gaughran, F., Reis Marques, T., MacCabe, J., . . Howes, O. D. (2014). The practical management of refractory schizophrenia--the Maudsley Treatment REview and Assessment Team service approach. Acta Psychiatr Scand, 130(6), 427-438. doi:10.1111/ acps. 12327

Bogers, J. P., Schulte, P. F., Van Dijk, D., Bakker, B., \& Cohen, D. (2016). Clozapine Underutilization in the Treatment of Schizophrenia: How Can Clozapine Prescription Rates Be Improved? J Clin Psychopharmacol, 36(2), 109-111. doi:10.1097/jcp.0000000000000478

Carruthers, J., Radigan, M., Erlich, M. D., Gu, G., Wang, R., Frimpong, E. Y., . . Stroup, T. S. (2016). An Initiative to Improve Clozapine Prescribing in New York State. Psychiatr Serv, 67(4), 369-371. doi:10.1176/appi.ps.201500493

Chakos, M., Lieberman, J., Hoffman, E., Bradford, D., \& Sheitman, B. (2001). Effectiveness of secondgeneration antipsychotics in patients with treatment-resistant schizophrenia: a review and metaanalysis of randomized trials. Am J Psychiatry, 158(4), 518-526. doi:10.1176/appi.ajp.158.4.518

Clark, S. R., Wilton, L., Baune, B. T., Procter, N., \& Hustig, H. (2014). A state-wide quality improvement system utilising nurse-led clinics for clozapine management. Australas Psychiatry, 22(3), 254-259. doi:10.1177/1039856214533395

Cloutier, M., Aigbogun, M. S., Guerin, A., Nitulescu, R., Ramanakumar, A. V., Kamat, S. A., . . Wu, E. (2016). The Economic Burden of Schizophrenia in the United States in 2013. J Clin Psychiatry, 77(6), 764-771. doi:10.4088/JCP.15m10278

Cohen, D. (2014). Prescribers fear as a major side-effect of clozapine. Acta Psychiatr Scand, 130(2), 154155. doi:10.1111/acps. 12294

Conley, R. R., \& Kelly, D. L. (2001). Management of treatment resistance in schizophrenia. Biol Psychiatry, 50(11), 898-911.

Cooper, D., Moisan, J., \& Gregoire, J. P. (2007). Adherence to atypical antipsychotic treatment among newly treated patients: a population-based study in schizophrenia. J Clin Psychiatry, 68(6), 818825. 
Crump, C., Winkleby, M. A., Sundquist, K., \& Sundquist, J. (2013). Comorbidities and mortality in persons with schizophrenia: a Swedish national cohort study. Am J Psychiatry, 170(3), 324-333. doi:10.1176/appi.ajp.2012.12050599

De Leon, J., Sanz, E. J., \& De Las Cuevas, C. (2020). Data From the World Health Organization's Pharmacovigilance Database Supports the Prominent Role of Pneumonia in Mortality Associated With Clozapine Adverse Drug Reactions. Schizophr Bull, 46(1), 1-3. doi:10.1093/schbul/sbz093

Dutch Clozapine Collaboration Group. Guideline for the use of clozapine [English version]. . (2009). Retrieved from http://clozapinepluswerkgroep.nl/pdf/SKMBT_C45110040808460.pdf.

Essali, A., Al-Haj Haasan, N., Li, C., \& Rathbone, J. (2009). Clozapine versus typical neuroleptic medication for schizophrenia. Cochrane Database Syst Rev(1), Cd000059. doi:10.1002/14651858. CD000059.pub2

Frogley, C., Taylor, D., Dickens, G., \& Picchioni, M. (2012). A systematic review of the evidence of clozapine's anti-aggressive effects. Int J Neuropsychopharmacol, 15(9), 1351-1371. doi:10.1017/ s146114571100201x

Gee, S., Vergunst, F., Howes, O., \& Taylor, D. (2014). Practitioner attitudes to clozapine initiation. Acta Psychiatr Scand, 130(1), 16-24. doi:10.1111/acps.12193

Gee, S. H., Shergill, S. S., \& Taylor, D. M. (2017). Patient attitudes to clozapine initiation. Int Clin Psychopharmacol, 32(6), 337-342. doi:10.1097/yic.0000000000000188

Gjerden, P., Slørdal, L., \& Bramness, J. G. (2010). Prescription persistence and safety of antipsychotic medication: a national registry-based 3-year follow-up. Eur J Clin Pharmacol, 66(9), 911-917. doi:10.1007/s00228-010-0839-9

Griffith, R. W., \& Saameli, K. (1975). Letter: Clozapine and agranulocytosis. Lancet, 2(7936), 657. doi:10.1016/s0140-6736(75)90135-x

Group, G. W. (2019). THE AMERICAN PSYCHIATRIC ASSOCIATION PRACTICE GUIDELINE FOR THE TREATMENT OF PATIENTS WITH SCHIZOPHRENIA. Retrieved from https:// www.psychiatry.org > Practice , Clinical Practice Guidelines > APA-D...

Grover, S., Hazari, N., Chakrabarti, S., \& Avasthi, A. (2015). Delay in initiation of clozapine: a retrospective study from a tertiary care hospital in North India. Psychiatry Res, 226(1), 181-185. doi:10.1016/j. psychres.2014.12.046

Hasan, A., Falkai, P., Wobrock, T., Lieberman, J., Glenthoj, B., Gattaz, W. F., . . Moller, H. J. (2012). World Federation of Societies of Biological Psychiatry (WFSBP) Guidelines for Biological Treatment of Schizophrenia, part 1: update 2012 on the acute treatment of schizophrenia and the management of treatment resistance. World J Biol Psychiatry, 13(5), 318-378. doi:10.3109/15622975.2012.69 6143

Hayes, R. D., Downs, J., Chang, C. K., Jackson, R. G., Shetty, H., Broadbent, M., . . Stewart, R. (2015). The effect of clozapine on premature mortality: an assessment of clinical monitoring and other potential confounders. Schizophr Bull, 41(3), 644-655. doi:10.1093/schbul/sbu120

Hayhurst, K. P., Brown, P., \& Lewis, S. W. (2002). The cost-effectiveness of clozapine: a controlled, populationbased, mirror-image study. J Psychopharmacol, 16(2), 169-175. doi:10.1177/026988110201600208

Hazari, N., Kate, N., \& Grover, S. (2013). Clozapine and tardive movement disorders: a review. Asian J 
Psychiatr, 6(6), 439-451. doi:10.1016/j.ajp.2013.08.067

Hennen, J., \& Baldessarini, R. J. (2005). Suicidal risk during treatment with clozapine: a meta-analysis. Schizophr Res, 73(2-3), 139-145. doi:10.1016/j.schres.2004.05.015

Hennessy, S., Bilker, W. B., Knauss, J. S., Margolis, D. J., Kimmel, S. E., Reynolds, R. F., . . Strom, B. L. (2002). Cardiac arrest and ventricular arrhythmia in patients taking antipsychotic drugs: cohort study using administrative data. Bmj, 325(7372), 1070.

Hjorthoj, C., Sturup, A. E., McGrath, J. J., \& Nordentoft, M. (2017). Years of potential life lost and life expectancy in schizophrenia: a systematic review and meta-analysis. Lancet Psychiatry, 4(4), 295301. doi:10.1016/s2215-0366(17)30078-0

Howes, O. D., McCutcheon, R., Agid, O., de Bartolomeis, A., van Beveren, N. J., Birnbaum, M. L., . . . Correll, C. U. (2017). Treatment-Resistant Schizophrenia: Treatment Response and Resistance in Psychosis (TRRIP) Working Group Consensus Guidelines on Diagnosis and Terminology. Am J Psychiatry, 174(3), 216-229. doi:10.1176/appi.ajp.2016.16050503

Howes, O. D., Vergunst, F., Gee, S., McGuire, P., Kapur, S., \& Taylor, D. (2012). Adherence to treatment guidelines in clinical practice: study of antipsychotic treatment prior to clozapine initiation. $\mathrm{Br} J$ Psychiatry, 201(6), 481-485. doi:10.1192/bjp.bp.111.105833

Huhn, M., Nikolakopoulou, A., Schneider-Thoma, J., Krause, M., Samara, M., Peter, N., . . Leucht, S. (2019). Comparative efficacy and tolerability of 32 oral antipsychotics for the acute treatment of adults with multi-episode schizophrenia: a systematic review and network meta-analysis. Lancet, 394(10202), 939-951. doi:10.1016/s0140-6736(19)31135-3

Ingimarsson, O., MacCabe, J. H., Haraldsson, M., Jonsdottir, H., \& Sigurdsson, E. (2016). Neutropenia and agranulocytosis during treatment of schizophrenia with clozapine versus other antipsychotics: an observational study in Iceland. BMC Psychiatry, 16(1), 441. doi:10.1186/s12888-016-1167-0

Jongsma, H. E., Gayer-Anderson, C., Lasalvia, A., Quattrone, D., Mulè, A., Szöke, A., . . . Kirkbride, J. B. (2018). Treated Incidence of Psychotic Disorders in the Multinational EU-GEI Study. JAMA Psychiatry, 75(1), 36-46. doi:10.1001/jamapsychiatry.2017.3554

Kane, J., Honigfeld, G., Singer, J., \& Meltzer, H. (1988). Clozapine for the treatment-resistant schizophrenic. A double-blind comparison with chlorpromazine. Arch Gen Psychiatry, 45(9), 789-796.

Kelly, T. M., Daley, D. C., \& Douaihy, A. B. (2012). Treatment of substance abusing patients with comorbid psychiatric disorders. Addict Behav, 37(1), 11-24. doi:10.1016/j.addbeh.2011.09.010

Kennedy, J. L., Altar, C. A., Taylor, D. L., Degtiar, I., \& Hornberger, J. C. (2014). The social and economic burden of treatment-resistant schizophrenia: a systematic literature review. Int Clin Psychopharmacol, 29(2), 63-76. doi:10.1097/YIC.0b013e32836508e6

Kiviniemi, M., J. Suvisaari, H. Koivumaa-Honkanen, U. Häkkinen, M. Isohanni, H. Hakkoe. (2013). Antipsychotics and mortality in first-onset schizophrenia: Prospective Finnish register study with 5-year follow-up. Schizophr Res, 150, 274-280.

Krakowski, M. I., Czobor, P., Citrome, L., Bark, N., \& Cooper, T. B. (2006). Atypical antipsychotic agents in the treatment of violent patients with schizophrenia and schizoaffective disorder. Arch Gen Psychiatry, 63(6), 622-629. doi:10.1001/archpsyc.63.6.622

Kuo, C. J., Tsai, S. Y., Lo, C. H., Wang, Y. P., \& Chen, C. C. (2005). Risk factors for completed suicide in 
schizophrenia. J Clin Psychiatry, 66(5), 579-585.

Lally, J., Ajnakina, O., Di Forti, M., Trotta, A., Demjaha, A., Kolliakou, A., . . Murray, R. M. (2016).

Two distinct patterns of treatment resistance: clinical predictors of treatment resistance in firstepisode schizophrenia spectrum psychoses. Psychol Med, 46(15), 3231-3240. doi:10.1017/ s0033291716002014

Lally, J., \& Gaughran, F. (2018). Treatment resistant schizophrenia - review and a call to action. Ir J Psychol Med, 1-13. doi:10.1017/ipm.2018.47

Laursen, T. M., Munk-Olsen, T., \& Gasse, C. (2011). Chronic somatic comorbidity and excess mortality due to natural causes in persons with schizophrenia or bipolar affective disorder. PLoS One, 6(9), e24597. doi:10.1371/journal.pone.0024597

Laursen, T. M., Munk-Olsen, T., \& Vestergaard, M. (2012). Life expectancy and cardiovascular mortality in persons with schizophrenia. Curr Opin Psychiatry, 25(2), 83-88. doi:10.1097/ YCO.0b013e32835035ca

Lee, D., Baek, J. H., Bae, M., Choi, Y., \& Hong, K. S. (2019). Long-Term Response to Clozapine and Its Clinical Correlates in the Treatment of Tardive Movement Syndromes: A Naturalistic Observational Study in Patients With Psychotic Disorders. J Clin Psychopharmacol, 39(6), 591-596. doi:10.1097/ jcp.0000000000001114

Lehman, A. F., Lieberman, J. A., Dixon, L. B., McGlashan, T. H., Miller, A. L., Perkins, D. O., \& Kreyenbuhl, J. (2004). Practice guideline for the treatment of patients with schizophrenia, second edition. Am J Psychiatry, 161(2 Suppl), 1-56.

Leucht, S., Cipriani, A., Spineli, L., Mavridis, D., Orey, D., Richter, F., .. Davis, J. M. (2013). Comparative efficacy and tolerability of 15 antipsychotic drugs in schizophrenia: a multiple-treatments metaanalysis. Lancet, 382(9896), 951-962. doi:10.1016/s0140-6736(13)60733-3

McCabe, R., Bullenkamp, J., Hansson, L., Lauber, C., Martinez-Leal, R., Rossler, W., . . Priebe, S. (2012). The therapeutic relationship and adherence to antipsychotic medication in schizophrenia. PLoS One, 7(4), e36080. doi:10.1371/journal.pone.0036080

Melle, I., Olav Johannesen, J., Haahr, U. H., Ten Velden Hegelstad, W., Joa, I., Langeveld, J., . . Friis, S. (2017). Causes and predictors of premature death in first-episode schizophrenia spectrum disorders. World Psychiatry, 16(2), 217-218. doi:10.1002/wps.20431

Meltzer, H. Y., Alphs, L., Green, A. I., Altamura, A. C., Anand, R., Bertoldi, A., . . Potkin, S. (2003). Clozapine treatment for suicidality in schizophrenia: International Suicide Prevention Trial (InterSePT). Arch Gen Psychiatry, 60(1), 82-91.

NICE Guidance, Quality statement 4: Treatment with clozapine. (2015, February 2015. ). Retrieved from https:/www.nice.org.uk/guidance/qs80/chapter/quality-statement-4-treatment-with-clozapine

Nielsen, J., Roge, R., Schjerning, O., Sorensen, H. J., \& Taylor, D. (2012). Geographical and temporal variations in clozapine prescription for schizophrenia. Eur Neuropsychopharmacol, 22(11), 818-824. doi:10.1016/j.euroneuro.2012.03.003

Nielsen, J., Young, C., Ifteni, P., Kishimoto, T., Xiang, Y. T., Schulte, P. F., . . Taylor, D. (2016). Worldwide Differences in Regulations of Clozapine Use. CNS Drugs, 30(2), 149-161. doi:10.1007/s40263016-0311-1 
Okhuijsen-Pfeifer, C., Huijsman, E. A. H., Hasan, A., Sommer, I. E. C., Leucht, S., Kahn, R. S., \& Luykx, J. J. (2018). Clozapine as a first- or second-line treatment in schizophrenia: a systematic review and meta-analysis. Acta Psychiatr Scand, 138(4), 281-288. doi:10.1111/acps.12954

Olfson, M., Gerhard, T., Crystal, S., \& Stroup, T. S. (2016). Clozapine for Schizophrenia: State Variation in Evidence-Based Practice. Psychiatr Serv, 67(2), 152. doi:10.1176/appi.ps.201500324

Poudyal, R., \& Lohani, S. (2019). Clozapine associated pulmonary embolism: systematic review. J Community Hosp Intern Med Perspect, 9(4), 300-304. doi:10.1080/20009666.2019.1627848

Revicki, D. A. (2000). The new atypical antipsychotics: a review of pharmacoeconomic studies. Expert Opin Pharmacother, 1(2), 249-260. doi:10.1517/14656566.1.2.249

Ringback Weitoft, G., Berglund, M., Lindstrom, E. A., Nilsson, M., Salmi, P., \& Rosen, M. (2014). Mortality, attempted suicide, re-hospitalisation and prescription refill for clozapine and other antipsychotics in Sweden-a register-based study. Pharmacoepidemiol Drug Saf, 23(3), 290-298. doi: $10.1002 /$ pds.3567

Saha, S., Chant, D., \& McGrath, J. (2007). A systematic review of mortality in schizophrenia: is the differential mortality gap worsening over time? Arch Gen Psychiatry, 64(10), 1123-1131. doi:10.1001/archpsyc.64.10.1123

Saha, S., Chant, D., Welham, J., \& McGrath, J. (2005). A systematic review of the prevalence of schizophrenia. PLoS Med, 2(5), e141. doi:10.1371/journal.pmed.0020141

Samara, M. T., Dold, M., Gianatsi, M., Nikolakopoulou, A., Helfer, B., Salanti, G., \& Leucht, S. (2016). Efficacy, Acceptability, and Tolerability of Antipsychotics in Treatment-Resistant Schizophrenia: A Network Meta-analysis. JAMA Psychiatry, 73(3), 199-210. doi:10.1001/jamapsychiatry.2015.2955

Seeman, P. (2014). Clozapine, a fast-off-D2 antipsychotic. ACS Chem Neurosci, 5(1), 24-29. doi:10.1021/ cn400189s

Seshamani, M. (2002). Is clozapine cost-effective? Unanswered issues. Eur J Health Econ, 3 Suppl 2, S104113. doi:10.1007/s10198-002-0115-8

Siafis, S., Tzachanis, D., Samara, M., \& Papazisis, G. (2018). Antipsychotic Drugs: From Receptor-binding Profiles to Metabolic Side Effects. Curr Neuropharmacol, 16(8), 1210-1223. doi:10.2174/157015 9x15666170630163616

Siskind, D., McCartney, L., Goldschlager, R., \& Kisely, S. (2016). Clozapine v. first- and second-generation antipsychotics in treatment-refractory schizophrenia: systematic review and meta-analysis. $\mathrm{Br} J$ Psychiatry, 209(5), 385-392. doi:10.1192/bjp.bp.115.177261

Siskind, D., Sidhu, A., Cross, J., Chua, Y. T., Myles, N., Cohen, D., \& Kisely, S. (2020). Systematic review and meta-analysis of rates of clozapine-associated myocarditis and cardiomyopathy. Aust $N Z J$ Psychiatry, 4867419898760. doi:10.1177/0004867419898760

Souza, J. S., Kayo, M., Tassell, I., Martins, C. B., \& Elkis, H. (2013). Efficacy of olanzapine in comparison with clozapine for treatment-resistant schizophrenia: evidence from a systematic review and metaanalyses. CNS Spectr, 18(2), 82-89. doi:10.1017/s1092852912000806

Stroup, T. S., Gerhard, T., Crystal, S., Huang, C., \& Olfson, M. (2014). Geographic and clinical variation in clozapine use in the United States. Psychiatr Serv, 65(2), 186-192. doi:10.1176/appi.ps.201300180

Stroup, T. S., Gerhard, T., Crystal, S., Huang, C., \& Olfson, M. (2016). Comparative Effectiveness of 
Clozapine and Standard Antipsychotic Treatment in Adults With Schizophrenia. Am J Psychiatry, 173(2), 166-173. doi:10.1176/appi.ajp.2015.15030332

Swildens, W., Termorshuizen, F., de Ridder, A., Smeets, H., \& Engelhard, I. M. (2016). Somatic Care with a Psychotic Disorder. Lower Somatic Health Care Utilization of Patients with a Psychotic Disorder Compared to Other Patient Groups and to Controls Without a Psychiatric Diagnosis. Adm Policy Ment Health, 43(5), 650-662. doi:10.1007/s10488-015-0679-0

Taipale, H., Mittendorfer-Rutz, E., Alexanderson, K., Majak, M., Mehtala, J., Hoti, F., . . Tiihonen, J. (2017). Antipsychotics and mortality in a nationwide cohort of 29,823 patients with schizophrenia. Schizophr Res. doi:10.1016/j.schres.2017.12.010

Taipale, H., Tanskanen, A., Mehtala, J., Vattulainen, P., Correll, C. U., \& Tiihonen, J. (2020). 20-year follow-up study of physical morbidity and mortality in relationship to antipsychotic treatment in a nationwide cohort of 62,250 patients with schizophrenia (FIN20). World Psychiatry, 19(1), 61-68. doi:10.1002/wps.20699

Tandon, R., Gaebel, W., Barch, D. M., Bustillo, J., Gur, R. E., Heckers, S., . . Carpenter, W. (2013). Definition and description of schizophrenia in the DSM-5. Schizophr Res, 150(1), 3-10. doi:10.1016/j.schres.2013.05.028

Termorshuizen, F., Wierdsma, A. I., Smeets, H. M., Visser, E., Drukker, M., Nijman, H., \& Sytema, S. (2013). Cause-specific mortality among patients with psychosis: disentangling the effects of age and illness duration. Psychosomatics, 54(6), 536-545. doi:10.1016/j.psym.2013.05.011

Thien, K., \& O'Donoghue, B. (2018). Delays and barriers to the commencement of clozapine in eligible people with a psychotic disorder: A literature review. Early Interv Psychiatry. doi:10.1111/eip.12683

Tiihonen, J., Lonnqvist, J., Wahlbeck, K., Klaukka, T., Niskanen, L., Tanskanen, A., \& Haukka, J. (2009). 11-year follow-up of mortality in patients with schizophrenia: a population-based cohort study (FIN11 study). Lancet, 374(9690), 620-627. doi:10.1016/s0140-6736(09)60742-x

Ucok, A., Cikrikcili, U., Karabulut, S., Salaj, A., Ozturk, M., Tabak, O., \& Durak, R. (2015). Delayed initiation of clozapine may be related to poor response in treatment-resistant schizophrenia. Int Clin Psychopharmacol, 30(5), 290-295. doi:10.1097/yic.0000000000000086

Veerman, S. R. T., Schulte, P. F. J., \& de Haan, L. (2017). Treatment for Negative Symptoms in Schizophrenia: A Comprehensive Review. Drugs, 77(13), 1423-1459. doi:10.1007/s40265-017-0789-y

Verdoux, H., Pambrun, E., Cortaredona, S., Coldefy, M., Le Neindre, C., Tournier, M., \& Verger, P. (2016). Geographical disparities in prescription practices of lithium and clozapine: a communitybased study. Acta Psychiatr Scand, 133(6), 470-480. doi:10.1111/acps.12554

Verdoux, H., Quiles, C., Bachmann, C. J., \& Siskind, D. (2018). Prescriber and institutional barriers and facilitators of clozapine use: A systematic review. Schizophr Res. doi:10.1016/j.schres.2018.05.046

Volavka, J., Czobor, P., Nolan, K., Sheitman, B., Lindenmayer, J. P., Citrome, L., . . Lieberman, J. A. (2004). Overt aggression and psychotic symptoms in patients with schizophrenia treated with clozapine, olanzapine, risperidone, or haloperidol. J Clin Psychopharmacol, 24(2), 225-228.

Wahlbeck, K., Cheine, M., \& Essali, M. A. (2000). Clozapine versus typical neuroleptic medication for schizophrenia. Cochrane Database Syst Rev(2), Cd000059. doi:10.1002/14651858.cd000059

Walker, A. M., Lanza, L. L., Arellano, F., \& Rothman, K. J. (1997). Mortality in current and former users 
of clozapine. Epidemiology, 8(6), 671-677.

West, S., Rowbotham, D., Xiong, G., \& Kenedi, C. (2017). Clozapine induced gastrointestinal hypomotility: A potentially life threatening adverse event. A review of the literature. Gen Hosp Psychiatry, 46, 32-37. doi:10.1016/j.genhosppsych.2017.02.004

Wheeler, A. J. (2008). Treatment pathway and patterns of clozapine prescribing for schizophrenia in New Zealand. Ann Pharmacother, 42(6), 852-860. doi:10.1345/aph.1K662

Wimberley, T., MacCabe, J. H., Laursen, T. M., Sorensen, H. J., Astrup, A., Horsdal, H. T., . . Stovring, H. (2017). Mortality and Self-Harm in Association With Clozapine in Treatment-Resistant Schizophrenia. Am J Psychiatry, 174(10), 990-998. doi:10.1176/appi.ajp.2017.16091097 
PART I

Clozapine prescription and monitoring 



\section{Abstract}

Purpose: To our knowledge, no study has examined in a structured way the extent of under-prescription of clozapine in ambulatory patients with Non-Affective Psychotic Disorder(NAPD). In the Netherlands, psychiatric care for such patients is provided by Flexible Assertive Community Treatment (FACT) teams and, by early intervention teams. In 20 FACT teams and 3 early intervention teams we assessed the proportion of patients who: use clozapine (type 1 patients), previously used this drug (type 2), have an unfulfilled indication for this drug, by type of indication (type 3), or were at least markedly psychotic, but had not yet received two adequate treatments with other antipsychotic drugs (type 4). We expected to find major differences between teams. To rule out that these differences are caused by differences in severity of psychopathology, we also calculated the proportions of patients who use clozapine given an indication at any time (number of type 1 patients divided by the sum of type 1, 2 and 3 patients).

Materials and methods: The nurse practitioner of each team identified the patients already on clozapine. Next, using a highly-structured decision tree, the nurse practitioner and psychiatrist assessed whether the remaining patients had an indication for this drug. Indications were treatment-resistant positive symptoms, tardive dyskinesia, aggression and suicidality. The severity of positive symptoms was determined using the Clinical Global Impression-Schizophrenia Scale

Results: In the participating FACT-teams 2,286 NAPD patients were assessed. The range among teams in proportions was: type $1: 8.8$ to $34.7 \%$ (mean: $23.0 \%$ ), type 2: 0 to $8.2 \%$ (mean: $3.5 \%$ ), type 3: 1.7 to $15.6 \%$ (mean: $6.9 \%$ ), type $4: 1.8$ to $16.3 \%$ (mean: $8.6 \%$ ). The range in proportions of patients using this drug given an indication was 49.0 to $90.9 \%$ (mean: $68.8 \%$ ). These figures were lower in early intervention teams.

Conclusions: The proportion of patients in FACT-teams who have an unfulfilled indication for clozapine is $6.9 \%$. There were considerable differences between teams with respect to this proportion. Almost a third of the outpatients had at any time an indication for clozapine. If one takes type 4 patients into account, this proportion may be higher.

Registration number: NTR5135 http://www.trialregister.nl/trialreg/index.asp

Keywords: clozapine; psychotic disorders; outpatient care; prescription rates 


\section{Introduction}

Despite the general idea that clozapine is underutilized, little research has been done into the extent of this problem. The main indication for this drug according to guidelines is treatment-resistant schizophrenia, the prevalence of which has been estimated at about 20-30\% (Kennedy, Altar, Taylor, Degtiar, \& Hornberger, 2014), but exact numbers are unknown. This uncertainty is not only caused by a scarcity of pertinent studies, but also by the absence (until recently) of consensus on criteria to define treatment-resistant schizophrenia (Howes et al., 2017). To illustrate this, Juarez-Reyes et al. (1995) found that the proportion in a population of outpatients was $12.9 \%$ with a stringent definition and $42.9 \%$ with a broad definition. A Danish register-based study among outpatients with a diagnosis of schizophrenia found a prevalence of $24.7 \%$ or $48.2 \%$ (Wimberley et al., 2016), depending on the definition of a proxy for treatment-resistant schizophrenia: 1) at least two different periods of antipsychotic use and one hospitalization within 18 months and 2) patients treated with polypharmacy for at least 90 days. No information on adherence and symptom severity was available, which precludes an exact assessment of treatment-resistant schizophrenia.

Furthermore, there are also other indications for clozapine, as it has been found to decrease tardive dyskinesia (Hazari, Kate, \& Grover, 2013), acute extrapyramidal symptoms (, i.e. parkinsonism, acute dystonia and akathisia) (Leucht et al., 2013), aggression (Frogley, Taylor, Dickens, \& Picchioni, 2012; Krakowski, Czobor, Citrome, Bark, \& Cooper, 2006; Volavka et al., 2004), suicidality (Hennen \& Baldessarini, 2005; Meltzer et al., 2003) and substance abuse (Kelly, Daley, \& Douaihy, 2012). The prevalence of these other indications is even more uncertain.

Studies of clozapine prescription rates show large international differences (Nielsen, Roge, Schjerning, Sorensen, \& Taylor, 2012; Stroup, Gerhard, Crystal, Huang, \& Olfson, 2014; Verdoux et al., 2016). In the Netherlands, the proportion of patients with Non-Affective Psychotic Disorder (NAPD) using this drug is unknown. The vast majority of them is treated on an ambulatory basis by Flexible Assertive Community Treatment (FACT) teams and in some regions also by early intervention teams. FACT-teams take care of patients with a severe mental illness and are called "flexible" because they intensify treatment when the patient is in a crisis, with the aim to prevent hospitalization (van Veldhuizen, 2007). These teams are responsible for a certain area and treat approximately 200 outpatients, most of whom with NAPD. Some institutes deploy specialized early intervention teams to treat patients in the first years after onset of psychosis. These teams work in the same way as the FACT-teams, but they spend more time on diagnosing patients and providing psychoeducation. Their caseloads may be smaller and the patients are younger. After a maximum of five year, treatment will be 
continued by a FACT-team. In general, in every FACT or early intervention team there is a psychiatrist, but only part of the teams have a nurse practitioner associated with it.

Summarizing, little is known about the magnitude of underprescription of clozapine in outpatients with NAPD in the Netherlands. We set out to examine in a structured way rates of prescription and underprescription in FACT teams and in early intervention teams. We developed a decision tree with criteria for an indication for clozapine. Our definition of treatment resistance differs only slightly from the consensus guideline that was published shortly after we collected our data (Nielsen, Dahm, Lublin, \& Taylor, 2010). Since in our experience there are major regional and personal differences in adherence to guidelines regarding clozapine prescription, we expected considerable differences between teams in proportions of patients using this drug and patients with an unfulfilled indication. In order to exclude the possibility that these differences are solely caused by differences in the severity of psychopathology, we calculated for each team the proportion of patients who use clozapine given an indication at any time.

The aims of this study were to determine prescription rates and the extent of underprescription of clozapine in outpatients with NAPD. We therefore assessed the proportions of patients who (i) currently use clozapine (type 1 patients); (ii) had used this drug and subsequently discontinued it (type 2 patients); (iii) have an indication for this drug but have never used it, by type of indication (type 3 patients); (iv) were at least markedly psychotic, but had not yet received two adequate treatments with antipsychotic drugs (type 4 patients); (v) currently use clozapine among patients with an indication for this drug at any time (number of type 1 patients divided by the sum of type 1 , type 2 and type 3 patients).

\section{Materials and methods}

\section{Study design and setting}

This study reports the results of baseline measurements preceding a randomized controlled trial to assess the safety of the deployment of nurse practitioners to start patients on clozapine. Therefore, in this study, only teams with a nurse practitioner associated with it were included. Twenty FACT teams and three early intervention teams, from four Dutch psychiatric institutes, participated. Each institute deploys several teams, housed in the same building or at miles distance from each other. In all participating teams, the psychiatrist was responsible for the prescription of antipsychotic drugs. Data was collected from July 2015 to May 2016. 


\section{Measures}

According to Dutch guidelines ("Dutch Clozapine Collaboration Group. Guideline for the use of clozapine [English version]. ," 2009; Van Alphen, 2012), clozapine is indicated for patients with a diagnosis of schizophrenia or schizoaffective disorder, who suffer from 1) treatment-resistant positive or negative symptoms, 2) severe aggressive behavior, 3) persistent suicidal behavior, 4) tardive dyskinesia, 5) treatment-resistant acute extrapyramidal symptoms, and 6) alcohol or drug abuse (Van Alphen, 2012). However, since the current evidence to support the use of clozapine for treatmentresistant negative symptoms or substance abuse is insufficient, this study did not regard these features as indications for clozapine.

In order to structure the assessment of an indication for clozapine, we developed a decision tree (see Appendix). Positive symptoms were scored using the Clinical Global Impression-Schizophrenia Scale (CGI-SCH), a simple instrument, appropriate for use in observational studies (Haro et al., 2003). The researchers who developed this instrument, reported that the correlation coefficient between the CGI-SCH for positive symptoms and the PANSS score was 0.86 and the interrater reliability was high (intraclass correlation coefficient, ICC $=0.82$ ). Possible scores for positive symptoms are "normal, not ill" (1), "minimally ill" (2), "mildly ill" (3), "moderately ill" (4), "markedly ill (5), "severely ill" (6) and "among the most severely ill" (7) (20) (see Appendix for a more detailed description of the scores). We defined treatment-resistance of positive symptoms as the persistence of at least markedly severe positive symptoms (score 5 or higher), despite adequate treatment. Adequate treatment was defined as having used two different antipsychotics, of which at least one second generation antipsychotic, during at least 4 weeks in an adequate dosage. A list of adequate dosages of antipsychotic medication (see Appendix) was constructed using studies on comparable dosages of antipsychotics (Andreasen, Pressler, Nopoulos, Miller, \& Ho, 2010; Lieberman et al., 2005; Loebel et al., 2015), information from the World Health Organization on Defined Daily Dosages ("Dutch Clozapine Collaboration Group. Guideline for the use of clozapine [English version]. ," 2009; "WHO ATC/DDD index N05A Antipsychotics,") and Dutch guidelines (Van Alphen, 2012). Three other indications for clozapine (markedly severe tardive dyskinesia, aggressive behavior or suicidality, all persisting during the use of two other antipsychotics) were also elaborated in the decision tree (Frogley et al., 2012; Hazari et al., 2013; Hennen \& Baldessarini, 2005; Krakowski et al., 2006; Leucht et al., 2013; Meltzer et al., 2003; Volavka et al., 2004).

\section{Procedures}

In June 2015, the psychiatrists and nurse practitioners of each team followed a training in the assessment of an indication for clozapine, during which the decision tree was introduced. After the training, the nurse practitioner identified all patients with NAPD by 
checking the DSM-IV codes, schizophrenia, schizophreniform disorder, schizoaffective disorder or psychotic disorder not otherwise specified. The latter diagnosis was included because some Dutch psychiatrists are reluctant to use the word schizophrenia. In part of the teams, the controversy surrounding the concept of schizophrenia seems to have led to an increase of the diagnosis psychotic disorder not otherwise specified, and a lower use of schizophrenia as diagnostic label.

Patients with delusional disorder were not included, because clozapine has not shown to be effective for them. Those diagnosed with a brief psychotic disorder were also excluded because clozapine is not indicated for patients with this diagnosis.

The nurse practitioner of each team, assisted by the first author, reviewed the files of all the patients treated by that team. They identified the patients who were already on clozapine or had used this drug and had discontinued it. We assumed that all of these patients had an appropriate indication for this drug. Next, using the decision tree, the nurse practitioner, again assisted by the first author, assessed the remaining patients for clozapine indications, regardless of the feasibility of a trial with clozapine. These patients were divided into 3 groups: a) no indication, b) indication, and c) questionable indication (for example a score of 4 "moderate" on the CGI-SCH or uncertainty about other indications or previous treatment with antipsychotics) Subsequently, they discussed the cases from the latter two categories with the responsible psychiatrist and tried to reach consensus about the indication for a trial with clozapine. In case of discordance, the opinion of the psychiatrist was decisive.

\section{Statistical analysis}

Descriptive statistics were used to summarize demographic and clinical characteristics. $\chi^{2}$-tests were used to compare teams on all four types of patients.

After a Bonferroni correction for multiple testing, a two-tailed $p$-value of $<0.008$ was considered statistically significant for all tests.

\section{Results}

\section{Demographic and clinical characteristics}

In the FACT-teams, there were 2286 patients with NAPD and in the early intervention teams 302 patients. The characteristics of these patients are presented in Table 1. 
Table 1. Demographic and clinical features of 2588 patients with Non-Affective Psychotic Disorder, treated by 20 Functional Assertive Community Treatment (FACT) teams and three Early Intervention teams in the Netherlands, 2016.

\begin{tabular}{lccc}
\hline Characteristic & Total & $\begin{array}{c}\text { Clozapine, } \\
\text { current } \\
\text { users } \\
\text { (Type 1) }\end{array}$ & $\begin{array}{c}\text { Other } \\
\text { patients }\end{array}$ \\
\hline FACT teams & \multicolumn{2}{c}{$\begin{array}{c}\text { n=2286 } \\
\text { Mean (SD) }\end{array}$} & $\begin{array}{c}\text { Mean (SD) } \\
\text { Mean (SD) }\end{array}$ \\
\hline Age (years) & $46.5(11.6)$ & $46.5(11.6)$ & $47.0(11.9)$ \\
Sex & $\%$ & $\%$ & $\%$ \\
$\quad$ Male & 65.9 & 68.1 & 64.4 \\
DSM-IV diagnosis & & & \\
Schizophrenia (including schizophreniform disorder) & 65.0 & 82.5 & 58.9 \\
Schizoaffective disorder & 15.2 & 12.9 & 15.8 \\
Psychotic disorder not otherwise specified & 19.8 & 4.6 & 25.3 \\
\hline Early intervention teams & $\mathrm{n}=302$ & $\mathrm{n}=34$ & $\mathrm{n}=268$ \\
& Mean (SD) & Mean (SD) & Mean (SD) \\
\hline Age & $33.8(10.1)$ & $29.6(5.9)$ & $34.2(10.4)$ \\
Sex & $\%$ & $\%$ & $\%$ \\
Male & 68.9 & 79.4 & 67.7 \\
DSM-IV diagnosis & & & \\
Schizophrenia (including schizophreniform disorder) & 47.3 & 67.7 & 44.5 \\
Schizoaffective disorder & 6.0 & 8.8 & 5.9 \\
Psychotic disorder not otherwise specified & 46.7 & 23.5 & 49.6 \\
\hline & & & \\
\hline
\end{tabular}

\section{Use of clozapine}

The overall actual clozapine prescription rate among FACT- and early interventionteams (type 1 patients) was $21.6 \%$. The overall proportion of patients with an indication for clozapine at any time type 1, type 2 and type 3) was $33.4 \%$ in FACT-teams and $18.2 \%$ in early intervention-teams (overall: $31.6 \%$ ). Of these patients $68.3 \%$ was using clozapine (FACT-teams: 68.8\% and early intervention-teams: 61.8\%) also with a high variability between teams, see Table 2 .

The proportions of type 1 , type 2 , type 3 and type 4 patients and the ranges between teams are shown in Table 2 , by type of team. We found a significant variability between teams with regard to the proportions of all 4 types of patients (see figure 1). The overall rate of underprescription (type 3 patients) was 6.6\%. In $94.8 \%$ of these patients treatment-resistant positive symptoms were the reason for the clozapine indication (see for indications and combinations of indications Table 3). Only 5.2\% of patients had other indications without treatment-resistant positive symptoms. 
Table 2. Mean proportion and range of type 1, type 2, type 3 and type 4 patients and results of $\chi^{2}$ tests to compare teams, in 20 Flexible Assertive Community Treatment (FACT)-teams and 3 Early intervention teams in the Netherlands, 2016.

\begin{tabular}{|c|c|c|c|c|c|}
\hline FACT-teams n=2286 & $\%$ & Range & $\chi^{2}$ & df & $\bar{p}$ \\
\hline Type 1 (users) & 23.0 & $8.8-34.7$ & 53.6 & 19 & $<.001^{*}$ \\
\hline Type 2 (former users) & 3.5 & $0-\quad 8.2$ & 26.75 & 19 & .110 \\
\hline Type 3 (unfulfilled indication) & 6.9 & $1.7-15.6$ & 55.97 & 19 & $<.001^{*}$ \\
\hline Type 4 (as yet insufficiently treated) & 8.6 & $1.8-16.3$ & 52.29 & 19 & $<.001^{*}$ \\
\hline Total of type 1, 2 and 3 & 33.4 & $17.6-47.6$ & 66.97 & 19 & $<.001^{*}$ \\
\hline Users among type 1,2 and 3 & 68.8 & $49.0-90.9$ & 46.64 & 19 & $<.001^{*}$ \\
\hline Early intervention teams & $\%$ & Range & $\chi^{2}$ & df & $\mathbf{p}$ \\
\hline Type 1 (users) & 11.3 & $8.5-14.1$ & 1.35 & 2 & .509 \\
\hline Type 2 (former users) & 2.3 & $1.1-\quad 3.6$ & 1.88 & 2 & .391 \\
\hline Type 3 (unfulfilled indication) & 4.6 & $0-\quad 9.4$ & 13.14 & 2 & $.001^{*}$ \\
\hline Type 4 (as yet insufficiently treated) & 11.6 & $7.0-16.3$ & 3.51 & 2 & .171 \\
\hline Total of type 1, 2 and 3 & 18.2 & $11.3-23.7$ & 5.70 & 2 & .058 \\
\hline Users among type 1, 2 and 3 & 61.8 & $45.5-92.9$ & 10.04 & 2 & .007 \\
\hline
\end{tabular}

$*$ A p-value of $<.008$ was considered statistically significant

Table 3. Type of indication for clozapine among 172 patients with Non-Affective Psychotic Disorder, with an unfulfilled indication, from 20 Flexible Assertive Community Treatment (FACT)-teams and 3 early intervention teams in the Netherlands, 2016.

\begin{tabular}{lcc}
\hline Single indication $\mathbf{N}=\mathbf{1 3 4}$ & $\mathbf{N}$ & $\mathbf{\%}$ \\
\hline Treatment-resistant positive symptoms & 125 & 72.7 \\
Aggression & 1 & 0.6 \\
Suicidality & 3 & 1.7 \\
Acute extrapyramidal symptoms & 3 & 1.7 \\
Tardive dyskinesia & 2 & 1.2 \\
\hline Multiple indications $\mathbf{N}=\mathbf{3 8}$ & & \\
\hline Treatment-resistant positive symptoms & & \\
And Aggression & 24 & 14.0 \\
And Suicidality & 8 & 4.7 \\
And Suicidality + Aggression & 1 & 0.6 \\
And Extrapyramidal symptoms & 2 & 1.2 \\
And Tardive dyskinesia & 1 & 0.6 \\
And Extrapyramidal symptoms + tardive dyskinesia & 2 & 1.2 \\
\hline
\end{tabular}




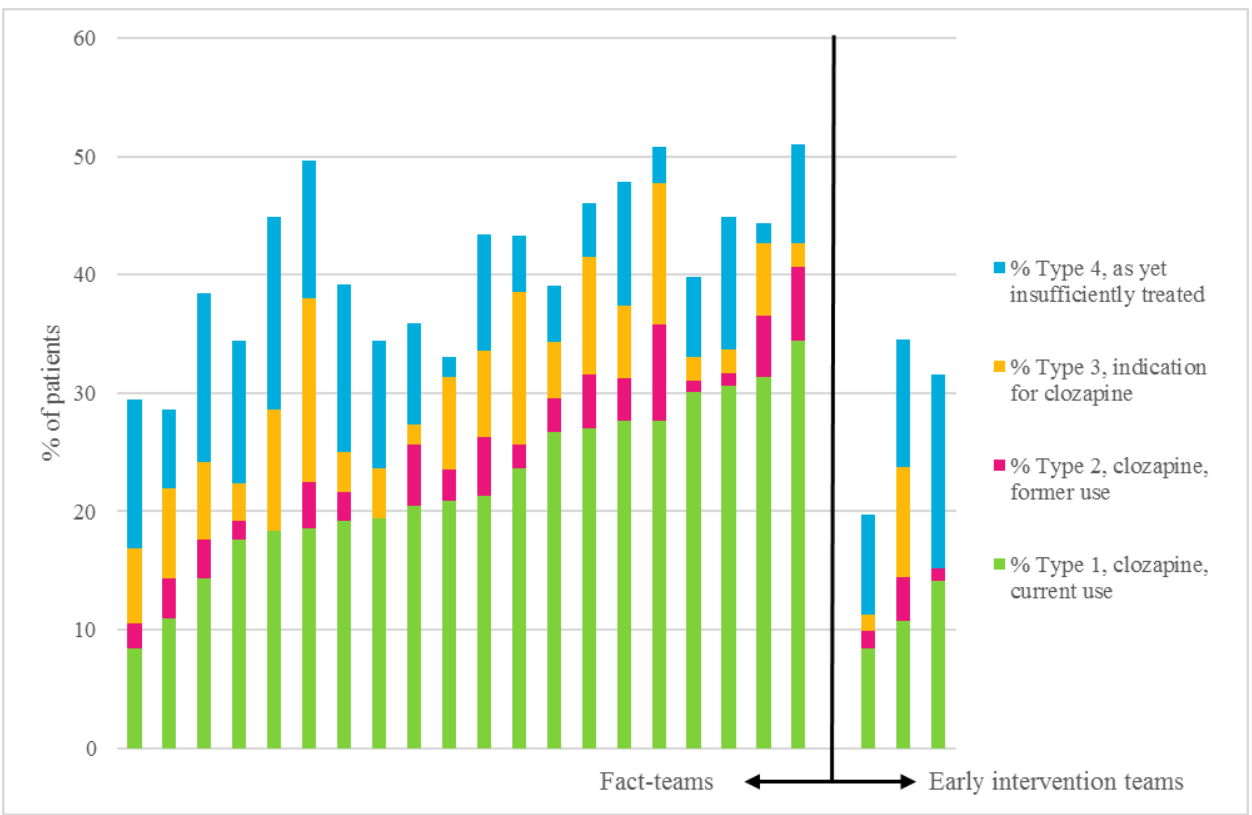

Figure 1. Per team: proportions of current users of clozapine, of previous users, of those with an indication for this drug, and of those as yet insufficiently treated to have a diagnosis of treatment-resistance. The first 20 bars are the FACT-teams, and the last three bars are the early intervention teams.

\section{Discussion}

\section{Main findings}

The prescription rate of clozapine in FACT- and early intervention-teams was 21.6\% (type 1 patients), and the rate of underprescription was $6.6 \%$ (type 3). However, the latter proportion is probably higher, because a part of the as yet insufficiently treated patients (type 4) may turn out to have an indication for this drug too. The differences between teams in prescribing and underprescribing clozapine, were very large and statistically significant.

\section{Interpretation}

The proportion of outpatients with NAPD on clozapine in this study is higher than those reported by other European studies in outpatients. A national database study in Denmark found that $10.1 \%$ of the patients with a diagnosis of schizophrenia was using clozapine (Nielsen et al., 2012).. In a study in France, only 1.2\% of the users of antipsychotics was using clozapine. However, $6.6 \%$ of the total population was using antipsychotics, so the clozapine prescription rate among patients with NAPD may be much higher (Verdoux et al., 2016). Although different study designs may contribute to these differences, a large international database study (Bachmann et al., 2017) also showed that prescription rates 
in the Netherlands are higher than in most other countries. A national clozapine expertise center, founded in 2004, may have contributed to this (Bogers, Schulte, Van Dijk, Bakker, \& Cohen, 2016). An audit in the UK, with a comparable real-world design found similar rates of $23.7 \%$ (Patel et al., 2014). However, Patel et al. only included patients that were under care for at least 12 months, which may have led to somewhat higher clozapine prescription rates. They found a proportion of $21.2 \%$ of patients who were not or partially in remission (no definition given) and were not prescribed clozapine. Sixty-one percent of these patients had already received two adequate trials of antipsychotics, suggesting that $12.9 \%$ was having an unfulfilled indication for clozapine. However, drug adherence had been investigated for only $85 \%$ of them.

We found considerable difference in clozapine prescription rates between teams which may partly be caused by differences in the severity of psychopathology. However, we also found significant differences in prescription rates after restricting the analysis to patients with an indication for this drug. It is unlikely therefore, that the variability between teams is entirely explained by differences in severity of psychopathology. Other explanatory variables are local norms and traditions (Howes et al., 2012; Olfson, Gerhard, Crystal, \& Stroup, 2016; Verdoux et al., 2016) or specific clinician-related factors, such as their workload, knowledge and preferences (Patel, 2012). The more the time of the psychiatrist is restricted, the more difficult it may become to supervise the weekly blood drawings and monitor adequately for potentially lethal side-effects. Consequently, extra staff, such as the deployment of a nurse practitioner, may help in preventing needless delay in clozapine initiation (Gee, Vergunst, Howes, \& Taylor, 2014).

\section{Strengths and limitations}

A strength of this study is the large number of patients from both FACT- and early intervention-teams from four different institutes. Additionally, we were able to determine the exact proportion on clozapine and the decision tree allowed for a standardized method to assess indications for clozapine. However, several limitations require comment. First, the teams of this study belonged to institutes that had agreed to participate in a trial on the safety of the deployment of nurse practitioners to start patients on clozapine. Consequently, the non-random selection of teams diminishes the generalizability of the results. Second, the quality of the information obtained from electronic files was not optimal in all cases. Some diagnoses may have been inaccurate and some information on antipsychotics was lost during the transition from paper file to electronic file, about 10 years ago. Information on the presence of tardive dyskinesia was often lacking and was almost solely obtained verbally from the responsible nurse practitioner or psychiatrist. Third, the rating of the severity of the positive symptoms may not always have been perfectly valid. However, since there were only 2 cases of discordance on the indication for clozapine, the decisive role of the psychiatrist barely influenced the results. Finally, 
our definition of treatment-resistance differed somewhat from that in recently published guidelines (Howes et al., 2017), in that it was based on a higher score for the severity of positive symptoms (marked instead of moderate), on a adherence of $90 \%$ of prescribed antipsychotics taken (instead of $80 \%$ ), but on a shorter duration of adequate treatment ( 4 instead of 6 weeks) and on a slightly lower minimum dosage of antipsychotic drug, see Appendix. Consequently, a substantial over- or underestimation of the number of indications for clozapine is highly unlikely.

\section{Conclusion}

In conclusion, about a third of the Dutch outpatients with NAPD is indicated for the use of clozapine and more than two-thirds of them are using it. By international standards, the clozapine prescription rates in Dutch ambulatory care are high, but the differences between teams are considerable. Research into reasons for this variability is urgently needed to develop targeted interventions.

\section{Acknowledgements}

The authors would like to thank all participating psychiatrists and nurse practitioners for helping to provide the data necessary for this study.

\section{Funding}

This research did not receive any specific grant from funding agencies in the public, commercial or not-for-profit sectors.

\section{Authors' contribution}

Author JS, PS and YZ designed the study and wrote the protocol. Author FT and YZ undertook the statistical analysis, and author $\mathrm{YZ}$ wrote the first draft of the manuscript. All authors contributed to and have approved the final manuscript. 


\section{Compliance with ethical standards}

This study complies with the ethical rules for human experimentation as stated in the Declaration of Helsinki and participation was voluntary. The study was approved by the Medical Ethics Committee of the RTPO (Regionale Toetsingscommissie Patiëntgebonden Onderzoek), Leeuwarden, the Netherlands.

\section{Conflict of interest}

The authors declare that they have no conflicts of interests. 


\section{References}

Andreasen, N. C., Pressler, M., Nopoulos, P., Miller, D., \& Ho, B. C. (2010). Antipsychotic dose equivalents and dose-years: a standardized method for comparing exposure to different drugs. Biol Psychiatry, 67(3), 255-262. doi:10.1016/j.biopsych.2009.08.040

Bachmann, C. J., Aagaard, L., Bernardo, M., Brandt, L., Cartabia, M., Clavenna, A., . . Taylor, D. (2017). International trends in clozapine use: a study in 17 countries. Acta Psychiatr Scand, 136(1), 37-51. doi:10.1111/acps.12742

Bogers, J. P., Schulte, P. F., Van Dijk, D., Bakker, B., \& Cohen, D. (2016). Clozapine Underutilization in the Treatment of Schizophrenia: How Can Clozapine Prescription Rates Be Improved? J Clin Psychopharmacol, 36(2), 109-111. doi:10.1097/jcp.0000000000000478

Dutch Clozapine Collaboration Group. Guideline for the use of clozapine [English version]. . (2009). Retrieved from http://clozapinepluswerkgroep.nl/pdf/SKMBT_C45110040808460.pdf.

Frogley, C., Taylor, D., Dickens, G., \& Picchioni, M. (2012). A systematic review of the evidence of clozapine's anti-aggressive effects. Int J Neuropsychopharmacol, 15(9), 1351-1371. doi:10.1017/ s146114571100201x

Gee, S., Vergunst, F., Howes, O., \& Taylor, D. (2014). Practitioner attitudes to clozapine initiation. Acta Psychiatr Scand, 130(1), 16-24. doi:10.1111/acps.12193

Haro, J. M., Kamath, S. A., Ochoa, S., Novick, D., Rele, K., Fargas, A., . . Jones, P. B. (2003). The Clinical Global Impression-Schizophrenia scale: a simple instrument to measure the diversity of symptoms present in schizophrenia. Acta Psychiatr Scand Suppl(416), 16-23.

Hazari, N., Kate, N., \& Grover, S. (2013). Clozapine and tardive movement disorders: a review. Asian J Psychiatr, 6(6), 439-451. doi:10.1016/j.ajp.2013.08.067

Hennen, J., \& Baldessarini, R. J. (2005). Suicidal risk during treatment with clozapine: a meta-analysis. Schizophr Res, 73(2-3), 139-145. doi:10.1016/j.schres.2004.05.015

Howes, O. D., McCutcheon, R., Agid, O., de Bartolomeis, A., van Beveren, N. J., Birnbaum, M. L., . . . Correll, C. U. (2017). Treatment-Resistant Schizophrenia: Treatment Response and Resistance in Psychosis (TRRIP) Working Group Consensus Guidelines on Diagnosis and Terminology. Am J Psychiatry, 174(3), 216-229. doi:10.1176/appi.ajp.2016.16050503

Howes, O. D., Vergunst, F., Gee, S., McGuire, P., Kapur, S., \& Taylor, D. (2012). Adherence to treatment guidelines in clinical practice: study of antipsychotic treatment prior to clozapine initiation. $\mathrm{Br} J$ Psychiatry, 201(6), 481-485. doi:10.1192/bjp.bp.111.105833

Juarez-Reyes, M. G., Shumway, M., Battle, C., Bacchetti, P., Hansen, M. S., \& Hargreaves, W. A. (1995). Effects of stringent criteria on eligibility for clozapine among public mental health clients. Psychiatr Serv, 46(8), 801-806. doi:10.1176/ps.46.8.801

Kelly, T. M., Daley, D. C., \& Douaihy, A. B. (2012). Treatment of substance abusing patients with comorbid psychiatric disorders. Addict Behav, 37(1), 11-24. doi:10.1016/j.addbeh.2011.09.010

Kennedy, J. L., Altar, C. A., Taylor, D. L., Degtiar, I., \& Hornberger, J. C. (2014). The social and economic burden of treatment-resistant schizophrenia: a systematic literature review. Int Clin Psychopharmacol, 29(2), 63-76. doi:10.1097/YIC.0b013e32836508e6 
Krakowski, M. I., Czobor, P., Citrome, L., Bark, N., \& Cooper, T. B. (2006). Atypical antipsychotic agents in the treatment of violent patients with schizophrenia and schizoaffective disorder. Arch Gen Psychiatry, 63(6), 622-629. doi:10.1001/archpsyc.63.6.622

Leucht, S., Cipriani, A., Spineli, L., Mavridis, D., Orey, D., Richter, F., . . Davis, J. M. (2013). Comparative efficacy and tolerability of 15 antipsychotic drugs in schizophrenia: a multiple-treatments metaanalysis. Lancet, 382(9896), 951-962. doi:10.1016/s0140-6736(13)60733-3

Lieberman, J. A., Stroup, T. S., McEvoy, J. P., Swartz, M. S., Rosenheck, R. A., Perkins, D. O., . . Hsiao, J. K. (2005). Effectiveness of antipsychotic drugs in patients with chronic schizophrenia. $N$ Engl J Med, 353(12), 1209-1223. doi:10.1056/NEJMoa051688

Loebel, A., Cucchiaro, J., Silva, R., Mao, Y., Xu, J., Pikalov, A., \& Marder, S. R. (2015). Efficacy of lurasidone across five symptom dimensions of schizophrenia: pooled analysis of short-term, placebo-controlled studies. Eur Psychiatry, 30(1), 26-31. doi:10.1016/j.eurpsy.2014.08.001

Meltzer, H. Y., Alphs, L., Green, A. I., Altamura, A. C., Anand, R., Bertoldi, A., . . Potkin, S. (2003). Clozapine treatment for suicidality in schizophrenia: International Suicide Prevention Trial (InterSePT). Arch Gen Psychiatry, 60(1), 82-91.

Nielsen, J., Dahm, M., Lublin, H., \& Taylor, D. (2010). Psychiatrists' attitude towards and knowledge of clozapine treatment. J Psychopharmacol, 24(7), 965-971. doi:10.1177/0269881108100320

Nielsen, J., Roge, R., Schjerning, O., Sorensen, H. J., \& Taylor, D. (2012). Geographical and temporal variations in clozapine prescription for schizophrenia. Eur Neuropsychopharmacol, 22(11), 818-824. doi:10.1016/j.euroneuro.2012.03.003

Olfson, M., Gerhard, T., Crystal, S., \& Stroup, T. S. (2016). Clozapine for Schizophrenia: State Variation in Evidence-Based Practice. Psychiatr Serv, 67(2), 152. doi:10.1176/appi.ps.201500324

Patel, M. X. (2012). Clinician hesitation prior to clozapine initiation: is it justifiable? Br J Psychiatry, 201(6), 425-427. doi:10.1192/bjp.bp.112.114777

Patel, M. X., Bishara, D., Jayakumar, S., Zalewska, K., Shiers, D., Crawford, M. J., \& Cooper, S. J. (2014). Quality of prescribing for schizophrenia: evidence from a national audit in England and Wales. Eur Neuropsychopharmacol, 24(4), 499-509. doi:10.1016/j.euroneuro.2014.01.014

Stroup, T. S., Gerhard, T., Crystal, S., Huang, C., \& Olfson, M. (2014). Geographic and clinical variation in clozapine use in the United States. Psychiatr Serv, 65(2), 186-192. doi:10.1176/appi.ps.201300180

Van Alphen, C. A., M. Blanke, C. (2012). Multidisciplinaire richtlijn schizofrenie (Multidisciplinairy Guidelines for Schizophrenia). In (pp. 112). Utrecht: Landelijke stuurgroep Multidisciplinaire Richtlijnontwikkeling and Trimbos Instituut [Netherlands Institute of Mental health and Addiction].

van Veldhuizen, J. R. (2007). FACT: a Dutch version of ACT. Community Ment Health J, 43(4), 421-433. doi:10.1007/s10597-007-9089-4

Verdoux, H., Pambrun, E., Cortaredona, S., Coldefy, M., Le Neindre, C., Tournier, M., \& Verger, P. (2016). Geographical disparities in prescription practices of lithium and clozapine: a communitybased study. Acta Psychiatr Scand, 133(6), 470-480. doi:10.1111/acps.12554

Volavka, J., Czobor, P., Nolan, K., Sheitman, B., Lindenmayer, J. P., Citrome, L., . . Lieberman, J. A. (2004). Overt aggression and psychotic symptoms in patients with schizophrenia treated with 
clozapine, olanzapine, risperidone, or haloperidol. J Clin Psychopharmacol, 24(2), 225-228.

WHO ATC/DDD index N05A Antipsychotics. (2017-12-20). Retrieved from (5) http://www.whocc.no/ atc_ddd_index/?code=N05A

Wimberley, T., Stovring, H., Sorensen, H. J., Horsdal, H. T., MacCabe, J. H., \& Gasse, C. (2016). Predictors of treatment resistance in patients with schizophrenia: a population-based cohort study. Lancet Psychiatry, 3(4), 358-366. doi:10.1016/s2215-0366(15)00575-1 


\section{Appendix}

\section{Adequate dosages, description of the scores on the Clinical Global} Impression-Schizophrenia Scale (CGI-SCH), and the decision tree. Adequate dosage, oral medication

\begin{tabular}{lr}
\hline Drug & Adequate dosage \\
\hline Aripiprazole & $15 \mathrm{mg} / \mathrm{d}(2)$ \\
Bromperidol & $4 \mathrm{mg} / \mathrm{d}$ \\
Flupentixol & $4 \mathrm{mg} / \mathrm{d}$ \\
Haloperidol & $4 \mathrm{mg} / \mathrm{d}(3)$ \\
Lurasidone & $40 \mathrm{mg} / \mathrm{d} \mathrm{(7)}$ \\
Olanzapine & $15 \mathrm{mg} / \mathrm{d}(2)$ \\
Paliperidone & $6 \mathrm{mg} / \mathrm{d}(5)$ \\
Penfluridol & $40 \mathrm{mg} / \mathrm{wk}(5)$ \\
Perphenazine & $16 \mathrm{mg} / \mathrm{d}(2)$ \\
Pimozide & $4 \mathrm{mg} / \mathrm{d}(5)$ \\
Quetiapine & $400 \mathrm{mg} / \mathrm{d}(2)$ \\
Risperidone & $3 \mathrm{mg} / \mathrm{d}(2)$ \\
Sertindole & $12-20 \mathrm{md} / \mathrm{d}(6)$ \\
Sulpiride & $800 \mathrm{mg} / \mathrm{d}(5)$ \\
Zuclopenthixol & $16 \mathrm{mg} / \mathrm{d}(4)$ \\
\hline
\end{tabular}

Adequate dosage, long lasting injectables

\begin{tabular}{lr}
\hline Drug & Adequate dosage \\
\hline Aripiprazole & $400 \mathrm{mg} / 4 \mathrm{wk}(6)$ \\
Bromperidol & $100 \mathrm{mg} / 4 \mathrm{wk}$ \\
Fluphenazine & $50 \mathrm{mg} / 4 \mathrm{wk}(1)$ \\
Flupentixol & $40 \mathrm{mg} / 2 \mathrm{wk}(1)$ \\
Fluspirilene & $4 \mathrm{mg} / \mathrm{wk}(1)$ \\
Haloperidol & $100 \mathrm{mg} / 4 \mathrm{wk}(1)$ \\
Olanzapine & $210 \mathrm{mg} / 2 \mathrm{wk}(6)$ \\
Paliperidone & $75 \mathrm{mg} / 4 \mathrm{wk}(6)$ \\
Risperidone & $37,5 \mathrm{mg} / 2 \mathrm{wk}(1)$ \\
Zuclopenthixol & $225 \mathrm{mg} / 3 \mathrm{wk}(1)$
\end{tabular}

(1) Moleman P, Birkenhäger T. Praktische Psychofarmacologie 2009. Houten: Bohn Stafleu Van Loghum.

(2) Lieberman J et al. New England Journal of Medicine 2005;353(12):1209-1223.

(3) Andreasen $\mathrm{N}$ et al. Biological psychiatry 2010;67(3):255-262.

(4) Van Alphen C et al. Multidisciplinaire richtlijn schizofrenie 2012. Utrecht: De Tijdstroom.

(5) http://www.whocc.no/atc_ddd_index/?code=N05A

(6) Van Loenen A. Farmacotherapeutisch Kompas 2003. Amstelveen.

(7) Loebel A et al. European Psychiatry 2015;30(1):26-31.

N.B. If the dosage was lower or the period shorter, due to untreatable EPS, this counts as adequate treatment. 


\section{Description of the scores on the Clinical Global Impression-Schizophrenia Scale (CGI-SCH).}

$1=$ Normal-not at all ill, symptoms of disorder not present past seven days.

2=Borderline mentally ill-subtle or suspected pathology.

3=Mildly ill-clearly established symptoms with minimal, if any, distress or difficulty in social and occupational function.

4=Moderately ill-overt symptoms causing noticeable, but modest, functional impairment or distress, symptom level may warrant medication.

5=Markedly ill-intrusive symptoms that distinctly impair social/occupational function or cause intrusive levels of distress.

$6=$ Severely ill-disruptive pathology, behavior and function are frequently influenced by symptoms, may require assistance from others.

7=Among the most extremely ill patients-pathology drastically interferes in many life functions; may be hospitalized. 


\section{Decision tree}

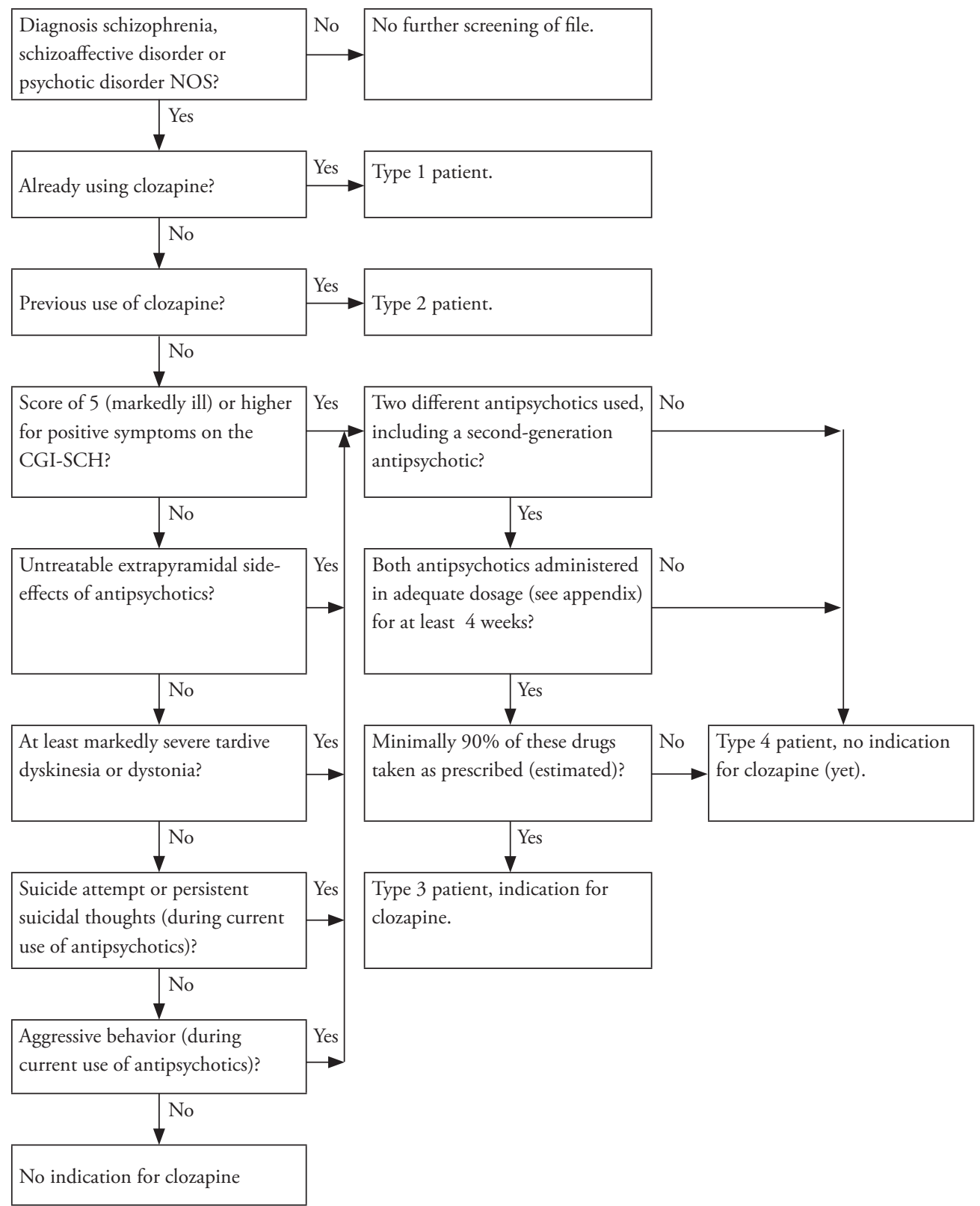





\section{Abstract}

Purpose: To test whether: (1) psychiatrists will prescribe clozapine more often if they can delegate the monitoring tasks to an advanced nurse practitioner (ANP), (2) clozapine monitoring by an ANP is at least as safe as monitoring by a psychiatrist.

Methods: Patients from 23 Dutch outpatient teams were assessed for an indication for clozapine. ANPs affiliated to these teams were randomized to Condition A: clozapine monitoring by an ANP, or Condition B: monitoring by the psychiatrist. The safety of monitoring was evaluated by determining whether the weekly neutrophil measurements were performed. Staff and patients were blinded regarding the first hypothesis.

Results: Of the 173 patients with an indication for clozapine at baseline, only 7 in Condition A and 4 in Condition B were prescribed clozapine (Odds Ratio=2.24, 95\% CI $0.61-8.21 ; \mathrm{p}=.225)$. These low figures affected the power of this study. When we considered all patients who started with clozapine over the 15-month period ( $\mathrm{N}=49$ ), the Odds Ratio was 1.90 (95\% CI 0.93-3.87; $\mathrm{p}=.078$ ). With regard to the safety of the monitoring of the latter group of patients, $71.2 \%$ of the required neutrophil measurements were performed in condition $A$ and $67.3 \%$ in condition $\mathrm{B}(\mathrm{OR}=0.98 ; \mathrm{CI}=0.16-3.04 ; \mathrm{p}=.98)$.

Conclusions: Identifying patients with an indication for clozapine does not automatically lead to improved prescription rates, even when an ANP is available for the monitoring. Clozapine-monitoring performed by an ANP seemed as safe as that by a psychiatrist.

Key words: Clozapine, treatment-resistant schizophrenia, underutilization, outpatients, randomized trial, nurse practitioner. 


\section{Introduction}

Despite evidence for the superiority of clozapine as therapy for treatment-resistant NonAffective Psychotic Disorder (NAPD) (Kane et al. 1988; Siskind et al. 2016; Souza et al. 2013), its prescription rate remains low (Bachmann et al. 2017; Stroup et al. 2014) and clozapine initiation is often delayed (Grover et al. 2015; Howes et al. 2012; Ucok et al. 2015). This delay unnecessarily prolongs patients' suffering and impedes their recovery. Moreover, there is some evidence that a delay may even diminish efficacy of clozapine (Ucok et al. 2015). Important reasons for this delay and under-prescription may be concerns about the safety of clozapine and the need for regular laboratory investigations to prevent potentially dangerous side-effects (Gee et al. 2014; Nielsen et al. 2010; Tungaraza and Farooq 2015). More specifically, the mandatory weekly neutrophil measurements in the first months, to detect agranulocytosis, and the regular monitoring of other sideeffects are time consuming and a burden to both patients and doctors. In a survey, UK professionals considered the deployment of dedicated staff to arrange and monitor this initiation phase as the factor most likely to increase the prescribing of clozapine (Gee et al. 2014). The establishment of specialised teams for the management of patients with treatment-resistant schizophrenia including clozapine treatment in London, increased the number of patients who started to use this drug (Beck et al. 2014). However, the authors acknowledged that there are disadvantages to deploying additional teams: an extra service can cause confusion among clinicians and patients about the clinicians' role and responsibilities, because patients have multiple appointments with different teams of health professionals. With a view to stimulating clozapine use, the aims of this study were to test the following hypotheses: (1) psychiatrists prescribe clozapine more often if they can delegate the monitoring tasks to an advanced nurse practitioner (ANP); (2) monitoring by an ANP is at least as safe as monitoring by a psychiatrist; and (3) delegation of monitoring tasks to an ANP is associated with less frequent premature termination of clozapine in the initial phase (first 18 weeks).

\section{Methods}

\section{Setting / design}

This exploratory study, set up as a cluster-randomized trial (study registration NTR5135), involved Dutch outpatient teams for patients with Non-Affective Psychotic Disorder (NAPD), called Flexible Assertive Community Treatment (FACT) teams. These teams treat patients with severe mental illness and are flexible in that treatment can be intensified in order to prevent the hospitalization of patients during a crisis (van Veldhuizen 2007). FACT teams are responsible for a specific area and their caseload consists of approximately 200-250 outpatients, most of whom have NAPD. In some 
areas, there are also Early Intervention Teams, which treat patients up to 5 years after the first onset of psychosis. These teams differ from FACT teams in that their caseloads are smaller and the patients younger. While teams typically include a psychiatrist, not all teams have an ANP. After at least 2 years of experience in psychiatry, Dutch nurses can follow a 2- or 3-year training programme to become an ANP in mental health care. The profession of ANP in mental health care in the Netherlands resembles that of a mental health ANP in for example the UK, France and Australia and that of a Psychiatric Mental Health Nurse Practitioner in the USA, although in some countries they are authorized to prescribe drugs and in other countries not. In this study, they did not prescribe clozapine. Given the objective of this study, only teams with an ANP were included.

\section{Procedures}

Before randomization, in order to prevent bias, the authors trained the ANPs and psychiatrists of all participating teams for 3 hours about indications for clozapine and monitoring guidelines. Subsequently, the ANP and psychiatrist of each team assessed whether patients had an unmet indication for clozapine, using a standardised procedure (van der Zalm et al. 2018). The decision tree used during this procedure is shown in Appendix 1. The principle investigator (PI) was present at this discussion and available for advice. Thereafter, the ANPs with their corresponding teams were randomized to one of two conditions: A) intervention condition: the ANP performed the somatic screening of patients before clozapine was started, the psycho-education of the patients and their relatives, and the monitoring of laboratory investigations and side-effects; where necessary, they asked supervision from the psychiatrist; or B) treatment as usual: the psychiatrist performed these tasks. In both conditions, the psychiatrist was responsible for the decision to start clozapine and for prescribing it. In order to avoid the assignment of an ANP to both conditions, we decided to randomize the ANPs instead of the teams. Psychiatrists, ANPs, and patients were kept blind to the first hypothesis about the number of patients that would start to use clozapine in each condition. They were only aware of the other two research questions. The randomization was stratified by hospital, geographical area, and FACT vs. Early Intervention Team.

In September 2015, the ANPs randomized to condition A were trained by psychiatrists of the Dutch Clozapine Expert Group and a mental health ANP in two sessions of 3 hours each. The topics covered were: 1) laboratory investigations - their frequency, the interpretation of the results, and the necessary or recommended actions to be taken; 2) dangerous side-effects of clozapine, such as agranulocytosis, myocarditis, and ileus, and how to prevent or detect them; 3) other side-effects such as sedation, orthostasis, constipation, hypersalivation, and metabolic syndrome and how to prevent or treat them; 4) possible interactions between clozapine and other drugs or tobacco use. The participants then had to pass a test of their knowledge. 
All patients who started clozapine between 1 October 2015 and 1 January 2017 were included in this trial. The follow-up of each patient started at the moment of clozapine initiation and lasted 18 weeks, a period in which weekly neutrophil measurements are mandatory in the Netherlands. Patients who started clozapine when in hospital were also included, provided that they were discharged within 18 weeks. We excluded patients who started clozapine during hospital admission and who stayed there during the first 18 weeks. We assumed that for these patients, the decision to start was most often made by the responsible psychiatrist in the hospital. With reference to our second aim, about the safety of the monitoring, inpatient weeks of monitoring were excluded, because the focus of this study was on outpatient clozapine monitoring. The psychiatrist or the ANP informed the PI when clozapine was started. After 18 weeks, the PI visited the ANP or psychiatrist in his or her office. During this visit, the ANP or psychiatrist checked the medical file and provided the following information to the PI: blood assessments (dates and laboratory values) and duration of clozapine use (maximum of 18 weeks). The PI noted this information on structured forms. She asked explicitly for hazardous side-effects and, if clozapine use had been terminated, she documented the reasons for discontinuation. Within this context, it is unlikely that the ANP or psychiatrist invented or concealed outcomes.

\section{Measures}

In order to assess the safety of clozapine monitoring, the PI determined whether the mandatory weekly neutrophil measurements had been performed and registered. If there was an interval of 9 or more days between laboratory investigations, she considered the measurement as missed. We reasoned that the number of missed measurements was an indication of the risk to which the patient was exposed. In addition, we checked the file for reports of dangerous side-effects (e.g., ileus, myocarditis, agranulocytosis, venous thromboembolism) and investigated how soon the ANP or psychiatrist alerted the relevant medical specialist.

\section{Statistical analysis}

Descriptive statistics were used to summarise demographic and clinical characteristics. We used multilevel logistic regression analysis to test for a difference in the proportion of patients who started to use clozapine. As a small number of patients without an NAPD diagnosis also started clozapine, we conducted one analysis with all patients treated by the teams at baseline, regardless of diagnosis, and another analysis restricted to those with an unmet indication for this drug at baseline. In these analyses, patient was the first level and team (the psychiatrist who could prescribe the drug) the second level.

We used a slightly different analysis to test for differences in the number of neutrophil measurements performed. In this analysis, the measurements were the first level, the 
individual patient the second level, and cluster (ANP or psychiatrist) the third level.

The difference in retention on clozapine was analysed using multilevel analysis, with patient as the first level and cluster (ANP or psychiatrist) as the second level. Duration of use was the dependent variable in this analysis. In an additional analysis, we compared the proportion of patients who stopped taking clozapine during the follow-up $\left(\chi^{2}\right.$ test).

All multilevel analyses were random intercept models, adjusted for age, gender, and DSM-IV diagnosis (NAPD vs other diagnoses) as patient-level variables. The second analysis (of neutrophil measurements performed) was a model with random intercept and random slopes on patient level. This model was also adjusted for time (weeks) after clozapine initiation, because neutrophil measurements were more likely to be performed in the first weeks after treatment was started. Descriptive statistics were performed with SPSS, version 22.0. The multilevel analyses were performed with STATA, version 13.0, using procedure GLAMM. A p-value of $<0.05$ was considered statistically significant for all tests.

We calculated the required sample size for a cluster-randomized trial with a power of 0.80 (one-sided testing, $\alpha=0.05$ ). We assumed that there would be at least 15 patients in each cluster with an unmet indication for clozapine (total $n=240$ ), of whom on average $50 \%$ would actually start with this drug $(\mathrm{N}=120)$. We also assumed that in our intervention condition twice as many patients would start with clozapine $(\mathrm{OR}=2)$ and that the coefficient of intracluster correlation was 0.6. The results showed that we needed 8 clusters in each condition (Hayes \& Bennet, 1999).

\section{Results}

\section{Teams and patients}

Four psychiatric institutes in different Dutch regions agreed to participate in this trial. Of the 5 Early Intervention Teams and 29 FACT teams of these institutes, 3 Early Intervention Teams and 20 FACT teams were eligible, see Figure 1. Seventeen ANPs worked for these 23 teams. Some ANPs worked for two teams, but there were no teams with more than one ANP. The ANPs were randomized into one of the two conditions: 9 ANPs, working for 13 teams, were assigned to condition A and 8 ANPs, working for 10 teams, to condition B. At the start of the 15 -month inclusion period, 3839 patients were being treated by these teams. There were no significant differences in mean age or gender between the patients of the two conditions, but there were minor differences in proportions of diagnoses. The baseline characteristics are shown in Table 1. Screening patients for an unmet indication for clozapine at baseline identified 82 patients in 
condition A (3.7\% of all patients) and 91 patients in condition B (5.6\% of all patients), see Figure 1.

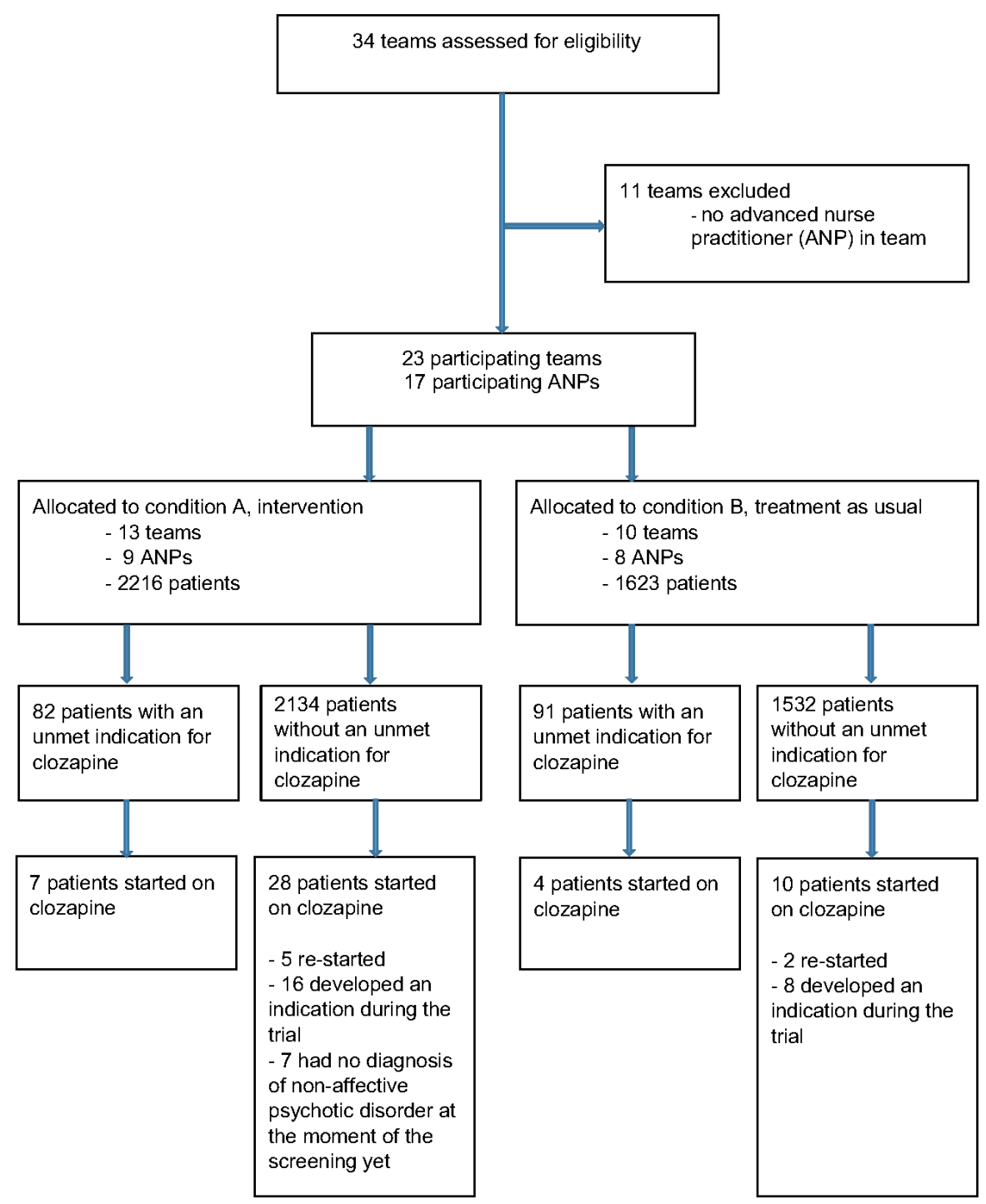

Figure 1. Flow diagram of teams and participants 
Table 1. Baseline characteristics of 3839 patients from 23 teams who participated in a clusterrandomized trial to compare clozapine monitoring by advanced nurse practitioners and psychiatrists.

\begin{tabular}{lrrr}
\hline & $\begin{array}{r}\text { Condition A*, } \\
\text { intervention } \\
\mathbf{N = 2 2 1 6}\end{array}$ & $\begin{array}{r}\text { Condition B**, } \\
\text { treatment as usual } \\
\mathbf{N = 1 6 2 3}\end{array}$ & $\mathbf{P}^{* * *}$ \\
\hline Age, years: mean (s.d.) & $46.6(12.4)$ & $45.9(12.6)$ & .095 \\
Male, n (\%) & $1353(61.2)$ & $1033(63.7)$ & .116 \\
DSM-IV diagnosis, n (\%) & & & .003 \\
Schizophrenia & $885(39.9)$ & $734(45.3)$ & \\
Schizoaffective disorder & $215(9.7)$ & $154(9.5)$ & \\
Schizophreniform disorder & $13(0.6)$ & $17(1.0)$ & \\
Psychotic disorder not otherwise specified & $367(16.6)$ & $258(15.9)$ & \\
Other diagnosis/unknown & $737(33.2)$ & $460(28.3)$ & \\
\hline
\end{tabular}

* Condition A: delegation of clozapine-monitoring tasks to a trained advanced nurse practitioner.

** Condition B: treatment as usual, clozapine monitoring by a psychiatrist.

*** $\chi 2$-test: age, gender, diagnosis (schizophrenia, schizoaffective disorder, schizophreniform disorder, psychotic disorder not otherwise specified, or other diagnosis).

\section{Prescription of clozapine}

Of the 173 patients with an unmet indication for clozapine, only 7 patients in condition A and 4 in condition B were started on clozapine (i.e., $6.4 \%$ of all patients with an unmet indication). The baseline characteristics of these patients are presented in Table 2. The odds ratio for starting clozapine in condition A compared to condition B, adjusted for age and gender was 2.24, CI 0.61-8.21; $\mathrm{p}=.225$.

The reasons for not prescribing clozapine to patients were not systematically studied and this data was not recorded in the files. However, at baseline, psychiatrists and ANPs mentioned reasons for not prescribing clozapine to patients with an indication. A frequently mentioned reason was that they expected the patient not to collaborate with lab exams. Another frequently mentioned reason was non-compliance with oral medication in the past and therefore the need to stay on long-acting injectables. That the patient was doing much better than several years before and starting clozapine was not worth the risk, was also mentioned several times. 
Table 2. Sociodemographic and clinical characteristics of patients who started clozapine in a cluster-randomized trial comparing clozapine monitoring by advanced nurse practitioners and psychiatrists.

\begin{tabular}{|c|c|c|c|c|}
\hline \multirow[t]{2}{*}{ Characteristic } & \multicolumn{2}{|c|}{$\begin{array}{c}\text { Starters with indication } \\
\text { at baseline } \\
\mathrm{N}=11\end{array}$} & \multicolumn{2}{|c|}{$\begin{array}{c}\text { Starters with indication at } \\
\text { baseline or thereafter } \\
\mathrm{N}=49\end{array}$} \\
\hline & $\begin{array}{r}\text { Condition } \\
\mathbf{A}^{*}, \\
\text { intervention } \\
(\mathbf{n}=7)\end{array}$ & 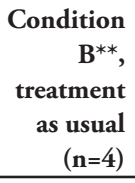 & $\begin{array}{r}\text { Condition } \\
\mathbf{A}^{*}, \\
\text { intervention } \\
(\mathbf{n}=35)\end{array}$ & 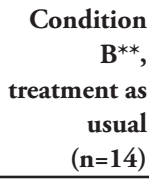 \\
\hline Age, years: mean (s.d.) & $48.1(3.1)$ & $55.5(6.4)$ & $45.7(12.3)$ & $45.6(14.0)$ \\
\hline Male, n (\%) & $4(57.1)$ & $2(50.0)$ & $22(62.9)$ & $11(78.6)$ \\
\hline \multicolumn{5}{|l|}{ DSM-IV diagnosis, n (\%) } \\
\hline Schizophrenia & $4(57.1)$ & $1(25.0)$ & $20(57.1)$ & $9(64.3)$ \\
\hline Schizoaffective disorder & $2(28.6)$ & $2(50.0)$ & $3(8.6)$ & $2(14.3)$ \\
\hline Schizophreniform disorder & & & - & \\
\hline Psychotic disorder not otherwise specified & $1(14.3)$ & $1(25.0)$ & $7(20.0)$ & $3(21.4)$ \\
\hline Other diagnosis & & & $5(14.3)$ & \\
\hline
\end{tabular}

* Condition A: delegation of clozapine-monitoring tasks to a trained advanced nurse practitioner.

** Condition B: treatment as usual, clozapine monitoring by a psychiatrist.

*** $\chi 2$-test: age, gender, diagnosis (schizophrenia, schizoaffective disorder, schizophreniform disorder, psychotic disorder not otherwise specified, or other diagnosis).

\section{Additional analysis on prescription of clozapine}

Apart from the patients with an unmet indication for clozapine at baseline, there were other patients in the teams who started with clozapine. Those patients either re-started the drug, developed an indication during the trial (due to an increase of positive symptoms or to a lack of effect of other antipsychotics), or did not have an NAPD diagnosis at baseline (see Figure 1). In total, 49 started on clozapine during the study period: 35 in condition A and 14 in condition B. Table 2 shows the characteristics of these patients. Taking all 3,839 patients into account, the odds ratio for starting clozapine in condition A compared to condition B, adjusted for age, gender and NAPD-diagnosis (yes/no) was 1.90 (95\% CI: 0.93-3.87; $\mathrm{p}=.078)$.

There were large differences between the teams in prescribing clozapine, see supplementary Table S1. Psychiatrists who had spoken negatively about clozapine hardly prescribed it, regardless of the condition they were in. Conversely, psychiatrists with a strong positive attitude toward clozapine regularly prescribed it, also regardless of the condition they were in. On the other hand, some psychiatrists in condition A collaborating with three ANPs informed us that they were very glad that they were allocated to the intervention condition, because now they could start with ambulatory clozapine initiation. They stated that they had not prescribed clozapine if they had been allocated to condition 
B. Supplementary table S1 shows the differences per team in patients on clozapine, with an indication for clozapine and who started with this drug. This table also shows that there were more patients newly admitted to the ambulatory team during the study period who started to use clozapine in condition $A(N=13)$ compared to condition $B$ $(\mathrm{N}=4)$. There was an in- and out-flow of patients during the inclusion period and it was not possible to keep track of all these changes. Nonetheless, differences between the conditions were in line with the first hypothesis.

\section{Safety of clozapine monitoring}

For our second question on safety of clozapine monitoring, we included all patients who started clozapine in the participating teams $(n=49)$. In condition $A, 8$ patients started clozapine as an inpatient and another patient was admitted twice during the first 18 weeks. The mean duration of admission, for these 9 patients, was 6.9 weeks (SD 3.8). In condition $B, 7$ patients started clozapine as inpatients. Their mean duration of admission was 8.7 weeks (SD 4.9). After the exclusion of the weeks of inpatient treatment (mean 1.8 weeks in condition A and 4.4 weeks in condition B) and the time between premature stopping of clozapine and the end of follow-up, neutrophil measurement for the 49 included patients was mandatory for 682 weeks (517 in Condition A and 165 in condition B). Overall, 368 neutrophil measurements in condition A were performed as required (71.2\%) and 111 in condition $\mathrm{B}(67.3 \%)$ (OR, adjusted for age, gender, and weeks after start $0.98 ; 95 \% \mathrm{CI}, 0.16-6.04 ; \mathrm{p}=.982)$. The proportion of neutrophil measurements carried out by one ANP or psychiatrist varied considerably. In condition A, this proportion ranged from 30.6 to $87.2 \%$ and in condition B from 0 to $97 \%$. Supplementary Table S2 shows these proportions per cluster. No dangerous side-effects occurred in either condition. The reasons for missed neutrophil counts varied. In most cases the patients received a laboratory form, but did not go to the laboratory. In one particular area neutrophil measurements were missed because of a failing laboratory. For example, the wrong tests were performed or the laboratory assistant went to the wrong address. Holidays of patients were another reason for missed lab exams. A psychiatrist failed to notice that one patient missed all laboratory tests. Missing laboratory exams was only in one patient the reason to stop clozapine. This psychiatrist made the decision when the patient had a fever and persisted in refusing neutrophil measurements.

\section{Duration of clozapine use}

For the analysis on duration of use, we also included all patients who started clozapine in the participating teams $(n=49)$. There were no significant differences in the retention on clozapine - the mean duration of use (including inpatient weeks of use)was 16.53 (SD 4.5) weeks in condition A and 15.96 (SD 3.4) weeks in condition $B(b=0.31 ; 95 \%$ CI: -2.26-2.88; $\mathrm{p}=.815)$. In condition A, $11.4 \%$ of the clozapine starters stopped taking the drug prematurely $\left(<18\right.$ weeks) compared to $28.6 \%$ in condition $B\left(\chi^{2}=2.15 ; \mathrm{df}=1\right.$; 
$\mathrm{p}=.142$ ). One patient in condition B stopped to use clozapine after 7 weeks because it was not effective and one patient in condition A had to stop clozapine because of a fever in combination with the refusal to go to the laboratory. In all other patients $(n=6)$, the reason for discontinuation were the side-effects of clozapine. This was a shared decision for all patients except one.

\section{Discussion}

\section{Main findings}

We tested the hypotheses that psychiatrists would prescribe clozapine more often if they could delegate the monitoring tasks to an ANP, that monitoring by an ANP is at least as safe as monitoring by a psychiatrist, and that delegation of monitoring tasks to an ANP is associated with a longer retention on clozapine. Our findings were consistent with the first hypothesis, but failed to reach the conventional level of statistical significance, most likely due to a lack of statistical power. The OR was close to the OR assumed in our power calculation, but the number of patients with an unfulfilled indication for clozapine was smaller than we expected. In addition, the number of patients who started with this drug in either condition were much smaller than expected. We conclude that even when an ANP is present for support, Dutch psychiatrists still fail to start clozapine for the vast majority of patients identified as having potential benefit from clozapine. We can only speculate about the causes of this hesitation. Possible reasons are the sideeffects of clozapine, some of which are dangerous and require a prompt and adequate reaction, or an absence of trust in the potential benefits from this drug.

Clozapine monitoring by an ANP seems as safe, in terms of performed and recorded neutrophil measurements, as that done by a psychiatrist. Patients monitored by an ANP tended to stay on treatment for longer than patients monitored by a psychiatrist, but the difference was small and statistically not significant.

\section{Comparison with other studies}

This study was the first randomized controlled trial to examine the effect of an intervention to stimulate the use of clozapine. The findings of our study are in line with those of the study of Goren et al. (2016). In their study, Goren et al interviewed psychiatrists over the phone to identify facilitators of and barriers to clozapine use. They concluded that the involvement of ANPs and clinical pharmacists in clozapine teams was associated with high clozapine prescription rates. This multidisciplinary approach is comparable to the ANP condition in our study, where all ANPs collaborated with a psychiatrist. As for the mandatory weekly neutrophil measurements, it is difficult to compare the results between different settings. Of note, in the Netherlands, there is no manufacturer- 
organised mandatory service or database for haematological monitoring. It is the responsibility of the physician to organise these weekly laboratory investigations. To our knowledge, only one other study reported the frequency of neutrophil measurements after the initiation of clozapine, with measurements being performed during the first 18 weeks at a mean interval of 25 days (Ingimarsson et al. 2016). This is less often than in our study.

In order to compare sole nurse-led clozapine services to physician-led teams, Gage et al. (2015) interviewed patients and concluded that clinics run by a nurse could effectively provide clozapine-monitoring services. However, the lack of direct access to a physician led to an increased use of community psychiatric services and to more hospital psychiatrist appointments. This argues for a multidisciplinary approach within one team, as occurred in the ANP condition in our study.

\section{Strengths and limitations}

A strength of this study is that all patients, psychiatrists, and ANPs were (and remained) blind to the first hypothesis. Another strength is that this real-world study involved patients and healthcare professionals from a non-academic setting, which is representative for many European services. Additionally, multidisciplinary outpatient teams like the FACT-teams in the Netherlands are comparable to services in other European countries (Rosenheck et al. 2016; Valdes-Stauber et al. 2014). However, some limitations need to be addressed. First, since the proportion of patients starting clozapine was smaller than expected, especially among those with an indication at baseline, the power of the trial to address the research questions was insufficient. Although we did not approach our second question as a non-inferiority analysis, the results do not indicate that the monitoring in our intervention condition was less safe. Second, the appraisal of the safety of the monitoring was limited to the number of neutrophil measurements performed and to the reporting of dangerous side-effects in the patient files. Information on whether the results of the laboratory investigations were checked in time is usually not recorded. It was not possible to investigate whether other aspects, such as constipation and blood pressure, were monitored as required by guidelines. Third, the data-collection was not performed by blinded research assistants. We believe that asking permission for an independent researcher to check the file, would have lowered the number of participants, because many patients are hesitant to start on clozapine and some of them are paranoid. In order to prevent bias, the PI was present at the moment the ANP or the psychiatrist checked the files for the data-collection. Fourth, in condition A, there was a collaboration between ANP and psychiatrists, which may have been an advantage. However, since ANPs cannot be responsible for the total of clozapine care, a small involvement of a psychiatrist, as in our condition A, corresponds to reality. Fifth, the training of psychiatrists and ANPs preceding the trial and the assessment of patients 
for an unmet indication for clozapine, could have increased the number of patients to start with clozapine. However, the number of patients that started was much lower than expected and this effect should be the same for both conditions. In addition, the knowledge of being in a study on safety of clozapine monitoring may have increased the number of lab exams. Since both psychiatrists and ANPs indicated that they did not want to be inferior to those in the other condition, we expected this effect to be similar in the conditions. Sixth, we were unable to adjust for the availability of a point-of-care (POC) device to test neutrophils, because only one team was in possession of such a device at the start of the follow-up (a team in condition B). Bogers et al. (2015) found that patients preferred POC testing and that this method moderately influenced their motivation for clozapine therapy. The availability of POC testing could, therefore, have led to more patients starting with clozapine and to a longer retention. Finally, the results of this study are only generalizable to countries where prescribers are responsible for clozapine monitoring, so without an independent clozapine monitoring agency.

\section{Implications}

The results of this trial show that identifying patients with an indication for clozapine does not automatically lead to improved prescription rates. The results also suggest that some prescribers do not prescribe clozapine, irrespective of the condition they were in. In future research on interventions to stimulate use of clozapine, the attitude of the prescriber may be a better target for interventions. However, given the odds ratio and the p-value found in this small sample, we are confident that the use of clozapine can be stimulated by delegating the labour-intensive monitoring tasks to an ANP without compromising safety. This strategy can lead to earlier recovery from chronic psychosis and better patient outcome.

\section{Acknowledgements}

The authors would like to thank all participating psychiatrists and advanced nurse practitioners for helping to provide the data necessary for this study.

\section{Group information:}

The CLOZ-NP Study Group members include Bert Luteijn, Marion Hageman and Roelie Molenaar, Rivierduinen Institute for Mental Health, Gouda, Leidschendam and Leiden, The Netherlands; Hetty Pronk-Verweij and Tine Hendriks, Institute for Mental Health Care Eindhoven (GGzE), Eindhoven, The Netherlands; Harm Gijsman and Raymond Brandt, Pro Persona Mental Healthcare, Nijmegen, The Netherlands; Ruud Feijen, Arkin Mental Health Care, Amsterdam, The Netherlands. 


\section{Compliance with ethical standards}

This research was conducted in accordance with the 1964 Helsinki Declaration and its later amendments. The study was reviewed by the Medical Ethics Committee of Leeuwarden, the Netherlands. This Committee decided that the Medical Research Involving Human Subjects Act did not apply to our study and that an official approval of this study by the committee was not required. Informed consent for clozapine initiation was not asked, because treatment with clozapine is according to Dutch guidelines and because both psychiatrists and ANPs are authorised to perform the monitoring. The authors declare that they have no conflict of interest and that this research did not receive any specific grant from funding agencies in the public, commercial or not-forprofit sectors. 


\section{References}

Bachmann, C. J., Aagaard, L., Bernardo, M., Brandt, L., Cartabia, M., Clavenna, A., et al. (2017). International trends in clozapine use: a study in 17 countries. Acta Psychiatrica Scandinavica, 136(1), 37-51. doi:10.1111/acps.12742

Beck, K., McCutcheon, R., Bloomfield, M. A., Gaughran, F., Reis Marques, T., MacCabe, J., et al. (2014). The practical management of refractory schizophrenia--the Maudsley Treatment REview and Assessment Team service approach. Acta Psychiatrica Scandinavica, 130(6), 427-438. doi:10.1111/ acps. 12327

Bogers, J. P., Bui, H., Herruer, M., \& Cohen, D. (2015). Capillary compared to venous blood sampling in clozapine treatment: patients' and healthcare practitioners' experiences with a point-of-care device. European Neuropsychopharmacology, 25(3), 319-324.

Gage, H., Family, H., Murphy, F., Williams, P., Sutton, J., \& Taylor, D. (2015). Comparison of sole nurse and team-delivered community clozapine services for people with treatment-resistant schizophrenia. Journal of Advanced Nursing, 71(3), 547-558. doi:10.1111/jan.12527

Gee, S., Vergunst, F., Howes, O., \& Taylor, D. (2014). Practitioner attitudes to clozapine initiation. Acta Psychiatrica Scandinavica, 130(1), 16-24. doi:10.1111/acps.12193

Goren, J. L., Rose, A. J., Engle, R. L., Smith, E. G., Christopher, M. L., Rickles, N. M., et al. (2016). Organizational Characteristics of Veterans Affairs Clinics With High and Low Utilization of Clozapine. Psychiatric Services,, 67(11), 1189-1196. doi:10.1176/appi.ps.201500506

Grover, S., Hazari, N., Chakrabarti, S., \& Avasthi, A. (2015). Delay in initiation of clozapine: a retrospective study from a tertiary care hospital in North India. Psychiatry Research, 226(1), 181185. doi:10.1016/j.psychres.2014.12.046

Hayes, R. J., \& Bennett, S. (1999). Simple sample size calculation for cluster-randomized trials. International journal of epidemiology, 28(2), 319-326.

Howes, O. D., Vergunst, F., Gee, S., McGuire, P., Kapur, S., \& Taylor, D. (2012). Adherence to treatment guidelines in clinical practice: study of antipsychotic treatment prior to clozapine initiation. The British Journal of Psychiatry, 201(6), 481-485. doi:10.1192/bjp.bp.111.105833

Ingimarsson, O., MacCabe, J. H., Haraldsson, M., Jonsdottir, H., \& Sigurdsson, E. (2016). Neutropenia and agranulocytosis during treatment of schizophrenia with clozapine versus other antipsychotics: an observational study in Iceland. BMC Psychiatry, 16(1), 441. doi:10.1186/s12888-016-1167-0

Kane, J., Honigfeld, G., Singer, J., \& Meltzer, H. (1988). Clozapine for the treatment-resistant schizophrenic. A double-blind comparison with chlorpromazine. Archives of General Psychiatry, 45(9), 789-796.

Nielsen, J., Dahm, M., Lublin, H., \& Taylor, D. (2010). Psychiatrists' attitude towards and knowledge of clozapine treatment. Journal of Psychopharmacology, 24(7), 965-971. doi:10.1177/0269881108100320

Rosenheck, R., Leslie, D., Sint, K., Lin, H., Robinson, D. G., Schooler, N. R., et al. (2016). CostEffectiveness of Comprehensive, Integrated Care for First Episode Psychosis in the NIMH RAISE Early Treatment Program. Schizophrenia Bulletin, 42(4), 896-906. doi:10.1093/schbul/sbv224

Siskind, D., McCartney, L., Goldschlager, R., \& Kisely, S. (2016). Clozapine v. first- and second-generation 
antipsychotics in treatment-refractory schizophrenia: systematic review and meta-analysis. The British Journal of Psychiatry, 209(5), 385-392. doi:10.1192/bjp.bp.115.177261

Souza, J. S., Kayo, M., Tassell, I., Martins, C. B., \& Elkis, H. (2013). Efficacy of olanzapine in comparison with clozapine for treatment-resistant schizophrenia: evidence from a systematic review and metaanalyses. CNS Spectrums, 18(2), 82-89. doi:10.1017/s1092852912000806

Stroup, T. S., Gerhard, T., Crystal, S., Huang, C., \& Olfson, M. (2014). Geographic and clinical variation in clozapine use in the United States. Psychiatric Services, 65(2), 186-192. doi:10.1176/appi. ps. 201300180

Tungaraza, T. E., \& Farooq, S. (2015). Clozapine prescribing in the UK: views and experience of consultant psychiatrists. Therapeutic Advances in Psychopharmacology, 5(2), 88-96. doi:10.1177/2045125314566808

Ucok, A., Cikrikcili, U., Karabulut, S., Salaj, A., Ozturk, M., Tabak, O., et al. (2015). Delayed initiation of clozapine may be related to poor response in treatment-resistant schizophrenia. International Clinical Psychopharmacology, 30(5), 290-295. doi:10.1097/yic.0000000000000086

Valdes-Stauber, J., Putzhammer, A., \& Kilian, R. (2014). [Decentralized outpatient teams in communitybased psychiatric care: comparison of two Bavarian rural catchment areas]. Der Nervenarzt, 85(5), 596-605. doi:10.1007/s00115-013-3836-2

van der Zalm, Y. C., Termorshuizen, F., Schulte, P. F., Bogers, J. P., Marcelis, M., Sommer, I. E., et al. (2018). Prescription and Underprescription of Clozapine in Dutch Ambulatory Care. Frontiers in Psychiatry, 9, 231. doi:10.3389/fpsyt.2018.00231

van Veldhuizen, J. R. (2007). FACT: a Dutch version of ACT. Community Mental Health Journal, 43(4), 421-433. doi:10.1007/s10597-007-9089-4 


\section{Supplementary material}

Supplementary Table S1. The numbers of patients with an indication for clozapine and of those who started with this drug.

\begin{tabular}{|c|c|c|c|c|c|c|c|}
\hline $\begin{array}{l}\text { Condition } \mathrm{A}^{*} \text {, } \\
\text { intervention }\end{array}$ & $\begin{array}{l}\text { Patients, } \\
\text { any } \\
\text { diagnosis } \\
\mathrm{N}=2216\end{array}$ & $\begin{array}{l}\text { Patients } \\
\text { with } \\
\text { NAPD** } \\
\text { N=1451 }\end{array}$ & $\begin{array}{l}\begin{array}{l}\text { On } \\
\text { clozapine }\end{array} \\
\mathrm{N}=319(\%)\end{array}$ & $\begin{array}{l}\text { With } \\
\text { indication } \\
\text { for this } \\
\text { drug } \\
\mathrm{N}=82(\%)\end{array}$ & 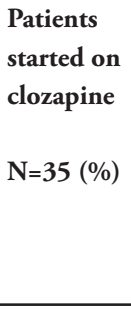 & $\begin{array}{l}\text { Started with } \\
\text { indication } \\
\text { at baseline. } \\
\mathrm{N}=7\end{array}$ & $\begin{array}{l}\text { Newly } \\
\text { admitted } \\
\text { patients } \\
\text { who } \\
\text { started (not } \\
\text { included in } \\
\text { study) } \\
\mathrm{N}=13\end{array}$ \\
\hline ANP 1 & 174 & 122 & $26(21)$ & $9(7)$ & $5(4)$ & 1 & - \\
\hline ANP 2 & 191 & 117 & $24(21)$ & $2(2)$ & $1(1)$ & - & - \\
\hline ANP 3 & 334 & 194 & $63(32)$ & $4(2)$ & $2(1)$ & - & 1 \\
\hline ANP 4 & 391 & 211 & $32(15)$ & $15(7)$ & $11(5)$ & 1 & 3 \\
\hline ANP 5 & 215 & 125 & $22(18)$ & $4(3)$ & $4(3)$ & - & 1 \\
\hline ANP 6 & 332 & 281 & $74(26)$ & $16(6)$ & $9(3)$ & 3 & 1 \\
\hline ANP 7 & 346 & 267 & $63(24)$ & $24(9)$ & $3(1)$ & 2 & 6 \\
\hline ANP 8 & 145 & 91 & $10(11)$ & $7(8)$ & - & - & 1 \\
\hline ANP $9^{* * *}$ & 88 & 71 & $6(8)$ & $1(1)$ & - & - & - \\
\hline $\begin{array}{l}\text { Condition } \mathrm{B}^{* *} \text {, } \\
\text { treatment as usual }\end{array}$ & $\mathrm{N}=1623$ & $\mathrm{~N}=1163$ & $\mathrm{~N}=243(\%)$ & $\mathrm{N}=91(\%)$ & $\mathrm{N}=14(\%)$ & $\mathrm{N}=4$ & $\mathrm{~N}=4$ \\
\hline Psychiatrist 1 & 183 & 134 & $37(28)$ & $16(12)$ & $4(3)$ & 2 & - \\
\hline Psychiatrist 2 & 216 & 129 & $24(19)$ & $20(16)$ & $2(2)$ & 1 & 1 \\
\hline Psychiatrist 3 & 170 & 91 & $13(14)$ & $6(7)$ & $1(1)$ & 1 & - \\
\hline Psychiatrist 4 *** & 102 & 92 & $13(14)$ & - & $2(2)$ & - & 1 \\
\hline Psychiatrist 5 & 488 & 375 & $95(25)$ & $21(6)$ & $1(0.2)$ & - & - \\
\hline Psychiatrist $6^{* * *}$ & 167 & 140 & $15(11)$ & $14(10)$ & $2(1)$ & - & - \\
\hline Psychiatrist 7 & 146 & 98 & $18(18)$ & $10(10)$ & $2(2)$ & - & 1 \\
\hline Psychiatrist 8 & 151 & 104 & $28(27)$ & $4(4)$ & - & - & 1 \\
\hline
\end{tabular}

* Condition A: delegation of clozapine-monitoring tasks to a trained advanced nurse practitioner Condition B: treatment as usual, clozapine monitoring by a psychiatrist

** Non Affective Psychotic Disorder

*** Early intervention team 
Supplementary Table S2. The numbers of patients who started on clozapine and the numbers of (mandatory and performed) neutrophil measurements, per condition and by each advanced nurse practitioner (Condition A) and psychiatrist (Condition B).

\begin{tabular}{llll}
\hline Condition $\mathbf{A}^{*}$, intervention & $\begin{array}{l}\text { Patients started on } \\
\text { clozapine }\end{array}$ & $\begin{array}{l}\text { Mandatory neutrophil } \\
\text { measurements } \\
\mathbf{N = 5 1 7}\end{array}$ & $\begin{array}{l}\text { Neutrophil } \\
\text { measurements, } \\
\text { performed on time (\%) } \\
\mathbf{N}=\mathbf{3 6 8}(\mathbf{7 1 . 2})\end{array}$ \\
\hline ANP 1 & 5 & 77 & $66(85.7)$ \\
ANP 2 & 1 & 14 & $7(50)$ \\
ANP 3 & 2 & 36 & $11(30.6)$ \\
ANP 4 & 11 & 157 & $114(72.6)$ \\
ANP 5 & 4 & 42 & $15(35.7)$ \\
ANP 6 & 9 & 144 & $114(79.2)$ \\
ANP 7 & 3 & 47 & $41(87.2)$ \\
ANP 8 & - & - & - \\
ANP 9 & - & - & - \\
\hline
\end{tabular}

\begin{tabular}{llll}
\hline $\begin{array}{l}\text { Condition } \mathbf{B}^{* *} \text {. } \\
\text { treatment as usual }\end{array}$ & $\begin{array}{l}\text { Patients started on } \\
\text { clozapine }\end{array}$ & $\begin{array}{l}\text { Mandatory neutrophil } \\
\text { measurements } \\
\mathbf{N = 1 6 5}\end{array}$ & $\begin{array}{l}\text { Neutrophil } \\
\text { measurements, } \\
\text { performed on time (\%) } \\
\mathbf{N = 1 1 1}(\mathbf{6 7 . 3})\end{array}$ \\
\hline Psychiatrist 1 & 4 & 38 & $26(68.4)$ \\
Psychiatrist 2 & 2 & 38 & $34(89.5)$ \\
Psychiatrist 3 & 1 & 9 & $1(11.1)$ \\
Psychiatrist 4 & 2 & 27 & $20(74.1)$ \\
Psychiatrist 5 & 1 & 5 & $0 * * *(0)$ \\
Psychiatrist 6 & 2 & 19 & $2(10.5)$ \\
Psychiatrist 7 & 2 & 29 & $28(96.6)$ \\
Psychiatrist 8 & - & - & - \\
\hline
\end{tabular}

* Condition A: delegation of clozapine-monitoring tasks to a trained advanced nurse practitioner.

** Condition B: treatment as usual, clozapine monitoring by a psychiatrist.

*** The psychiatrist instructed the patient to visit a laboratory, for blood tests, but he failed to notice that the patient did not follow this instruction. 


\section{Appendix}

\section{Adequate dosages, description of the scores on the Clinical Global Impression-Schizophrenia Scale (CGI-SCH), and the decision tree.}

Adequate dosage, oral medication

\begin{tabular}{lr}
\hline Drug & Adequate dosage \\
\hline Aripiprazole & $15 \mathrm{mg} / \mathrm{d}(2)$ \\
Bromperidol & $4 \mathrm{mg} / \mathrm{d}$ \\
Flupentixol & $4 \mathrm{mg} / \mathrm{d}$ \\
Haloperidol & $4 \mathrm{mg} / \mathrm{d}(3)$ \\
Lurasidone & $40 \mathrm{mg} / \mathrm{d}(7)$ \\
Olanzapine & $15 \mathrm{mg} / \mathrm{d}(2)$ \\
Paliperidone & $6 \mathrm{mg} / \mathrm{d}(5)$ \\
Penfluridol & $40 \mathrm{mg} / \mathrm{wk}(5)$ \\
Perphenazine & $16 \mathrm{mg} / \mathrm{d}(2)$ \\
Pimozide & $4 \mathrm{mg} / \mathrm{d}(5)$ \\
Quetiapine & $400 \mathrm{mg} / \mathrm{d}(2)$ \\
Risperidone & $3 \mathrm{mg} / \mathrm{d}(2)$ \\
Sertindole & $12-20 \mathrm{md} / \mathrm{d}(6)$ \\
Sulpiride & $800 \mathrm{mg} / \mathrm{d}(5)$ \\
Zuclopenthixol & $16 \mathrm{mg} / \mathrm{d}(4)$ \\
\hline
\end{tabular}

Adequate dosage, long lasting injectables

\begin{tabular}{|c|c|}
\hline Drug & Adequate dosage \\
\hline Aripiprazole & $400 \mathrm{mg} / 4 \mathrm{wk}(6)$ \\
\hline Bromperidol & $100 \mathrm{mg} / 4 \mathrm{wk}$ \\
\hline Fluphenazine & $50 \mathrm{mg} / 4 \mathrm{wk}(1)$ \\
\hline Flupentixol & $40 \mathrm{mg} / 2 \mathrm{wk}(1)$ \\
\hline Fluspirilene & $4 \mathrm{mg} / \mathrm{wk}(1)$ \\
\hline Haloperidol & $100 \mathrm{mg} / 4 \mathrm{wk}(1)$ \\
\hline Olanzapine & $210 \mathrm{mg} / 2 \mathrm{wk}(6)$ \\
\hline Paliperidone & $75 \mathrm{mg} / 4 \mathrm{wk}(6)$ \\
\hline Risperidone & $37,5 \mathrm{mg} / 2 \mathrm{wk}(1)$ \\
\hline Zuclopenthixol & $225 \mathrm{mg} / 3 \mathrm{wk}$ (1) \\
\hline \multicolumn{2}{|c|}{ (1) Moleman P, Birkenhäger T. Praktische Psychofarmacologie 2009. Houten: Bohn Stafleu Van Loghum. } \\
\hline \multicolumn{2}{|c|}{ (2) Lieberman J et al. New England Journal of Medicine 2005;353(12):1209-1223. } \\
\hline \multicolumn{2}{|c|}{ (3) Andreasen $\mathrm{N}$ et al. Biological psychiatry 2010;67(3):255-262. } \\
\hline \multicolumn{2}{|c|}{ (4) Van Alphen C et al. Multidisciplinaire richtlijn schizofrenie 2012. Utrecht: De Tijdstroom. } \\
\hline \multicolumn{2}{|c|}{ (5) http://www.whocc.no/atc_ddd_index/?code=N05A } \\
\hline \multicolumn{2}{|c|}{ (6) Van Loenen A. Farmacotherapeutisch Kompas 2003. Amstelveen. } \\
\hline \multicolumn{2}{|c|}{ (7) Loebel A et al. European Psychiatry 2015;30(1):26-31. } \\
\hline
\end{tabular}

N.B. If the dosage was lower or the period shorter, due to untreatable EPS, this counts as adequate treatment. 


\section{Description of the scores on the Clinical Global Impression-Schizophrenia Scale (CGI-SCH).}

$1=$ Normal-not at all ill, symptoms of disorder not present past seven days.

$2=$ Borderline mentally ill-subtle or suspected pathology.

3=Mildly ill-clearly established symptoms with minimal, if any, distress or difficulty in social and occupational function.

4=Moderately ill-overt symptoms causing noticeable, but modest, functional impairment or distress, symptom level may warrant medication.

5=Markedly ill-intrusive symptoms that distinctly impair social/occupational function or cause intrusive levels of distress.

$6=$ Severely ill-disruptive pathology, behavior and function are frequently influenced by symptoms, may require assistance from others.

7=Among the most extremely ill patients-pathology drastically interferes in many life functions; may be hospitalized. 


\section{Decision tree}

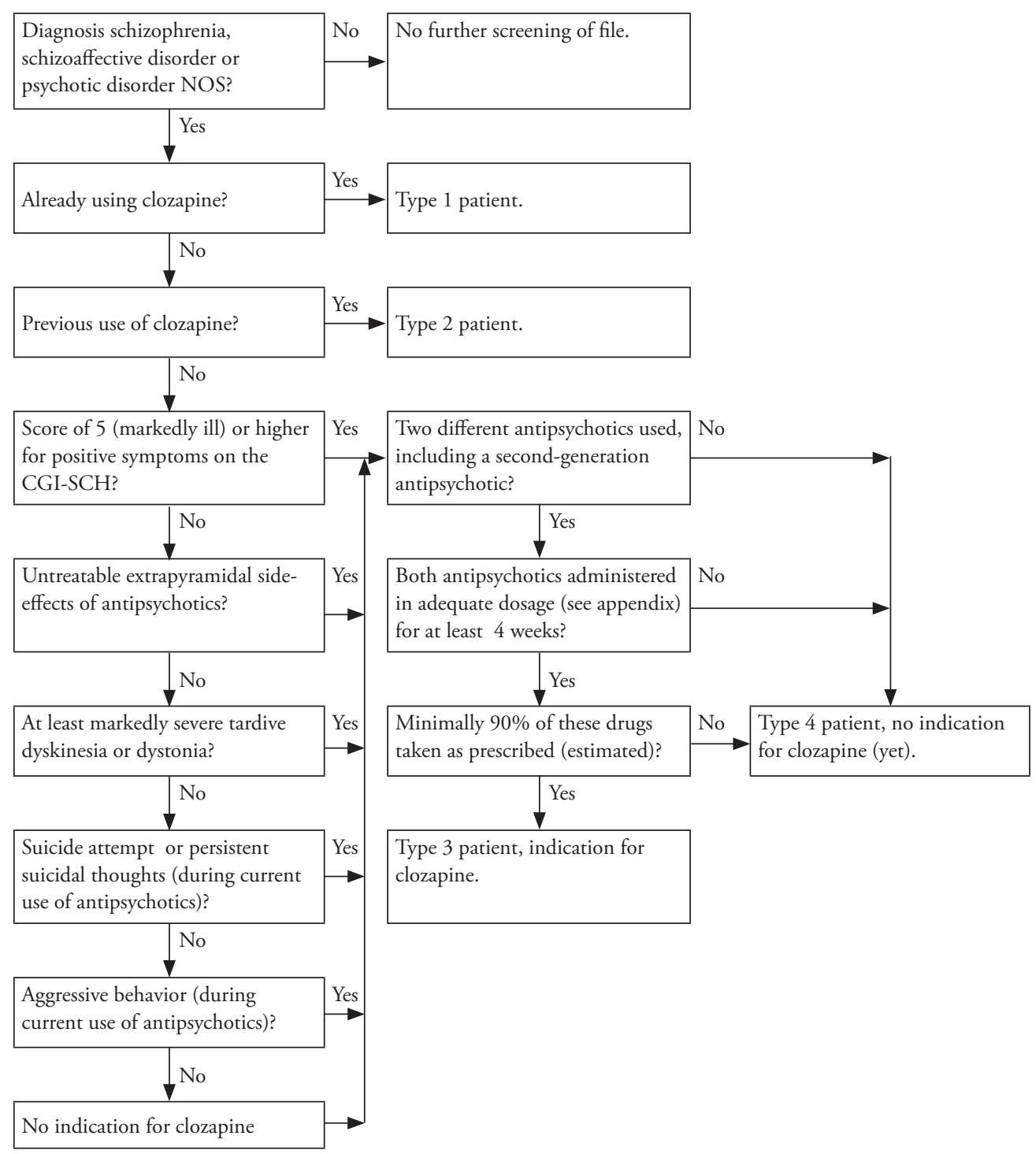




\section{PART II}

Clozapine and mortality 

Chapter 4 
In view of the high all-cause mortality among patients with schizophrenia and the wide range of positive and negative effects of clozapine, it is important to know whether the drug is associated with a differential mortality pattern in comparison to other antipsychotic drugs. Tiihonen et al. (2009) reported a significantly lowered all-cause mortality and a lowered mortality from suicide in users of clozapine. In this journal, however, de Hert et al. (2010) pointed out that several types of bias may have influenced the results. For example, the exclusion of deaths after a hospitalization of more than 2 days, so that two-thirds of deaths were not considered. Another example is survivorship bias in the analysis of death by suicide. Suicide is more common in the first years after onset of psychosis, whereas clozapine is often first prescribed years later.

The purpose of this letter is to discuss biases and methodological errors in other studies that reported a significantly decreased mortality associated with clozapine. The need to do so arose with the recent publication of a meta-analysis (Vermeulen et al., 2018), which concluded that long-term all-cause mortality was substantially lower with continuous clozapine treatment than with treatment with other antipsychotics (mortality rate ratio $=0.56 ; 95 \%$ CI $0.36-0.85$ ). The authors used crude, unadjusted mortality rates in the meta-analysis and ignored the fact that in many of the reviewed studies there is a considerable age difference between clozapine-users and non-users (see supplementary Table 1). The comparison of a relatively young group of clozapine-users to an older group of non-users of this drug, without adjusting for age, resulted in an overly optimistic evaluation of clozapine and an unjustified conclusion. For illustration, supplementary Table 2 shows the large differences between the crude and the adjusted mortality rate ratios of the included studies.

Besides the study of Tiihonen et al. (2009), two other studies reported a significantly lower mortality rate with clozapine than with other antipsychotics. Hayes et al. (2015) reported a strong association between being prescribed clozapine and a lower mortality (adjusted hazard ratio $=0.4 ; 95 \%$ CI $0.2-0.7$ ). However, the authors failed to apply a left truncation of the time between the start of follow-up (the time of diagnosis) and when clozapine was started. Therefore, the patient was not observable for risk of death in this period implying long periods necessarily without any observed death event ('immortal time bias').

Perhaps the best study in this area was conducted by Walker et al. (1997). They compared episodes of clozapine use to episodes after discontinuation of this drug and divided the time period after discontinuation into episodes of "recent use" (up to 3 months after discontinuation) and episodes of "past use" (more than three months later). All-cause mortality was significantly lower during episodes of use than during episodes of past use. It was highest during episodes of recent use, probably because moribund patients 
were taken off the drug. An impressive finding was the lower risk of suicide during current use than during past use (standardized mortality ratio $=0.17$; $95 \%$ CI 0.10 0.30). Nevertheless, the study was limited by its design (one would have preferred a comparison of clozapine users to treatment-resistant patients who do not receive this drug) and the lack of information on the reasons why clozapine was discontinued and the pharmacotherapy after this discontinuation.

New large-scale and long-term studies are needed that take important forms of bias into account. In order to avoid the risk of survivorship bias, we recommend the use of incidence cohorts, especially if death by suicide is to be investigated. As to confounding by indication, new studies should take into account that clozapine might be prescribed more often to relatively healthy patients, and that the intensive monitoring of patients may lead to better somatic treatment. It is also worthwhile to note that the inclusion of individuals who do not use any antipsychotic affects the results. Wimberley et al. (2017) found a significantly higher adjusted hazard ratio for all-cause mortality among clozapine non-users ( $\mathrm{HR}=1.84$; 95\% CI 1.13-3.01). However, after distinguishing between users and non-users of antipsychotics, the hazard ratio for all-cause mortality was no longer significantly higher among users of other antipsychotics (HR=1.41;95\% CI $0.83-2.40)$ whereas it was significantly higher among non-users of antipsychotics (HR=2.46; 95\% CI 1.46-4.14).

The good news, despite all these caveats and criticisms, is that to the best of our knowledge no study has reported a significantly increased mortality among clozapine users. Thus, it is possible that the marked therapeutic effect of clozapine outweighs the negative effects of metabolic and other side effects. Indeed many patients report that they have finally found peace after years of agony and it is conceivable that this decrease in stress is of crucial importance. 


\section{References}

De Hert, M., Correll, C.U., Cohen, D., 2010. Do antipsychotic medications reduce or increase mortality in schizophrenia? A critical appraisal of the FIN-11 study. Schizophr. Res. 117, 68-74.

Hayes, R.D., Downs, J., Chang, C.K., Jackson, R.G., Shetty, H., Broadbent, M., Hotopf, M., Stewart, R., 2015. The effect of clozapine on premature mortality: an assessment of clinical monitoring and other potential confounders. Schizophr. Bull. 41, 644-55.

Tiihonen, J., Lonnqvist, J., Wahlbeck, K., Klaukka, T., Niskanen, L., Tanskanen, A., Haukka, J., 2009. 11-year follow-up of mortality in patients with schizophrenia: a population-based cohort study (FIN11 study). Lancet 374, 620-7.

Vermeulen, J.M., Van Rooijen, G., Van de Kerkhof, M.P.J., Sutterland, A.L., Correll, C.U., De Haan, L., 2018. Clozapine and Long-Term Mortality Risk in Patients With Schizophrenia: A Systematic Review and Meta-analysis of Studies Lasting 1.1-12.5 Years. Schizophr. Bull. doi: 10.1093/schbul/ sby052

Walker, A.M., Lanza, L.L., Arellano, F., Rothman, K.J., 1997. Mortality in current and former users of clozapine. Epidemiology 8, 671-7.

Wimberley, T., MacCabe, J.H., Laursen, T.M., Sorensen, H.J., Astrup, A., Horsdal, H.T., Gasse, C., Stovring H., 2017. Mortality and Self-Harm in Association With Clozapine in Treatment-Resistant Schizophrenia. Am. J. Psychiatry 174, 990-98. 


\section{Supplementary material}

Supplementary Table 1. Differences in age between users of clozapine and the comparison group in the studies included in the meta-analysis of Vermeulen et al. (2018).

\begin{tabular}{|c|c|c|c|}
\hline \multicolumn{2}{|l|}{ Study } & \multirow[t]{2}{*}{ Clozapine } & \multirow[t]{2}{*}{ Comparison group } \\
\hline Henne: & & & \\
\hline & & Clozapine & Other antipsychotic (haloperidol, risperidone or thioridazine) \\
\hline & Age & $\%$ & $\%$ \\
\hline & $<35$ & 38 & 30 \\
\hline & $35-44$ & 35 & 26 \\
\hline & $45-54$ & 16 & 17 \\
\hline & $55-64$ & 8 & 12 \\
\hline & $65-74$ & 4 & 9 \\
\hline & $>74$ & 1 & 6 \\
\hline \multicolumn{4}{|c|}{ Tiihonen et al. (2009) } \\
\hline & & Clozapine & Any antipsychotic (including clozapine) \\
\hline & Age & OR & OR \\
\hline & $<20$ & 1 & 1 \\
\hline & $20-30$ & 0,66 & 1,25 \\
\hline & $31-40$ & 0,36 & 1,13 \\
\hline & $41-50$ & 0,24 & 0,94 \\
\hline & $51-60$ & 0,15 & 0,86 \\
\hline & $61-70$ & 0,07 & 0,65 \\
\hline & $>70$ & 0,02 & 0,35 \\
\hline
\end{tabular}

Wimberley et al. (2017)

\begin{tabular}{lll} 
& Clozapine & Non clozapine \\
Age & $\%$ & $\%$ \\
$18-29$ & 51,5 & 46,5 \\
$30-56$ & 48,5 & 53,5 \\
\hline
\end{tabular}

Hayes et al. (2015)

\section{Clozapine Non clozapine}

Age, mean $36,7 \quad 43,5$

Kelly et al. (2010)

\section{Clozapine Risperidone}

Age, mean $\quad 39,0 \quad 41,2$

Pridan et al. (2015)

Clozapine Overall

Age, mean 69,4 67,4

In the studies of Taipale et al. (2017) and Modai et al. (2000), no data on age were available. 
Supplementary Table 2. Crude mortality rates, crude mortality rate ratios and adjusted mortality rate ratios in the studies included in the meta-analysis of Vermeulen et al. (2018)

\begin{tabular}{|c|c|c|c|}
\hline Study & Crude MR* & Crude $\mathbf{R R}^{* *}$ & Adj $\mathbf{R R}^{* * *}$ \\
\hline \multicolumn{4}{|l|}{ Hennessy et al. (2002) } \\
\hline Clozapine (reference) & 2,7 & 1 & 1 \\
\hline Haloperidol & 7,3 & 2,70 & 1,25 \\
\hline Risperidone & 7,2 & 2,67 & 1,75 \\
\hline Thioridazine & 6,5 & 2,41 & 1,00 \\
\hline \multicolumn{4}{|l|}{ Taipale et al. (2017) } \\
\hline Fluphenazine LAI\# & 44,8 & 4,04 & 1,57 \\
\hline Flupentixol LAI\# & 19,8 & 1,78 & 0,98 \\
\hline Haloperidol LAI\# & 15,1 & 1,36 & 0,68 \\
\hline Perphenazine LAI\# & 14,0 & 1,26 & 0,70 \\
\hline Zuclopenthixol LAI\# & 16,0 & 1,44 & 0,75 \\
\hline Flupentixol oral & 11,9 & 1,07 & 0,83 \\
\hline Haloperidol oral & 18,3 & 1,65 & 1,11 \\
\hline Levomepromazine oral & 30,8 & 2,77 & 1,45 \\
\hline Perphenazine oral & 9,9 & 0,89 & 0,72 \\
\hline Zuclopenthixol oral & 14,6 & 1,32 & 0,91 \\
\hline Olanzapine LAI\# & 11,8 & 1,06 & 0,74 \\
\hline Paliperidone LAI\# & 4,0 & 0,36 & 0,21 \\
\hline Risperidone LAI\# & 10,0 & 0,90 & 0,58 \\
\hline Aripiprazole oral & 4,3 & 0,39 & 0,42 \\
\hline Clozapine oral (reference) & 11,1 & 1 & 1 \\
\hline Olanzapine oral & 11,4 & 1,03 & 0,91 \\
\hline Quetiapine oral & 12,1 & 1,09 & 0,89 \\
\hline Risperidone oral & 10,3 & 0,93 & 0,77 \\
\hline Other oral & 8,9 & 0,80 & 0,75 \\
\hline Polytherapy & 15,3 & 1,38 & $\mathrm{n} / \mathrm{a}^{+}$ \\
\hline No AP & 21,4 & 1,93 & $\mathrm{n} / \mathrm{a}^{+}$ \\
\hline \multicolumn{4}{|l|}{ Tiihonen et al. (2009) } \\
\hline Clozapine (reference) & 5,69 & 1 & 1 \\
\hline Perphenazine & 10,77 & 1,89 & 1,35 \\
\hline Polypharmacy & 11,19 & 1,97 & 1,46 \\
\hline Olanzapine & 10,5 & 1,85 & 1,53 \\
\hline Thioridazine & 12,32 & 2,17 & 1,54 \\
\hline Risperidone & 15,2 & 2,67 & 1,81 \\
\hline Haloperidol & 19,19 & 3,37 & 1,85 \\
\hline Quetiapine & 16,6 & 2,92 & 1,91 \\
\hline Other & 17,5 & 3,08 & 1,96 \\
\hline
\end{tabular}


Chapter 4

\begin{tabular}{|c|c|c|c|}
\hline & Crude MR* & Crude RR** & Adj $\mathbf{R R}^{* * *}$ \\
\hline \multicolumn{4}{|l|}{ Wimberley et al. (2017) } \\
\hline Clozapine (reference) & 6,0 & 1 & 1 \\
\hline Nonclozapine AP & 8,8 & 1,48 & 1,41 \\
\hline \multicolumn{4}{|l|}{ Hayes et al. (2015) } \\
\hline Clozapine (reference) & 5,6 & 1 & 1 \\
\hline Nonclozapine & 19,3 & 3,45 & 2,5 \\
\hline \multicolumn{4}{|l|}{ Kelly et al. (2010) } \\
\hline Clozapine (reference) & 11,1 & 1 & 1 \\
\hline Risperidone & 9,4 & 0,85 & $\mathrm{n} / \mathrm{a}^{+}$ \\
\hline \multicolumn{4}{|l|}{ Modai et al. (2000) } \\
\hline Clozapine (reference) & 2,7 & 1 & 1 \\
\hline Nonclozapine & 3,2 & 1,20 & $\mathrm{n} / \mathrm{a}^{+}$ \\
\hline \multicolumn{4}{|l|}{ Pridan et al. (2015) } \\
\hline Clozapine (reference) & 41 & 1 & 1 \\
\hline Nonclozapine & 39,4 & 0,96 & $\mathrm{n} / \mathrm{a}^{+}$ \\
\hline
\end{tabular}

* Crude mortality rate per 1000 person year

** Crude mortality rate ratio compared to clozapine

*** Adjusted mortality rate ratio compared to clozapine

${ }^{+}$Not available

\# LAI=long acting injectable 


\section{References}

Hayes, R.D., Downs, J., Chang, C.K., Jackson, R.G., Shetty, H., Broadbent, M., Hotopf, M., Stewart, R., 2015. The effect of clozapine on premature mortality: an assessment of clinical monitoring and other potential confounders. Schizophr. Bull. 41, 644-655.

Hennessy, S., Bilker, W.B., Knauss, J.S., Margolis, D.J., Kimmel, S.E., Reynolds, R.F., Glasser, D.B., Morrison, M.F., Strom, B.L., 2002. Cardiac arrest and ventricular arrhythmia in patients taking antipsychotic drugs: cohort study using administrative data. BMJ. 325(7372), 1070.

Kelly, D.L., McMahon, R.P., Liu, F., Love, R.C., Wehring, H., Shim, J.C., Warren, K.R., Conley, R.R., 2010. Cardiovascular disease mortality in chronic schizophrenia patients treated with clozapine. J. Clin. Psychiatry 71(3), 304.

Modai, I., Hirschmann, S., Rava, A., Kurs, R., Barak, P., Lichtenberg, P., Ritsner, M., 2000. Sudden death in patients receiving clozapine treatment: a preliminary investigation. J. Clin. Psychopharmacol. 20(3), 325-327.

Pridan, S., Swartz, M., Baruch, Y., Tadger, S., Plopski, I., Barak, Y., 2015. Effectiveness and safety of clozapine in elderly patients with chronic resistant schizophrenia. Int. Psychogeriatr. 27(1), 131134.

Taipale, H., Mittendorfer-Rutz, E., Alexanderson, K., Majak, M., Mehtälä, J., Hoti, F., Jedenius, E., Enkusson, D., Leval, A., Sermon, J., Tanskanen, A., Tiihonen, J., 2017. Antipsychotics and mortality in a nationwide cohort of 29,823 patients with schizophrenia. Schizophr. Res. doi: 10.1016/j.schres.2017.12.010

Tiihonen, J., Lonnqvist, J., Wahlbeck, K., Klaukka, T., Niskanen, L., Tanskanen, A., Haukka, J., 2009. 11-year follow-up of mortality in patients with schizophrenia: a population-based cohort study (FIN11 study). Lancet 374, 620-627.

Vermeulen, J.M., Van Rooijen, G., Van de Kerkhof, M.P.J., Sutterland, A.L., Correll, C.U., De Haan, L., 2018. Clozapine and long-term mortality risk in patients with schizophrenia: a systematic review and meta-analysis of studies lasting 1.1-12.5 years. Schizophr. Bull. doi: 10.1093/schbul/sby052

Wimberley, T., MacCabe, J.H., Laursen, T.M., Sorensen, H.J., Astrup, A., Horsdal, H.T., Gasse, C., Stovring H., 2017. Mortality and self-harm in association with clozapine in treatment-resistant schizophrenia. Am. J. Psychiatry 174, 990-998. 



\section{Abstract}

Objectives: Reports of decreased mortality among patients with schizophrenia who use clozapine, may be biased if clozapine is prescribed to relatively healthy patients and if intensive monitoring during its use prevents (under-treatment of) somatic disorder. We aimed to assess whether there is a difference in 1) somatic co-morbidity between patients who start with clozapine and those who start with other antipsychotics, and 2) prescribed somatic medication, between patients using clozapine and those using olanzapine.

Basic methods: Cohort study based on insurance claims (2010-2015). After selecting new users of antipsychotics and those who subsequently switched to clozapine $(\mathrm{N}=158)$, aripiprazole $(\mathrm{N}=295)$, olanzapine $(\mathrm{N}=204)$ or first generation antipsychotics $(\mathrm{N}=295)$, we compared the clozapine starters to others on cardiovascular or diabetic comorbidity. Those using clozapine and olanzapine were compared on new prescriptions for cardiovascular or anti-diabetic drugs.

Main results: The $\mathrm{OR}_{\text {adj }}$ of cardiovascular or diabetic comorbidity among other starters compared to clozapine starters was 0.77 (95\% CI: 0.43-1.39), that is, a non-significantly increased prevalence associated with clozapine was found. Users of clozapine received significantly more new prescriptions for cardiovascular or antidiabetic medication $\left(\mathrm{OR}_{\mathrm{adj}}: 2.70,95 \% \mathrm{CI} 1.43-5.08\right)$.

Conclusion: Starters with clozapine were not cardiovascular/metabolic healthier than starters with other antipsychotics. During its use, they received more somatic treatment.

Key words: schizophrenia, clozapine, epidemiology, outpatients 


\section{Introduction}

Although clozapine is the most effective antipsychotic drug for treatment-resistant schizophrenia, (Siskind et al., 2016, Kane et al., 1988, Souza et al., 2013), it is also a drug with potentially dangerous side-effects like agranulocytosis, myocarditis and ileus (De Berardis et al., 2018). In addition, its use is associated with more metabolic symptoms (e.g. weight gain, adverse effects on lipid profile and type 2 diabetes mellitus (T2DM)) than other antipsychotics except olanzapine, which has a similar profile of metabolic side-effects (Hirsch et al., 2017, Solmi et al., 2017, Leucht et al., 2013).

Surprisingly, a recent meta-analysis (Vermeulen et al., 2018) found a significantly decreased mortality during clozapine use (Mortality rate ratio: 0.56 ; 95\% CI: 0.36-0.85; $\mathrm{p}=.007$ ). However, as we discussed earlier (van der Zalm et al., 2018b), the results of this metaanalysis may be biased because the effect sizes had not been adjusted for age. Nevertheless, some studies (Tiihonen et al., 2009, Hayes et al., 2015) using age-adjusted effect measures reported a decreased mortality during the use of clozapine compared to the use of other antipsychotics. Considering that schizophrenia shortens life expectancy by more than 20 years (Laursen et al., 2014), it is important to investigate whether clozapine has the potential to increase or decrease this mortality gap and what the mechanism of action is.

A possible explanation for findings of lower mortality during clozapine use is confounding by indication: given its adverse side-effects, physicians may be reluctant to prescribe this drug to patients who have already been diagnosed with (or are at high risk for) cardiovascular disorder or diabetes mellitus. Another explanation is that the intensive monitoring required during the use of clozapine, leads to a more adequate treatment of risk factors for these disorders. For example, weight gain, high blood pressure and increased glucose may be noted and treated more often than during the use of other antipsychotics that do not require weekly or monthly checks. As patients with schizophrenia are in general somatically undertreated (Laursen et al., 2011, Swildens et al., 2016), clozapine monitoring may result in better somatic care and thus to a decreased mortality (Kugathasan et al., 2018).

We therefore aimed 1) to compare cardiovascular and diabetics comorbidity (diagnoses and pertinent medication) between new users of antipsychotics who, after a period of use of a non-clozapine antipsychotic, switch to clozapine, to their counterparts who, after a similar period switch to olanzapine, aripiprazole or First Generation Antipsychotics (FGAs); 2) to determine whether patients are more likely to receive new cardiovascular or anti-diabetic drugs during the use of clozapine than during the use of olanzapine. Since olanzapine has similar metabolic effects as clozapine, but does not require weekly or monthly check-ups, it is the comparison drug for question 2 . 


\section{Methods}

\section{Data source}

For this retrospective cohort study, data from the Achmea Health Insurance Database (AHID) was used. This database of healthcare claims covers over 4 million subjects in the Netherlands, about $25 \%$ of the population. Insurance for the provision of medical care is compulsory for all Dutch citizens. Health insurance companies are legally obliged to provide citizens with insurance. Therefore, the AHID may be regarded as highly representative for the health care utilization of the Dutch population. Besides information on patients characteristics (age and sex), the AHID contains records of every diagnostic and therapeutic provision (so called diagnosis-treatment-combinations). In the list of all 4,391 possible somatic diagnosis-treatment-combinations used by insurance companies the cardiovascular or diabetic diagnoses were identified by authors FT and YZ independently and differences were discussed with author JPS to reach consensus. The AHID also includes information on all reimbursed drugs. Drug prescriptions during hospitalizations are not registered in this database.

\section{Study cohort and procedures}

The first data-set consisted of all prescriptions of antipsychotic drugs, Anatomical Therapeutic Chemical (ATC) classification code N05A, between January 1st 2010 and January 1st 2016, to patients aged at least 18 years. First, the records for lithium, pipamperon, levomepromazine, periciazine, droperidol, and tiapride were removed from this data-set, because in the Netherlands these drugs are mostly prescribed for other indications than schizophrenia or other related psychotic disorders. Second, this data-base was linked to a data-base with all insured subjects and we identified all patients who used clozapine, aripiprazole, olanzapine and FGAs during the observation period. We selected these antipsychotics for their similar (olanzapine) or different (aripiprazole and FGA's) profile of metabolic side-effects compared to clozapine. Third, in an attempt to select patients with a first episode of psychosis, we restricted our analyses to "new users" of antipsychotics. For this purpose we selected those who started an antipsychotic after at least one year of no use (a longer period was not desirable since prescribing data of only six years were available). Some of them may have been neuroleptic-naive, others may have interrupted their ambulatory treatment for a year or may have been hospitalized for a year (medication use is not registered during hospitalization). Fourth, within this group of new users, we identified patients who were switched to clozapine, aripiprazole, olanzapine and FGAs, after at least 6 months use of non-clozapine antipsychotics. This restriction was necessary because clozapine is a third-line treatment and therefore normally not the drug to start with. In practice, it usually takes at least 6 months to find out that the patient is treatment-resistant. Fifth, patients with records for drugs for Parkinson's Disease (ATC code N04B) or dementia 
(ATC code N06D) were removed from the cohort, because antipsychotics are regularly prescribed to patients with Parkinson's Disease and dementia. Sixth, this data-base was linked to the data-base with the somatic diagnosis-treatment-combinations. Patients with a diagnosis of Parkinson's disease or dementia were removed. At last, in order to select only patients with a diagnosis of schizophrenia or other related psychotic disorder, the drug prescription file was linked to a data-base with codes for psychiatric diagnosistreatment-combinations (17 codes for different psychiatric disorders).

\section{Statistical analysis}

Descriptive statistics were used to assess baseline characteristics. Clozapine-users were compared to users of olanzapine, aripiprazole and FGAs with regard to age (t-test) and sex $(\chi 2$ test). To compare cardiovascular and diabetic comorbidity, between patients who start with clozapine and those who start with olanzapine, aripiprazole or FGAs, we performed a logistic regression, adjusting for age and sex. The independent variable was start with clozapine, olanzapine, aripiprazole or FGAs with clozapine as a reference category. The dependent variable was any diagnosis of a cardiovascular disorder or diabetes mellitus type, I or II, or a drug prescribed for these disorders in the four months preceding the start of this antipsychotic drug (yes/no). Our restriction to four months was to ensure that the somatic comorbidity was present and could not be overlooked by the prescribing physician at the moment of starting an antipsychotic. Cardiovascular comorbidity was defined as treatment related to a cardiovascular diagnosis or the use of cardiovascular drugs: ATC codes B01AA, C01, CO2, CO3, C07, C08, C09, C10 (see supplementary Table $\mathrm{S} 1$ ). Diabetic comorbidity was defined as treatment related to a diagnosis of diabetes mellitus or the use of drugs for diabetes: ATC code A10. In addition, we conducted a similar univariate analysis, with olanzapine, aripiprazole and FGAs combined.

For the second question, as to whether users of clozapine are more likely to receive a prescription for cardiovascular or anti-diabetic drugs than users of olanzapine, a cox regression analysis was used. The new users of clozapine and olanzapine were followed (during the use of these drugs) and compared on new use (i.e. no use in the four months preceding the particular antipsychotic) of (1) cardiovascular drugs and (2) ant-diabetic drugs adjusting for age and sex. The follow-up started at the day of the first prescription of either clozapine or olanzapine and ended at the day of the last prescription. An additional logistic regression was performed to assess for differences in proportions of patients who started with cardiovascular or anti-diabetic drugs, regardless of the duration of their follow-up period. The cox regression and logistic regression analyses were also performed for all cardiovascular drugs separately. The statistics were performed with SPPS, version 22.0. A two-tailed p-value of $<0.05$ was considered statistically significant for all tests. 


\section{Ethical approval}

The Executive Board of Achmea gave permission to use their data for this study. Since data had been anonymized, ethical approval was not required.

\section{Results}

\section{Description of cohort}

In the 6-year observation period, 84,156 persons were using antipsychotic drugs. See Figure 1 for a flow diagram. After elimination of those who did not meet the requirement of no antipsychotic use during at least one year and those who were not switched to another antipsychotic after at least 6 months of use, the study population consisted of 952 persons switching to clozapine $(\mathrm{N}=158)$, aripiprazole (295), olanzapine (204) or first generation antipsychotics (295). Their baseline characteristics are shown in Table 1. Given their mean age of over 40 years old, it is unlikely that the cohort only consisted of neuroleptic-naïve patients.

Table 1. Baseline characteristics of 952 patients switching to clozapine, aripiprazole, olanzapine or first generation antipsychotics after 1 year of no use of antipsychotics and at least 6 months of other antipsychotic use.

\begin{tabular}{lrrrr}
\hline & Clozapine & Aripiprazol & Olanzapine & $\begin{array}{r}\text { First generation } \\
\text { antipsychotics } \\
\text { N=295 }\end{array}$ \\
\hline Age, mean (SD) & $\mathbf{N = 1 5 8}$ & $\mathbf{N = 2 9 5}$ & $\mathbf{N = 2 0 4}$ & $\begin{array}{r}\text { (5) } \\
\text { Sex, male (\%) }\end{array}$ \\
\hline $1.7(15.8)$ & $42.5(13.1)$ & $48.9(16.9)$ & $43.7(15.0)$ \\
Diabetes Mellitus diagnosis (\%) & $111(70.3)$ & $170(57.6)$ & $122(59.8)$ & $182(61.7)$ \\
Cardiovascular diagnosis (\%) & $1(0.6)$ & $5(1.7)$ & $3(1.5)$ & $9(3.1)$ \\
$\begin{array}{l}\text { Drugs for diabetes, without } \\
\text { diagnosis (\%) }\end{array}$ & $11(7.0)$ & $12(4.1)$ & $17(8.3)$ & $11(3.7)$ \\
$\begin{array}{l}\text { Cardiovascular drugs, without } \\
\text { diagnosis (\%) }\end{array}$ & 0 & 0 & 0 & $2(0.7)$ \\
\hline
\end{tabular}




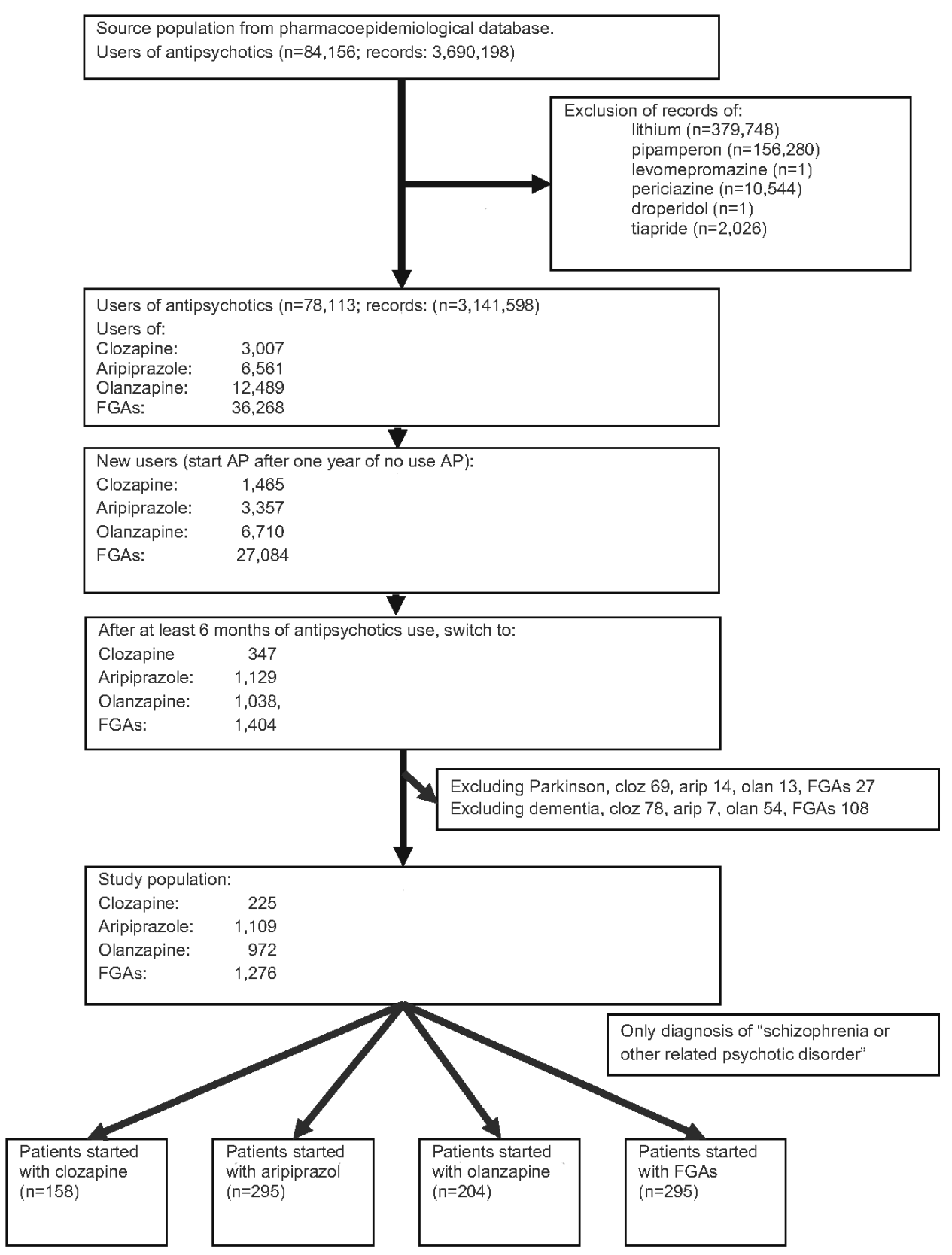

Figure 1 Flowchart 


\section{Cardiovascular and diabetic comorbidity at start of antipsychotic treatment}

The results of the logistic regression are shown in Table 2. There were differences in age and sex between the clozapine starters and starters with other antipsychotics in that the olanzapine starters were significantly older than the clozapine starters $(\mathrm{p}<.001)$ and that there were significantly more female aripiprazole starters compared to clozapine starters $(\mathrm{p}=.009)$. After adjusting for age and sex, starters with other antipsychotics had less cardiovascular or diabetic comorbidity than starters with clozapine, although the difference was not statistically significant (Odds Ratio [OR]: 0.77; 95\% CI: 0.43-1.39; $\mathrm{p}=.388)$. The difference between olanzapine-starters and clozapine-starters was smallest $\left(\mathrm{OR}_{\text {adj: }} 0.92 ; 95 \%\right.$ CI: 0.46-1.84; $\left.\mathrm{p}=.818\right)$.

Table 2. Results of a logistic regression, comparing starters with aripiprazole, olanzapine and first generation antipsychotics to starters with clozapine (reference) on cardiovascular and diabetic comorbidity.

\begin{tabular}{lrrrrrr}
\hline & Crude OR & 95\% CI & $\boldsymbol{p}$ & Adj. OR* & 95\% CI & $\boldsymbol{p}$ \\
\hline Aripiprazol & 0.58 & $0.29-1.17$ & .125 & 0.58 & $0.28-1.19$ & .138 \\
Olanzapine & 1.18 & $0.61-2.31$ & .622 & 0.92 & $0.46-1.84$ & .818 \\
First generation antipsychotics (FGAs) & 0.89 & $0.47-1.71$ & .736 & 0.85 & $0.44-1.64$ & .619 \\
$\begin{array}{l}\text { Aripiprazol, olanzapine and FGAs } \\
\text { combined }\end{array}$ & 0.85 & $0.48-1.50$ & .563 & 0.77 & $0.43-1.39$ & .388 \\
\hline
\end{tabular}

* Adjusted for age and sex

\section{Cardiovascular and anti-diabetic drugs during use of olanzapine and clozapine}

From the 158 starters with clozapine, 13 had a single prescription for such a drug. They were excluded because (as a result of our definition) their duration of follow-up was 0 days. The mean duration of clozapine use for the remaining 145 patients was 487 days (SD 425). From the 204 starters with olanzapine, 30 had a single prescription and were excluded. The remaining 174 patients used olanzapine with a mean duration of 422 days (SD 408). Table 3 shows the results of the Cox regression analysis, adjusting for age and sex. Physicians prescribed significantly earlier cardiovascular drugs (Hazard ratio [HR]: $2.31 ; 95 \%$ CI $1.44-3.69 ; \mathrm{p}<.001)$ to users of clozapine than to users of olanzapine. There was a trend in prescribing anti-diabetic drugs to users of clozapine earlier (HR: 2.53; 95\%CI: 1.00-6.39; $\mathrm{p}=.051$ ). The results of the logistic regression in Table 3, show that cardiovascular or antidiabetic drugs were not only prescribed in an earlier stage, but also more often. Supplementary Table S2 shows that adjusting for cardiovascular/diabetic diagnoses or drugs before the start with clozapine or olanzapine does not change the results. 
Table 3 Results of a cox regression and a logistic regression comparing starters with clozapine to starters with olanzapine on new cardiovascular and diabetic drugs during the use of clozapine or olanzapine.

\begin{tabular}{lrrrrrrrr}
\hline Cox regression & $\begin{array}{r}\text { Clozapine } \\
\mathbf{N = 1 4 5}\end{array}$ & $\begin{array}{r}\text { Olanzapine } \\
\mathbf{N = 1 7 4}\end{array}$ & HR $^{*}$ & $\mathbf{9 5 \%} \mathbf{C I}$ & $\boldsymbol{p}$ & $\mathbf{O R}^{*}$ & $\mathbf{9 5 \%} \mathbf{C I}$ & $\boldsymbol{p}$ \\
\hline Anti-diabetes drugs & 12 & 8 & 2.53 & $1.00-6.39$ & .051 & 2.51 & $0.96-6.60$ & .062 \\
$\mathrm{~N}=20$ & & & & & & & & \\
Cardiovascular drugs & 37 & 39 & 2.31 & $1.44-3.69$ & $<.001$ & 2.73 & $1.41-5.27$ & .003 \\
$\mathrm{~N}=76$ & & & & & & & & \\
$\quad \begin{array}{l}\text { Total } \\
\mathrm{N}=81\end{array}$ & 40 & 41 & 2.38 & $1.51-3.76$ & $<.001$ & 2.70 & $1.43-5.08$ & .002 \\
\hline
\end{tabular}

* Adjusted for age and sex

The differences in cardiovascular drugs were most prominent for 'lipid modifying agents' and 'beta blocking agents', see supplementary Table S1. However, after correcting for multiple testing, only 'beta blocking agents' were prescribed significantly earlier to users of clozapine.

\section{Discussion}

\section{Main findings}

The objective of this study was to examine whether patients who started with clozapine were relatively healthier at the start and better somatically monitored during its use, compared to patients who started with other antipsychotics. The results showed that users of clozapine were not having less cardiovascular or diabetic comorbidity. On the contrary, they seemed to have more cardiovascular or diabetic comorbidity. These findings suggest that confounding by indication is not a likely explanation for lower mortality in clozapine users. However, during the use of clozapine new cardiovascular or antidiabetic drugs were prescribed earlier and more often, compared to those using olanzapine. Although clozapine-users were having more cardiovascular or diabetic diagnoses (8\%), this difference is small and does not explain the large difference $(H R>2)$ in prescribed drugs during the use of clozapine found in this study, see supplementary table 2.

\section{Interpretation and comparison to other studies}

Our observation that clozapine is not prescribed to relatively healthy patients contradicts findings made in Canada (Vanasse et al., 2016), but not a report from Denmark (Wimberley et al., 2017). Vanasse et al. reported that starters with clozapine in Canada were physically healthier compared to starters with other antipsychotics (only 10.8\% had a comorbidity index of 1 or higher, compared to $23.9 \%$ for users of other antipsychotics. Wimberley et al., however, using the same comorbidity index, found a proportion of 
$16.3 \%$ among the clozapine starters with a score of 1 or higher, which was not very different from starters with other antipsychotics (17.3\%). The differences in prescription rates between countries may result in differences in cautiousness regarding prescribing clozapine. To illustrate this, the prescription rates in Canada were very low $(1.7 \%)$, whereas in Denmark 57.9\% of the treatment-resistant patients was using clozapine. Assuming the prevalence of treatment resistance is about 30\%, the prescription rate in Denmark is about 15\%. In the Netherlands prescription rates are relatively high (21.6\%) (van der Zalm et al., 2018a), which may explain the less restrictive use in patients with somatic comorbidity.

There are as yet no studies on the effect of better somatic monitoring in patients using clozapine. Hayes et al. (Hayes et al., 2015) reported that those prescribed clozapine had more face-to-face clinical contact compared to those not prescribed clozapine, but they did not provide information on the prescription of somatic drugs.

The differences between users of clozapine and users of olanzapine in somatic treatment in our study were most prominent in the following categories of drugs: 'drugs for diabetes', 'beta blocking agents' and 'lipid modifying agents' (statins).

Drugs for diabetes are prescribed in (the beginning of) diabetes, but are also effective to prevent weight gain (de Silva et al., 2016). As shown by Leucht (Leucht et al., 2013) olanzapine causes more weight gain than any other antipsychotic, but the difference with clozapine was non-significant. Studies have also failed to show a significant difference in the risk for T2DM between users of clozapine and users of olanzapine, (Hirsch et al., 2017, Solmi et al., 2017, Komossa et al., 2010, Jesus et al., 2015). Therefore, the difference in prescription of anti-diabetic drugs is not likely to be explained by differences in risk of weight gain or T2DM.

Beta blocking agents are mostly prescribed for high blood pressure. Clozapine and olanzapine can cause both hypotension and hypertension and no differences between these 2 agents have been found in this respect (Kelly et al., 2014). However, clozapine is also known to cause tachycardia (Miller, 2000), especially in the initiation phase. The difference between clozapine and olanzapine in prescriptions of beta blocking agents may therefore be caused to a certain extent by the higher risk of tachycardia associated with the use of clozapine.

Previous studies have shown that the proportions of patients with high LDL cholesterol and triglycerides rates among users of olanzapine and clozapine are similar (30-40\%) (Birkenaes et al., 2008). Our result of lipid modifying agents in $12.6 \%$ of olanzapineusers and $14.5 \%$ for clozapine-users, confirms the undertreatment earlier mentioned. 
The more than two-fold increased odds of receiving lipid modifying agents among users of clozapine emerged after adjustment for age.

\section{Strengths and limitations}

To our knowledge, this is the first study to investigate whether patients using clozapine receive better physical health care. A strength of this study is the large representative database consisting of almost a fourth of the Dutch population.

There are limitations that need to be addressed. First, since the register did not provide DSM-IV or ICD-diagnoses, but so-called diagnosis-treatment-combinations, we could only use the less specific term 'schizophrenia or other related psychotic disorders'. Second, BMI, blood glucose and dose of prescribed antipsychotic were not available, making it impossible to compare number and severity of metabolic side-effects between different groups. Third, other important variables like duration of illness or duration of untreated illness were not available too. Fourth, reasons for prescribing clozapine were unknown. We only included patients with a diagnosis of schizophrenia or other related psychotic disorder, but there might have been patients for whom the diagnosis was reconsidered and changed into bipolar disorder at a later stage. Finally, an unknown proportion of the "new users" of antipsychotics in our study were not neuroleptic-naive. A part of them may have used antipsychotics in the past and interrupted their ambulatory treatment for more than a year. Another small part of these "new users" may have started outpatient antipsychotic use after at least a year of in-patient stay (which is not recorded in the data-base). However, to answer the questions in our study, patients do not necessarily have to be neuroleptic-naive.

\section{Conclusion}

We found that clozapine is not prescribed to relatively healthy patients. The more frequent prescription of cardiovascular and anti-diabetic drugs to patients using clozapine (vs. olanzapine), may be the result of more adequate somatic treatment. This is an important finding as it suggests that regular check-ups for psychotic patients may improve somatic care and could help decrease mortality. 


\section{References}

Birkenaes AB, Birkeland KI, Engh JA, Faerden A, Jonsdottir H, Ringen PA, et al. (2008). Dyslipidemia independent of body mass in antipsychotic-treated patients under real-life conditions. $J$ Clin Psychopharmacol 28:132-7.

De Berardis D, Rapini G, Olivieri L, Di Nicola D, Tomasetti C, Valchera A, et al. (2018). Safety of antipsychotics for the treatment of schizophrenia: a focus on the adverse effects of clozapine. Ther Adv Drug Saf 9:237-256.

de Silva V A, Suraweera C, Ratnatunga SS, Dayabandara M, Wanniarachchi N, Hanwella R. (2016). Metformin in prevention and treatment of antipsychotic induced weight gain: a systematic review and meta-analysis. BMC Psychiatry 16:341.

Hayes RD, Downs J, Chang CK, Jackson RG, Shetty H, Broadbent M, et al. (2015). The effect of clozapine on premature mortality: an assessment of clinical monitoring and other potential confounders. Schizophr Bull 41:644-655.

Hirsch L, Yang J, Bresee L, Jette N, Patten S, Pringsheim T. (2017). Second-Generation Antipsychotics and Metabolic Side Effects: A Systematic Review of Population-Based Studies. Drug Saf 40:771-781.

Jesus C, Jesus I, Agius M. (2015). What evidence is there to show which antipsychotics are more diabetogenic than others? Psychiatr Danub 27:S423-428.

Kane J, Honigfeld G, Singer J, Meltzer H. (1988). Clozapine for the treatment-resistant schizophrenic. A double-blind comparison with chlorpromazine. Arch Gen Psychiatry 45:789-796.

Kelly AC, Sheitman BB, Hamer RM, Rhyne DC, Reed RM, Graham KA, et al. (2014). A naturalistic comparison of the long-term metabolic adverse effects of clozapine versus other antipsychotics for patients with psychotic illnesses. J Clin Psychopharmacol 34:441-445.

Komossa K, Rummel-Kluge C, Hunger H, Schmid F, Schwarz S, Duggan L, et al (2010). Olanzapine versus other atypical antipsychotics for schizophrenia. Cochrane Database Syst Rev(3), Cd006654. doi:10.1002/14651858.CD006654.pub2

Kugathasan P, Horsdal HT, Aagaard J, Jensen SE, Laursen TM, Nielsen RE. (2018). Association of Secondary Preventive Cardiovascular Treatment After Myocardial Infarction With Mortality Among Patients With Schizophrenia. JAMA Psychiatry 75:1234-1240.

Laursen TM, Munk-Olsen T, Gasse C. (2011). Chronic somatic comorbidity and excess mortality due to natural causes in persons with schizophrenia or bipolar affective disorder. PLoS One 6:(9), e24597. doi:10.1371/journal.pone.0024597

Laursen TM, Nordentoft M, Mortensen PB (2014). Excess early mortality in schizophrenia. Annu Rev Clin Psychol 10:425-448.

Leucht S, Cipriani A, Spineli L, Mavridis D, Orey D, Richter F, et al (2013). Comparative efficacy and tolerability of 15 antipsychotic drugs in schizophrenia: a multiple-treatments meta-analysis. Lancet 382:951-962.

Miller DD. (2000). Review and management of clozapine side effects. J Clin Psychiatry 61 Suppl 8, 14-17; discussion 18-19.

Siskind D, McCartney L, Goldschlager R, Kisely S. (2016). Clozapine v. first- and second-generation 
antipsychotics in treatment-refractory schizophrenia: systematic review and meta-analysis. $\mathrm{Br} J$ Psychiatry 209:385-392.

Solmi M, Murru A, Pacchiarotti I, Undurraga J, Veronese N, Fornaro M, et al. (2017). Safety, tolerability, and risks associated with first- and second-generation antipsychotics: a state-of-the-art clinical review. Ther Clin Risk Manag 13:757-777.

Souza JS, Kayo M, Tassell I, Martins CB, Elkis H. (2013). Efficacy of olanzapine in comparison with clozapine for treatment-resistant schizophrenia: evidence from a systematic review and metaanalyses. CNS Spectr 18:82-89.

Swildens W, Termorshuizen F, de Ridder A, Smeets H, Engelhard IM. (2016). Somatic Care with a Psychotic Disorder. Lower Somatic Health Care Utilization of Patients with a Psychotic Disorder Compared to Other Patient Groups and to Controls Without a Psychiatric Diagnosis. Adm Policy Ment Health 43:650-662.

Tiihonen J, Lonnqvist J, Wahlbeck K, Klaukka T, Niskanen L, Tanskanen A, et al. (2009). 11-year followup of mortality in patients with schizophrenia: a population-based cohort study (FIN11 study). Lancet 374:620-627.

van der Zalm YC, Termorshuizen F, Schulte PF, Bogers JP, Marcelis M, Sommer IE, et al. (2018a). Prescription and Underprescription of Clozapine in Dutch Ambulatory Care. Front Psychiatry 9: 231.

van der Zalm YC, Termorshuizen F, Selten JP. (2018b). Concerns about bias in studies on clozapine and mortality. Schizophr Res 204:425-426.

Vanasse A, Blais L, Courteau J, Cohen AA, Roberge P, Larouche A, et al. (2016). Comparative effectiveness and safety of antipsychotic drugs in schizophrenia treatment: a real-world observational study. Acta Psychiatr Scand 134:374-384.

Vermeulen JM, van Rooijen G, van de Kerkhof MPJ, Sutterland AL, Correll CU, de Haan L. (2018). Clozapine and Long-Term Mortality Risk in Patients With Schizophrenia: A Systematic Review and Meta-analysis of Studies Lasting 1.1-12.5 Years. Schizophr Bull 45:315-329.

Wimberley T, MacCabe JH, Laursen TM, Sorensen HJ, Astrup A, Horsdal HT, et al. (2017). Mortality and Self-Harm in Association With Clozapine in Treatment-Resistant Schizophrenia. Am J Psychiatry 174:990-998. 


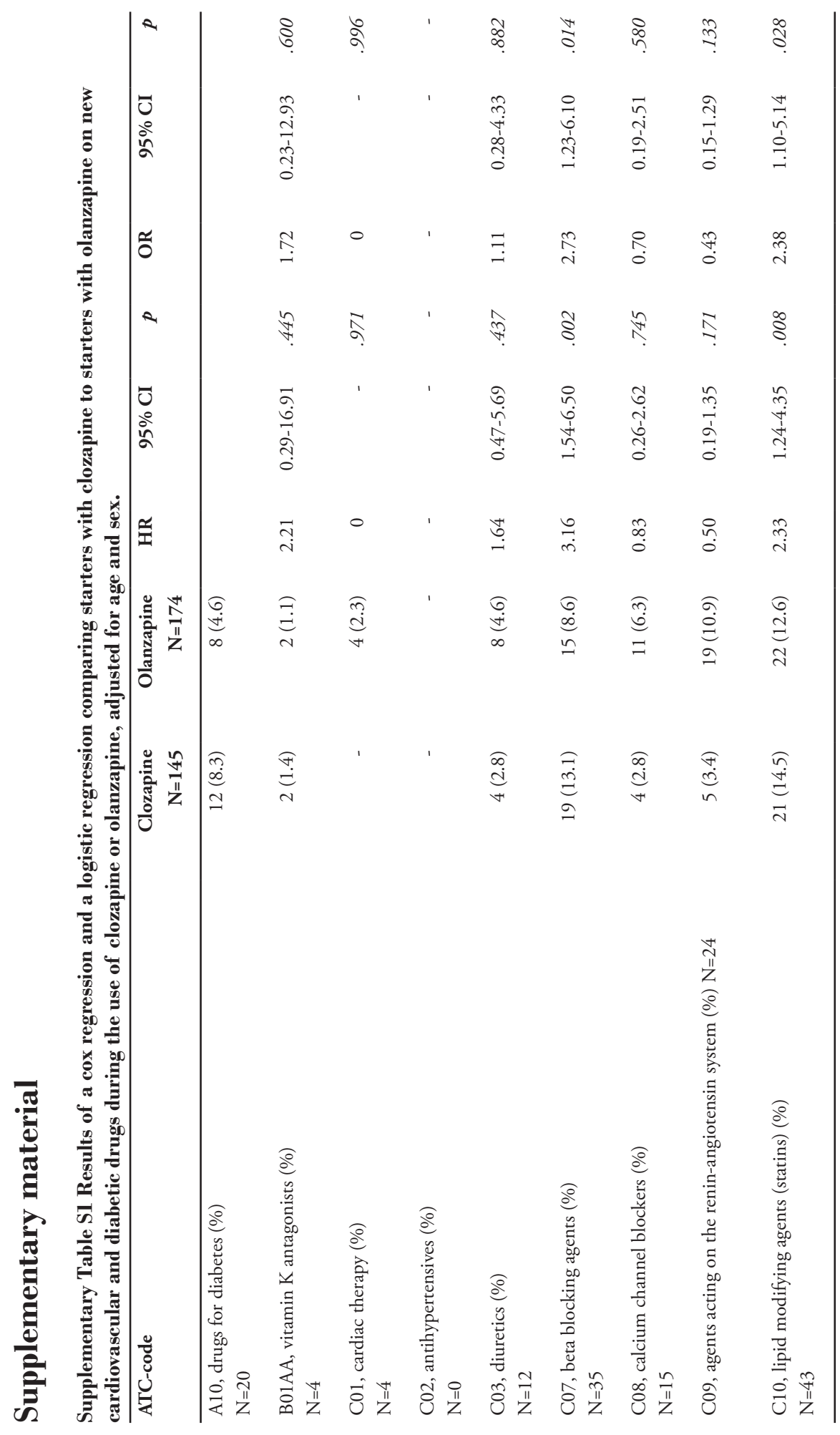




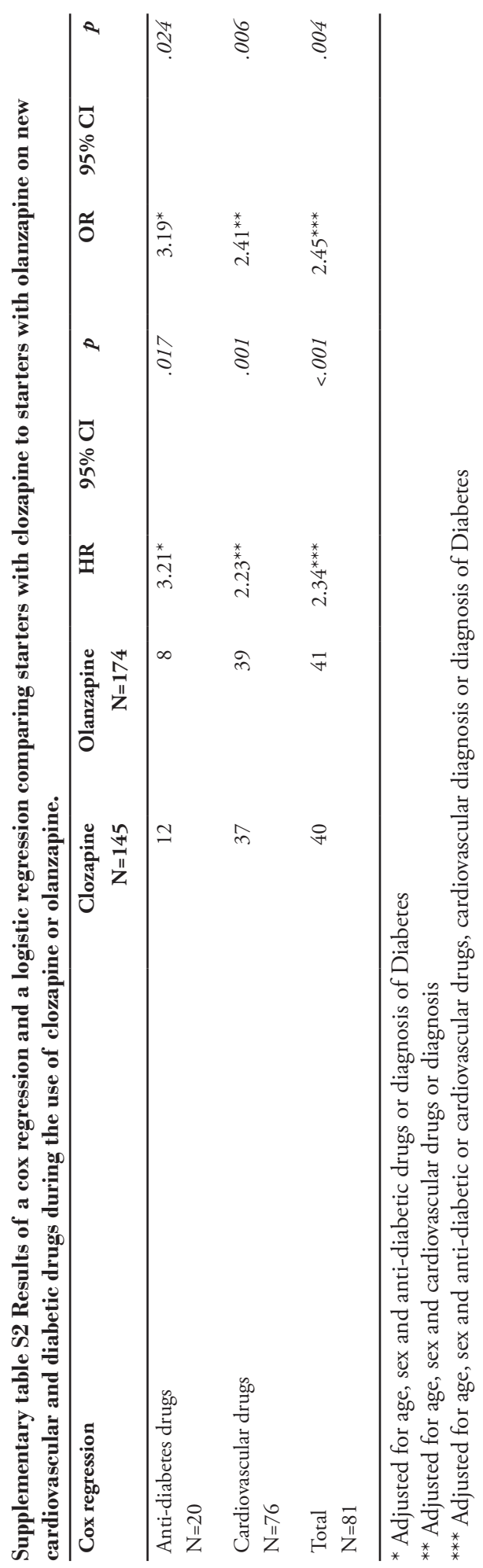





\section{Abstract}

Objective: To compare the mortality in people using clozapine to that of people using other antipsychotics.

Methods: Danish incidence cohort of 22110 patients with a first diagnosis of non-affective psychotic disorder (1995-2013) and a prevalence cohort of 50881 patients ever diagnosed with such a disorder (1969-2013). Hazard ratios (HR) were calculated for the antipsychotic drug used at the time of death ('current use': incidence and prevalence cohort), and for the drug used for the longest at that moment ('cumulative use': incidence cohort), using a Cox model with adjustment for somatic comorbidity. Clozapine was the reference drug.

Results: As for current drug use, the risk of suicide was higher among users of other antipsychotics in the incidence $\left(\mathrm{HR}_{\mathrm{adj}}=1.76 ; 95 \% \mathrm{CI} 0.72-4.32\right)$ and prevalence $\left(\mathrm{HR}_{\mathrm{adj}}=2.20 ; 95 \% \mathrm{CI} 1.35-3.59\right)$ cohorts. There was no significant difference in allcause or cardiovascular mortality in the two cohorts. Cumulative use of clozapine was not associated with an increased cardiovascular mortality. Cumulative use of other antipsychotics for up to 1 year was associated with a lower all-cause mortality and suicide risk than a similar period of clozapine use (all-cause: $\mathrm{HR}_{\mathrm{adj}}=0.73 ; 95 \%$ CI 0.63-0.85, suicide; $\mathrm{HR}_{\mathrm{adj}}=0.65 ; 95 \%$ CI $0.46-0.91$ ).

Conclusion: The results indicate that the use of clozapine is not associated with increased cardiovascular mortality. We found opposing trends towards a lower risk of suicide during current use of clozapine and a higher risk of suicide associated with cumulative use up to 1 year. This suggests that clozapine cessation marks a period of high risk of suicide.

Keywords: psychosis, clozapine, mortality, outpatient treatment 


\section{Significant outcomes}

- Despite the long-term follow-up, we found no major differences in cardio-vascular mortality between users of clozapine and users of other antipsychotics.

- The findings add to an increasing body of evidence that clozapine use is associated with a reduced risk of suicide.

- Clinicians should carefully monitor patients from whom clozapine has been withdrawn within the first year, because these patients are at an increased risk of suicide.

\section{Limitations}

- Information was available on dispensing of drugs, but not on actual use of drugs.

- Drug use during hospital admission was unknown.

- The analysis of cumulative use included only the drug that had been used for the longest time at the time of death.

\section{Data availability statement}

The data are not publicly available due to privacy or ethical restrictions. 


\section{Introduction}

Clozapine is an effective drug for treatment-resistant schizophrenia, but has some serious side-effects such as agranulocytosis, myocarditis, and ileus (De Berardis et al., 2018). Since it is also associated with more metabolic side-effects than most other antipsychotics (Hirsch et al., 2017), long-term use could have a negative impact on life expectancy. Nevertheless, some studies have reported a significant reduction in allcause mortality and mortality due to suicide (Hayes et al., 2015; Tiihonen et al., 2009; Vermeulen et al., 2019; Walker, Lanza, Arellano, \& Rothman, 1997). The methodology of these studies has been commented (De Hert, Correll, \& Cohen, 2010; van der Zalm, Termorshuizen, \& Selten, 2019). An example is survivor bias, an important source of confounding when studying clozapine and suicide. The risk of suicide is highest in the first years after illness onset (Termorshuizen et al., 2013), whereas clozapine is often first prescribed later (Howes et al., 2012). It is difficult to address this confounding effect of time since diagnosis in a prevalence cohort, because a number of patients will have been diagnosed at an unknown point in time before the start of the follow-up. A single study used an incidence cohort (Kiviniemi, 2013), but it did not address survivor bias, because the results were not adjusted for duration since onset of illness.

A long observation period is required to study the association between clozapine and cardiovascular mortality and it is essential to distinguish between the drug used at the time of death ("current use") and the long-term use of a drug (often designated "cumulative use"), since patients on clozapine may be switched to another antipsychotic shortly before death. It is also important to adjust the results for somatic co-morbidity or treatment, because clinicians may prescribe clozapine more often to patients in better cardiovascular health and because mandatory monitoring may lead to more adequate treatment of somatic disorder. To our knowledge, only two studies on cardiovascular mortality used a measure of cumulative use (Taipale et al., 2020; Tiihonen et al., 2009). The results of these studies showed no differences between cumulative use of clozapine and cumulative use of other antipsychotics, but the results were not adjusted for somatic co-morbidity and the treatment thereof.

\section{Aims of the study}

In view of the limitations of the above mentioned studies, the aim of the present study was to critically re-assess the association between clozapine and mortality. We investigated, in a nationwide Danish cohort, whether mortality (all-cause, due to suicide, or due to cardiovascular disease) associated with current use or cumulative use of clozapine is lower than that associated with current use or cumulative use of other categories of antipsychotics, adjusting for somatic comorbidity and the treatment thereof. 


\section{Materials and methods}

More details on material and methods are given in Appendix 1.

\section{Data sources}

In this multi-register Danish cohort study, the following databases were linked: (1) The Danish Psychiatric Central Research Register. The computerized registration started on 1 April 1969 and all outpatient contacts were registered after 1994; (2) The Danish National Prescription Registry for information on dispensed drugs for all Danish inhabitants, from 1995 onwards; (3) The Causes of Death Register from 1970 to 2015; (4) The Danish National Patient Registry for somatic health care records from 1977 to 2015; (5) The Danish Civil Registration System from 1973 to 2015. The data that support the findings of this study are available on request from the corresponding author.

\section{Subjects}

We defined an incidence cohort, the members of which were all Danish inhabitants aged 15-100 years who were diagnosed with a first non-affective psychotic disorder (NAPD: ICD-8 295 and 299; ICD-10 F20, F25, F28 and F29) between 1 January 1995 and 30 June 2013. Migrants to Denmark were excluded, because information on diagnosis and drug use in the period before immigration was not available. We also defined a prevalence cohort with all individuals ever diagnosed with an NAPD up to 30 June 2013. This cohort included all members of the incidence cohort, plus patients with a first-registered diagnosis before 1 January 1995 and migrants.

\section{Outcomes}

The primary outcome was all-cause mortality. Secondary outcomes were suicide and cardiovascular mortality.

\section{Exposure}

Treatment was categorized as follows: 1) clozapine (reference); 2) olanzapine; 3) risperidone; 4) other Second-Generation Antipsychotics (SGAs); 5) First Generation Antipsychotics (FGAs); 6) polypharmacy including clozapine; 7) polypharmacy not including clozapine; 8) no antipsychotic medication; 9) hospital-delivered antipsychotic, type unknown: antipsychotics are distributed free of charge to patients sentenced to treatment and, since 2008, during the first 2 years subsequent to a diagnosis of schizophrenia. The type of antipsychotic is not known because the drug is not registered in the prescription registry. A small proportion of these patients will not be using any antipsychotic; 10) Drug Unknown: no data available because of hospitalization, inpatient drug use is not registered in the prescription registry. Episodes of drug use were censored on day 15 of hospitalization. We chose this time period 
because antipsychotic drugs are often continued at the start of a hospitalization and because their effects are likely to last during this period; 11) no use of antipsychotics. We conducted separate analyses for current and cumulative use of antipsychotics. Both current and cumulative use are time-dependent variables and were recalculated at the time of each death event in the cohort, both for the patient who died and for those who were still alive at that time. The currently used antipsychotic was defined as the last drug that was prescribed before a death in the cohort, provided that death occurred after no more than 2 weeks of no use or no more than 2 weeks after hospital admission. Cumulative use was defined as a time-dependent variable as well and was recalculated at the time of each death in the cohort. For this measure, all episodes of use of a certain antipsychotic were aggregated and the total duration of these episodes was categorized as follows: $0-1,1-3,3-6,6-10$ years, and more than 10 years. Thus, one individual could contribute to several monotherapy or polypharmacy categories at different points in time during follow-up. However, when a death occurred, a subject was placed in only one category of cumulative antipsychotic use, namely, in the category of the drug that had been used the longest at that time. This implies that shorter periods of use of other antipsychotics at this point in time were disregarded. To illustrate this, after consecutively 2 years of olanzapine, 4 years of clozapine and 3 months of risperidone use, the patient is in the category "risperidone" for the analysis of current use and in the category "clozapine (3-6years)" for the cumulative use analysis. After 3 months of risperidone, 9 months of olanzapine and 6 months of clozapine use, a patient is in the "clozapine" category for the analysis of current use and in the category "olanzapine (0-1 year)" for the analysis of cumulative use. Hazard ratios were calculated with the category clozapine use as reference.

\section{Covariates}

Baseline variables were age at start of follow-up, sex, primary psychiatric diagnosis, and psychiatric hospitalization before follow-up (yes/no). We included the latter variable as a measure of the severity of illness. Duration of illness, i.e., duration since first registered diagnosis of NAPD at the time of cohort entry, was another baseline variable for members of the prevalence cohort. Time-dependent variables were substance use disorder, drugs for substance use disorder, mood disorder, use of antidepressants, cardiovascular disorder, drugs for cardiovascular disorders, diabetes, drugs for diabetes and cancer. The time-dependent variables changed at the time of their first occurrence and were time-lasting (permanent). To illustrate this point, after a diagnosis of a mood disorder or the dispension of a drug for cardiovascular problems, this variable remained 'yes' for the rest of the follow-up period. 


\section{Statistical analysis}

For the main analyses, we used an incidence cohort, because follow-up can be started at the moment of the first registration of a diagnosis of NAPD. The analyses for cumulative use were conducted in the incidence cohort only, because we did not have information on the use of antipsychotics before the start of follow-up in the prevalence cohort. Cox proportional hazards regression with time-dependent variables was used to estimate hazard ratios and 95\% confidence intervals (CIs) for the association between exposure to antipsychotics and mortality (all-cause and cause-specific). All subjects were followed up from their first diagnosis, their 15th birthday, or from 1 January 1995, whichever occurred last, until death or 1 July 2014. To allow for the possibility of at least 1 year of follow-up, the latest entry date was 30 June 2013. Due to violation of the proportional hazards assumption, the Cox analyses were stratified by age at start of follow-up, sex, Drug Unknown, and in the prevalence cohort also by (registered) duration of illness before the start of the follow-up. The proportional hazards assumption for the Cox regression models was tested and evaluated by graphical assessment of smoothed hazard estimates plots. Clozapine monotherapy was used as reference. The analyses were performed with Stata. A two-tailed p-value of $<0.05$ was considered statistically significant for all tests. Both the analyses of current use and the analyses of cumulative use were conducted for three types of mortality: (1) all-cause mortality; (2) mortality due to suicide; and (3) cardiovascular mortality.

We used two different types of adjustment in order to test the hypothesis that somatic comorbidity and the treatment thereof may influence the association between clozapine and mortality. In the first model, the results were adjusted for the time-fixed variables age at entry, sex, type of NAPD, and psychiatric hospitalization before start of follow-up, and for the time-dependent variables mood disorder, substance use disorder, malignant neoplasms, drugs for mood disorder, and drugs for substance use disorder. We adjusted for type of NAPD and psychiatric hospitalization, because they are proxies for illness severity. We adjusted for malignant neoplasms, in order to make sure that any difference between antipsychotics was not due to the occurrence of neoplasms. In the prevalence cohort, we also adjusted for time since first (registered) NAPD diagnosis. In the second model, the results were also adjusted for cardiovascular and diabetic comorbidity (diagnosis and dispension of drugs) as time-dependent variables.

\section{Compliance with ethical standards}

Statistics Denmark and the Danish Health Data Authority approved use of the data for the study. Ethics approval is not required for retrospective register-based studies in Denmark. 


\section{Results}

\section{Description of cohorts}

The incidence and prevalence cohorts consisted of 22110 patients and 50881 patients, respectively. Table 1 shows the baseline characteristics. In the 19.5-year observation period, the mean duration of follow-up in the incidence cohort was 8.8 years (range $0-19.5 ; 195461$ person-years) and in the prevalence cohort 11.3 years (range 0-19.5; 572617 person-years). During the follow-up period, 3612 individuals in the incidence cohort died, 479 due to suicide and 917 from a cardiovascular cause. For the analysis of current use, 375 deaths that occurred after 2 weeks of hospitalization were excluded, resulting in 3237 deaths ( 407 due to suicide and 851 from a cardiovascular cause). After the exclusion of 1439 individuals who died after 2 weeks of hospitalization, in total 11 948 patients died (1 050 deaths due to suicide and 3601 from a cardiovascular cause) in the prevalence cohort. Supplementary Table $S 1$ shows the number of person-years and all-cause and cause-specific deaths, per category of antipsychotic use, for current use in the incidence cohort. Supplementary Table S2 shows the numbers of cause-specific deaths for the categories of cumulative use in the incidence cohort. There was a large difference in the number of suicides associated with current use of clozapine (Table S1, $\mathrm{N}=5$ ) and cumulative use of clozapine up to 1 year (Table S2, N=113).

\section{Current use}

\section{All-cause mortality}

Figure 1 shows the results for the incidence and the prevalence cohorts, with two types of adjustment. In the incidence cohort, clozapine was not associated with a lower mortality, except when compared to hospital-delivered antipsychotics. In the prevalence cohort, although the HRs were closer to 1 than in the incidence cohort, the confidence intervals were smaller and the HRs for risperidone, polypharmacy including clozapine, and hospital-delivered antipsychotics were significantly higher than that for clozapine (monotherapy). The figure shows that the differences in results between the two types of adjustment were very small. The additional analysis with all monotherapies combined (the last bar in the figure) showed no significant effect of clozapine in either cohort. 
Table 1. Baseline characteristics of all people in Denmark first diagnosed with a non-affective psychotic disorder between 1 January 1995 and 1 July 2013 (incidence cohort) and of all people ever diagnosed with a non-affective psychotic disorder (prevalence cohort).

\begin{tabular}{|c|c|c|}
\hline & $\begin{array}{l}\text { Incidence cohort } \\
\mathrm{N}=22110\end{array}$ & $\begin{array}{l}\text { Prevalence cohort } \\
\mathrm{N}=50881\end{array}$ \\
\hline Male & $53.4 \%$ & $55.6 \%$ \\
\hline Age at inclusion in cohort (SD) & $35.7(16.4)$ & $39.7(16.5)$ \\
\hline Age of first diagnosis of psychosis (SD) & $35.7(16.4)$ & $34.1(14.7)$ \\
\hline \multicolumn{3}{|l|}{ Diagnosis at start follow-up } \\
\hline Schizophrenia (ICD-8 295) & N.A. & $15310(30.1 \%)$ \\
\hline Schizophrenia (ICD-10 F20) & $16611(75.1 \%)$ & $23627(46.4 \%)$ \\
\hline Schizoaffective disorders (ICD-10 F25) & $2076(9.4 \%)$ & $2926(5.8 \%)$ \\
\hline Unspecified psychosis (ICD-8 299) & N.A. & $3946(7.8 \%)$ \\
\hline $\begin{array}{l}\text { Other psychotic disorder not due to a sul } \\
\text { physiological condition (ICD-10 F28) }\end{array}$ & $926(4.2 \%)$ & $1393(2.7 \%)$ \\
\hline $\begin{array}{l}\text { Unspecified psychosis not due to a substa } \\
\text { physiological condition (ICD-10 F29) }\end{array}$ & $2497(11.3 \%)$ & $3679(7.2 \%)$ \\
\hline \multicolumn{3}{|l|}{ Diagnosis at end of follow-up } \\
\hline Schizophrenia (ICD-8 295) & N.A & $16384(32.2 \%)$ \\
\hline Schizophrenia (ICD-10 F20) & $17994(81.4 \%)$ & $26163(51.4 \%)$ \\
\hline Schizoaffective disorders (ICD-10 F25) & $1660(7.5 \%)$ & $2395(4.7 \%)$ \\
\hline Unspecified psychosis (ICD-8 299) & N.A. & $2360(4.6 \%)$ \\
\hline $\begin{array}{l}\text { Other psychotic disorder not due to a sul } \\
\text { physiological condition (ICD-10 F28) }\end{array}$ & $702(3.2 \%)$ & $1067(2.1 \%)$ \\
\hline $\begin{array}{l}\text { Unspecified psychosis not due to a substa } \\
\text { physiological condition (ICD-10 F29) }\end{array}$ & $1754(7.9 \%)$ & $2513(4.9 \%)$ \\
\hline Mood disorder & $5360(24.2 \%)$ & $10855(21.3 \%)$ \\
\hline Psychiatric Hospitalization & $16365(74.0 \%)$ & $42357(83.3 \%)$ \\
\hline Substance use disorder & $6014(27.2 \%)$ & $12352(24.3 \%)$ \\
\hline Drugs for substance use disorder & $554(2.5 \%)$ & $668(1.3 \%)$ \\
\hline Antidepressant drug & $6102(27.6 \%)$ & $7702(15.1 \%)$ \\
\hline Diabetes mellitus & $549(2.5 \%)$ & $1185(2.3 \%)$ \\
\hline Circulatory system diseases & $2062(9.3 \%)$ & $3992(7.9 \%)$ \\
\hline Malignant Neoplasms & $438(2.0 \%)$ & $1055(2.1 \%)$ \\
\hline
\end{tabular}




\section{Suicide}

Supplementary Table S1 shows that none of the clozapine users in the incidence cohort died by suicide in 6 years after the first diagnosis (during $706+1017=1723$ person-years). The results of the analyses for current antipsychotic use are shown in Figure 2. In the incidence cohort, mortality due to suicide during the use of clozapine was lower compared to that during the use of other antipsychotics, but this difference was only significant for the comparison with users of hospital-delivered antipsychotics. In the prevalence cohort, all categories of current antipsychotic use were associated with a significantly higher mortality compared to clozapine. These differences were larger than in the incidence cohort and the confidence intervals were smaller. Accordingly, mortality for all monotherapies combined was significantly higher compared to clozapine in the prevalence cohort $\left(\mathrm{HR}_{\mathrm{adj}}=2.20\right.$; 95\% CI 1.35-3.59), but not in the incidence cohort $\left(\mathrm{HR}_{\mathrm{adj}}=1.76\right.$; 95\% CI 0.72-4.32). Adjustment for somatic comorbidity did not affect the results.

Figure 1. Adjusted hazard ratios for all-cause mortality during current use of antipsychotics compared to current use of clozapine (reference), between January 1995 and July 2014, in Danish incidence and prevalence cohorts of patients with a non-affective psychotic disorder.

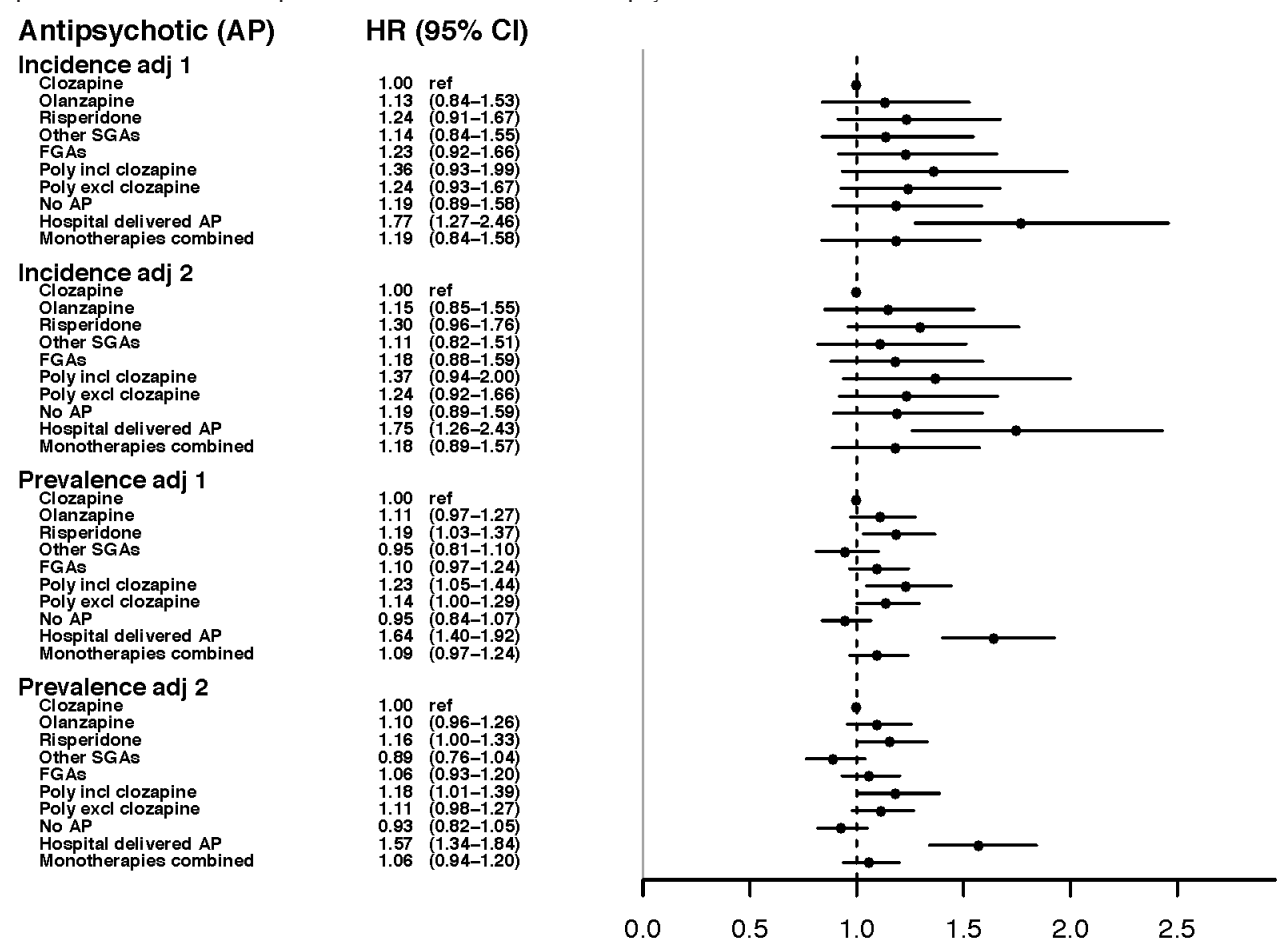

Adj 1: Adjusted for age, sex, type of non-affective psychotic disorder, mood disorder, substance use disorder, psychiatric hospitalization, and in the prevalence cohort for duration of illness.

Adj 2: Also adjusted for somatic comorbidity and the treatment of somatic disorders. 
Figure 2. Adjusted hazard ratios for mortality due to suicide during current use of antipsychotics compared to current use of clozapine (reference), between January 1995 and July 2014, in Danish incidence and prevalence cohorts of patients with a non-affective psychotic disorder. The observation period was from January 1995 to July 2014.

Antipsychotic (AP)
Incidence adj 1
Clozapine
Olanzapine
Risperidone
Other SGAs
FGAs
Poly incl dozapine
Poly excl clozapine
No AP
Hospital delivered AP
Monotherapies combined
Incidence adj 2
Clozapine
Olanzapine
Risperidone
Other SGAs
FGAs
Poly incl clozapine
Poly excl clozapine
No AP
Hospital delivered AP
Monotherapies combined
Prevalence adj 1
Clozapine
Olanzapine
Risperidone
Other SGAs
FGAs
Poly incl clozapine
Poly excl clozapine
No AP
Hospital delivered AP
Monotherapies combined
Prevalence adj 2
Clozapine
Olanzapine
Risperidone
Other SGAs
FGAs
Poly incl dozapine
Poly excl clozapine
No AP
Hospital delivered AP
Monotherapies combined

$\mathrm{HR}(95 \% \mathrm{Cl})$

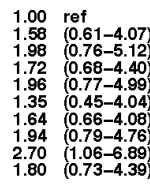

1.00 ref

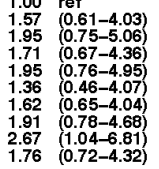

1.00 ref

$2.17(1.28-3.68)$

$2.01(1.16-3.48)$

$2.27 \quad(1.37-3.74)$

$2.00(1.21-3.29)$

$2.11(1.29-3.44)$

$2.22(1.36-3.62)$

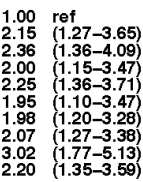

Adj 1: Adjusted for age, sex, type of non-affective psychotic disorder, mood disorder, substance use disorder, psychiatric hospitalization, and in the prevalence cohort for duration of illness.

Adj 2: Also adjusted for somatic comorbidity and the treatment of somatic disorders.

\section{Cardiovascular mortality}

In the incidence cohort, the hazard of cardiovascular mortality was lower with clozapine than with most other antipsychotics, but the confidence intervals were broad and included 1 (Figure 3). As for the prevalence cohort, cardiovascular mortality associated with clozapine was significantly lower in the first adjusted model than that associated with risperidone and hospital-delivered antipsychotics. However, after adjustment for cardiovascular and diabetic comorbidity, the HRs slightly decreased towards 1 and were no longer statistically significant. 
Figure 3. Adjusted hazard ratios for cardiovascular mortality during current use of antipsychotics compared to current use of clozapine (reference), between January 1995 and July 2014, in Danish incidence and prevalence cohorts of patients with a non-affective psychotic disorder. The observation period was from January 1995 to July 2014.

\begin{tabular}{|c|c|c|}
\hline Antipsychotic (AP) & \multicolumn{2}{|c|}{$\mathrm{HR}(95 \% \mathrm{Cl})$} \\
\hline $\begin{array}{l}\text { Incldence adj } 1 \\
\text { Clozapine } \\
\text { Olanzapine } \\
\text { Risperidone } \\
\text { Other SGAs } \\
\text { FGAs } \\
\text { Poly incl clozapine } \\
\text { Poly excl clozapine } \\
\text { No AP } \\
\text { Hospital delivered AP } \\
\text { Monotherapies combined }\end{array}$ & $\begin{array}{l}1.00 \\
1.32 \\
1.50 \\
1.35 \\
1.47 \\
1.83 \\
1.56 \\
1.46 \\
0.91 \\
1.41\end{array}$ & $\begin{array}{l}\text { ref } \\
(0.69-2.52) \\
(0.78-2.88) \\
(0.69-2.63) \\
(0.77-2.81) \\
(0.80-4.19) \\
(0.82-2.99) \\
(0.77-2.75) \\
(0.38-2.21) \\
(0.75-2.65)\end{array}$ \\
\hline $\begin{array}{l}\text { Incidence adj } 2 \\
\text { Clozapine } \\
\text { Olanzapine } \\
\text { Risperidone } \\
\text { Other SGAs } \\
\text { FGAs } \\
\text { Poly incl clozapine } \\
\text { Poly excl clozapine } \\
\text { No AP } \\
\text { Hospital delivered AP } \\
\text { Monotherapies combined }\end{array}$ & $\begin{array}{l}1.00 \\
1.14 \\
1.44 \\
1.11 \\
1.29 \\
1.62 \\
1.37 \\
1.38 \\
0.78 \\
1.25\end{array}$ & $\begin{array}{l}\text { ref } \\
(0.59-2.19) \\
(0.75-2.78) \\
(0.57-2.18) \\
(0.67-2.45) \\
(0.71-3.72) \\
(0.72-2.61) \\
(0.73-2.60) \\
(0.32-1.88) \\
(0.66-2.34)\end{array}$ \\
\hline $\begin{array}{l}\text { Prevalence adj } 1 \\
\text { Clozapine } \\
\text { Olanzapine } \\
\text { Risperidone } \\
\text { Other SGAs } \\
\text { FGAs } \\
\text { Poly incl clozapine } \\
\text { Poly excl cozapine } \\
\text { No AP } \\
\text { Hospital delivered AP } \\
\text { Monotherapies combined }\end{array}$ & $\begin{array}{l}1.00 \\
1.21 \\
1.41 \\
1.02 \\
1.24 \\
1.33 \\
1.28 \\
1.05 \\
1.49 \\
1.24\end{array}$ & $\begin{array}{l}\text { ref } \\
(0.92-1.59) \\
(1.07-1.87) \\
(0.75-1.40) \\
(0.96-1.60) \\
(0.96-1.85) \\
(0.99-1.66) \\
(0.82-1.36) \\
(1.04-2.13) \\
(0.96-1.59)\end{array}$ \\
\hline $\begin{array}{l}\text { Prevalence adj } 2 \\
\text { Clozapine } \\
\text { Olanzapine } \\
\text { Risperidone } \\
\text { Other SGAs } \\
\text { FGAs } \\
\text { Poly incl clozapine } \\
\text { Poly excl clozapine } \\
\text { No AP } \\
\text { Hospital delivered AP } \\
\text { Monotherapies combined }\end{array}$ & $\begin{array}{l}1.00 \\
1.05 \\
1.22 \\
0.82 \\
1.07 \\
1.19 \\
1.13 \\
0.95 \\
1.33 \\
1.06\end{array}$ & $\begin{array}{l}\text { ref } \\
(0.80-1.39) \\
(0.92-1.61) \\
(0.60-1.12) \\
(0.83-1.38) \\
(0.86-1.65) \\
(0.87-1.47) \\
(0.74-1.22) \\
(0.93-1.90) \\
(0.83-1.37)\end{array}$ \\
\hline
\end{tabular}

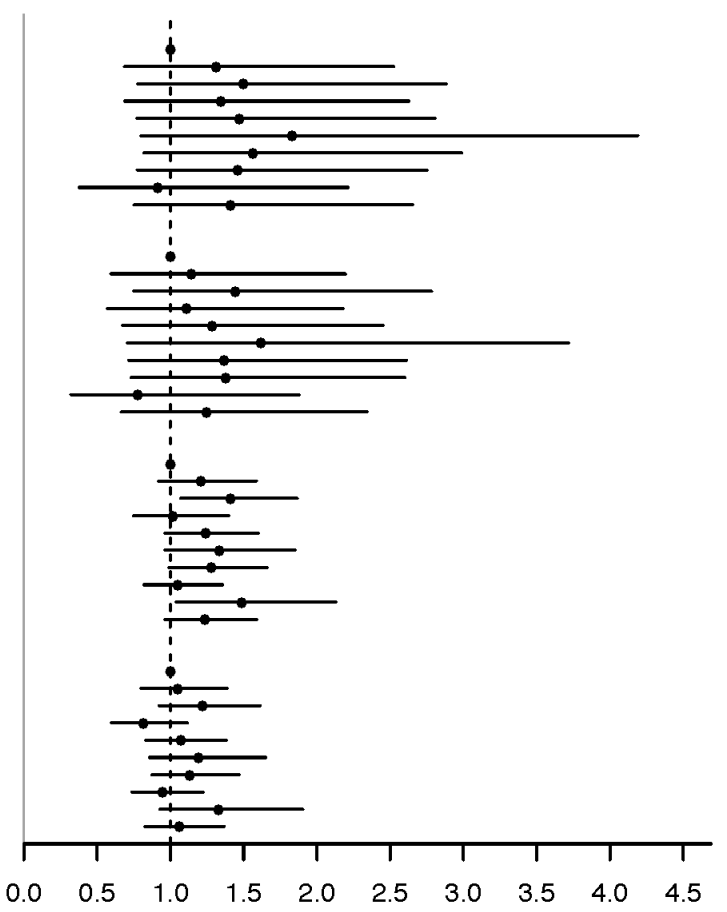

Adj 1: Adjusted for age, sex, type of non-affective psychotic disorder, mood disorder, substance use disorder, psychiatric hospitalization, and in the prevalence cohort for duration of illness.

Adj 2: Also adjusted for somatic comorbidity and the treatment of somatic disorders.

\section{Cumulative use}

\section{All-cause mortality}

Figure 4 shows that cumulative use of clozapine for up to 1 year was associated with a higher all-cause mortality than cumulative use of most other antipsychotics. This difference cannot be attributed to myocarditis, because there were no cases during this 1 -year period. This difference in all-cause mortality was not present after longer use of the drugs. Adjustment for somatic comorbidity had a minimal effect (figure with first type of adjustment not shown). 
Figure 4. Adjusted hazard ratios for all-cause mortality after cumulative use of antipsychotics compared to cumulative use of clozapine (reference), between January 1995 and July 2014, in a Danish incidence cohort of patients with a non-affective psychotic disorder. Comparisons with clozapine were done within each of the groups distinguished by the same length of antipsychotic use: 0-1, 1-3, 3-6, 6-10 years, and more than 10 years. The observation period was from January 1995 to July 2014.

\section{Antipsychotic (AP)HR (95\% Cl)}
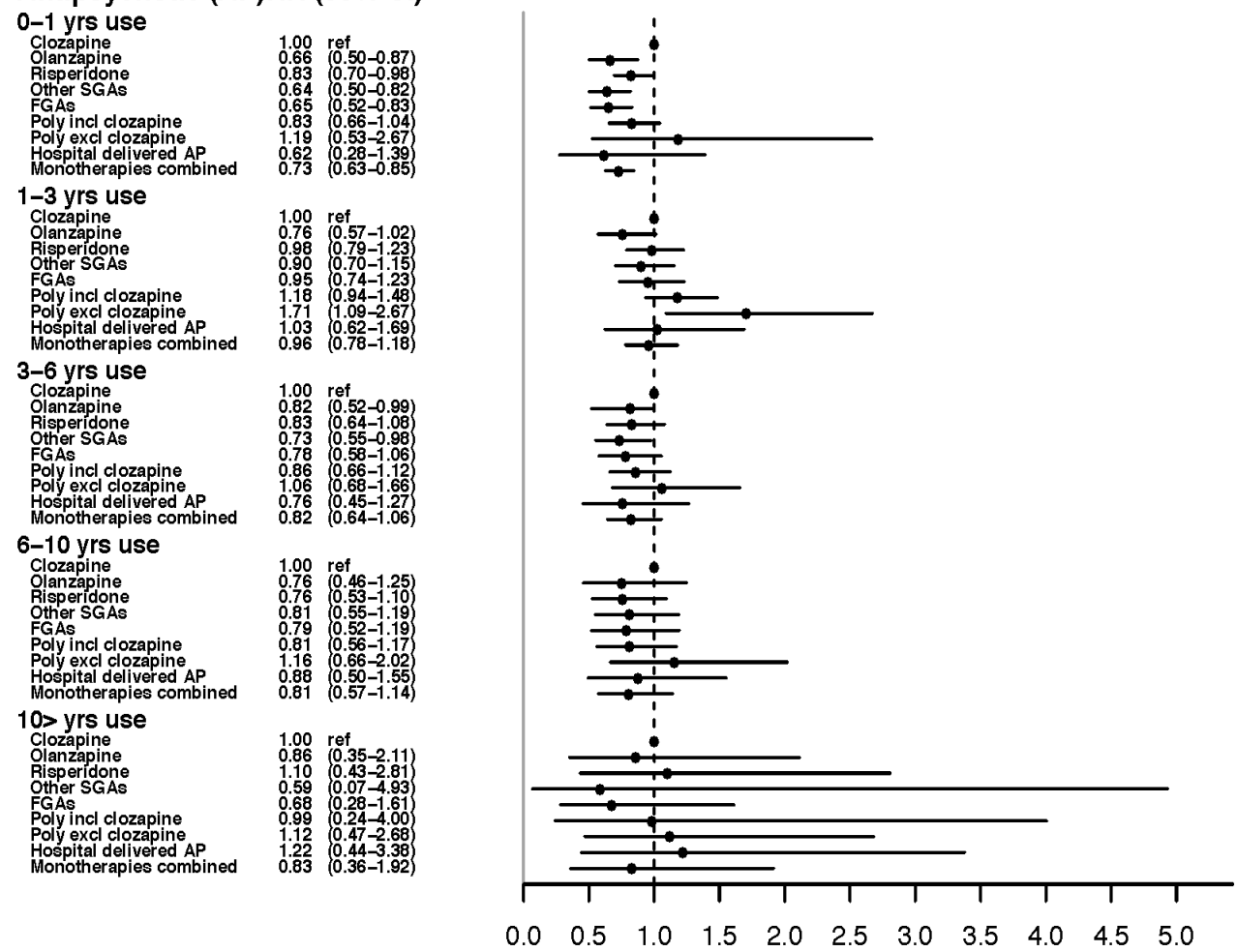

Adjusted for age, sex, type of non-affective psychotic disorder, mood disorder, substance use disorder, psychiatric hospitalization, somatic comorbidity, the treatment of somatic disorders.

\section{Suicide}

The fully adjusted analysis of cumulative use also shows a significantly higher suicide rate among patients who had used clozapine for up to 1 year than among patients who had used polypharmacy including clozapine, olanzapine, other SGAs and all monotherapies combined for a similar time (see Figure S1). After cumulative use of more than 1 year, this difference in mortality due to suicide was no longer significant. Of the individuals who committed suicide, $24 \%$ had used clozapine monotherapy for longer than any other antipsychotic during a period up to 1 year (113 of 479 suicides) (Table S2). 


\section{Cardiovascular deaths}

Except for cumulative use of polypharmacy excluding clozapine (1 to 3 years), there were no significant differences between use of clozapine and use of other antipsychotics in cardiovascular mortality, irrespective of the duration of use or adjustment for somatic comorbidity (see Figure S2).

\section{Discussion}

Our first objective was to investigate whether mortality (all-cause and cause-specific) associated with "current" use of clozapine was lower than that associated with "current" use of other antipsychotics. A second objective was to assess whether findings were similar when "cumulative" use was analyzed instead of "current" use. In general, current use of clozapine was not associated with a lower all-cause or cardiovascular mortality in the incidence or prevalence cohort. In both cohorts, however, the numbers of suicides were lower with current use of clozapine. Remarkably, cumulative use of clozapine for 0-1 year was associated with a higher mortality (all-cause and suicide) compared to cumulative use of almost all other categories. Cumulative use of clozapine for 6 years or longer was not associated with a higher all-cause or cardiovascular mortality, despite its metabolic side-effects. Adjustment for the presence and treatment of somatic comorbidity hardly affected the results.

\section{Interpretation and comparison with other studies \\ Current use}

Our finding of no significantly lower all-cause mortality among users of clozapine is consistent with several other large database studies (Ringback Weitoft et al., 2014; Stroup, Gerhard, Crystal, Huang, \& Olfson, 2016; Taipale et al., 2017; Taipale et al., 2020), but not with the study that most closely resembled our study in design, the FIN11 study (Tiihonen et al., 2009). It is possible that results in different countries vary due to differences in prescription rates (more or less restrictive use) or differences in the National registers that were used. However, the FIN20 study (Taipale et al., 2020) yielded different results than the FIN11 study (Tiihonen et al., 2009), despite similar inclusion criteria and year of start of follow-up. See Table S3 for differences and similarities between the Finnish studies and the present study. The FIN11 study reported a large reduction of mortality among users of this drug, for all-cause and cause specific mortality. As already pointed out by de Hert et al. (2010), the authors disregarded two-thirds of all deaths, by excluding deaths after a hospitalization of more than 2 days. In the FIN20 study, follow-up time was censored after 7 days of hospital admission instead of 2 days. The results of the FIN20 study show that clozapine was no longer associated with a significantly lower mortality compared to all other categories of antipsychotics. If these different findings of the Finnish 
studies are partly caused by the difference in censoring deaths during hospital admission, this may also explain the differences in results between our study and the FIN11 study, given that we excluded deaths after 14 days of hospitalization. In two studies that were restricted to treatment-resistant patients (Cho et al., 2019; Wimberley et al., 2017), a significantly lower mortality associated with clozapine use was found. However, mortality was only significantly lower when comparing the use of clozapine to non-use of clozapine (including no use of any antipsychotic).

Our finding of a lower suicide rate during the use of clozapine is in line with the results of many previous studies (Hayes et al., 2015; Hennen \& Baldessarini, 2005; Taipale et al., 2020; Tiihonen et al., 2009; Walker et al., 1997). The differences in hazard ratios for current use between the incidence and the prevalence cohorts in our study were slightly smaller than expected, given the possible survivor bias in the prevalence cohort. The HRadj for mortality due to suicide during use of all monotherapies combined dropped from 2.20 (CI 1.35-3.59) in the prevalence cohort to 1.76 (CI 0.72-4.32) in the incidence cohort, but the confidence intervals were broad and overlapping. In a sensitivity analysis of the FIN20 study, excluding the first ten years of follow-up, clozapine dropped from third to seventh place in the ranking of the most beneficial antipsychotics and all-cause mortality. Thus, survivorship bias may have influenced the results of the FIN20 study.

\section{Cumulative use}

There are two studies to which we can compare our results on cumulative use, despite different designs and statistical analyses. The first is the FIN11 study (Tiihonen et al., 2009), which also compared cumulative use of different categories of antipsychotics. In this study a prevalence cohort was used, while we used an incidence cohort with complete information on drug use before start follow-up. In addition, the analysis strategies differed. In the FIN11 study, all episodes of use were included in the analysis and relative risks were calculated. In such an analysis, some people can be in two different categories at a certain time point and may have been compared with themselves. In our Cox regression analyses, patients were put in exclusive categories at each time point and, thus, were never compared to themselves at a single time point. Consequently, they were in the category of the drug they had used for the longest time and the use of other antipsychotics was disregarded. The second study, the FIN20 study (Taipale et al., 2020), compared cumulative use of clozapine only to cumulative use of any antipsychotic and calculated odds ratios. Neither the FIN11 nor the FIN20 reported significant differences in cardiovascular mortality between cumulative use of clozapine and cumulative use of other antipsychotics. This is in line with the results on cumulative use of our study. 
Remarkably, cumulative use of clozapine for more than 6 years did not seem to increase cardiovascular mortality, despite its metabolic side-effects. This is in line with the findings of Kelly et al. (2010), who did not find significant differences in cardiovascular mortality between those who started clozapine and those who started risperidone after a 6- to 10-year observation period. This may be explained by the effectiveness of clozapine against psychotic symptoms and the subsequent reduction of stress and substance abuse.

\section{Current and cumulative use combined}

Our findings of higher overall mortality and higher suicide mortality among patients who had used clozapine for up to 1 year, in combination with the lower mortality during current use of this drug, suggest that the extra deaths occurred after the termination of clozapine use. Concerning suicide, Tables $S 1$ and S2 show that only 5 suicides occurred during clozapine use, while 113 suicides occurred after 0-1 year of cumulative clozapine use. This finding is in line with the previous report from Denmark (Wimberley et al., 2017) of an increased risk of death after termination versus during clozapine use (HR 2.65, 95\% CI 1.47-4.78). The authors of this report deemed it likely that the excess mortality rate in the first year or even within 3 months of discontinuation was due to causes other than suicide, because clozapine could have been discontinued due to severe medical conditions related or unrelated to clozapine treatment. However, we do find an increased risk of suicide after clozapine discontinuation, a phenomenon previously described by Patchan, Richardson, Vyas, \& Kelly (2015) and Walker et al. (1997). Walker et al. (1997) reported a markedly decreased suicide rate during current use compared to past use of clozapine (standardized mortality ratio 0.17 ; CI $0.10-0.30$ ). Their conclusion that clozapine decreases the suicide rate among users is only half the story, because we found that the suicide rate associated with cumulative clozapine use was higher than that for cumulative use of other antipsychotics. As clozapine is a drug of last resort, stopping it may lead to relapse or give rise to despair and an increased suicidality. Furthermore, since clozapine is indicated for suicidal patients, such patients are more likely to start using clozapine (i.e., confounding by indication). This could contribute to the high risk of suicide after termination of this drug. There is no good evidence that the increased risk of suicide after termination is due to a rebound effect or to acute clozapine withdrawal.

\section{Strengths and limitations}

To our knowledge, this is the first large study to compare mortality associated with cumulative use of different antipsychotics using a representative incidence cohort with complete information on medication use from the start of treatment. In addition, the incidence cohort of 22110 patients is larger than in any other study on clozapine and mortality. With a maximum of 19.5 years, the follow-up time of the cohort was very long. We included $90 \%$ of all deaths in the analyses of current use and this is the first 
study to adjust the results for somatic comorbidity and somatic treatment. To ensure that differences in findings between our study and other studies are not due to the use of an incidence cohort, we repeated our analysis in a prevalence cohort.

Several limitations need to be mentioned. First, the databases we used did not include direct information on disease severity or lifestyle factors. Second, there was no information on medication use during hospitalization, as a result of which we had to exclude deaths during longer hospitalizations. Third, the prescription database consists of dispensed drugs, it is unknown whether or not the patients have actually used the drugs. Fourth, psychiatric registrations before 1969 were incomplete, leading to missing information about age at disease onset for many patients in the prevalence cohort and thus to residual confounding. Fifth, despite the long observation period (19.5 years), the power of the analyses of cause-specific mortality in the incidence cohort was not optimal. Sixth, the category 'non-users of antipsychotics' is likely to consist of patients with mild symptoms not needing antipsychotics and patients with more severe symptoms who refuse treatment. We were not able to distinguish between these groups. Seventh, we did not correct for multiple testing. Since almost none of our results were significant, a correction for multiple testing would not have changed this result. Eighth, in the incidence cohort analyses, we excluded migrants because information on diagnoses and antipsychotic use before migrating was unknown. Ninth, because the follow-up started at the moment of first diagnosis of NAPD, previous psychotropic and antipsychotic drug use was disregarded. Tenth, our definition of cumulative use does not take into account whether this drug has been discontinued and the length of time since discontinuation. Finally, there may have been residual confounding. Since clozapine is more commonly prescribed to severely ill patients, we adjusted for type of diagnosis and history of hospitalization. We do not know whether this adjustment was adequate, because it hardly changed the results. More adequate adjustments for severity of illness could therefore have led to more favorable outcomes for clozapine.

To conclude, we found no major differences in mortality between users of clozapine and users of other antipsychotics. This should encourage clinicians to prescribe clozapine to the patients who need it. Moreover, our findings add to an increasing body of evidence that clozapine use protects against suicide. This protective effect is lost when clozapine is discontinued. Clinicians should carefully monitor patients from whom clozapine has been withdrawn or consider a clozapine re-challenge. 


\section{References}

Cho, J., Hayes, R. D., Jewell, A., Kadra, G., Shetty, H., MacCabe, J. H., \& Downs, J. (2019). Clozapine and all-cause mortality in treatment-resistant schizophrenia: a historical cohort study. Acta Psychiatr Scand, 139(3), 237-247. doi:10.1111/acps.12989

De Berardis, D., Rapini, G., Olivieri, L., Di Nicola, D., Tomasetti, C., Valchera, A., . . Di Giannantonio, M. (2018). Safety of antipsychotics for the treatment of schizophrenia: a focus on the adverse effects of clozapine. Ther Adv Drug Saf, 9(5), 237-256. doi:10.1177/2042098618756261

De Hert, M., Correll, C. U., \& Cohen, D. (2010). Do antipsychotic medications reduce or increase mortality in schizophrenia? A critical appraisal of the FIN-11 study. Schizophr Res, 117(1), 68-74. doi:10.1016/j.schres.2009.12.029

Hayes, R. D., Downs, J., Chang, C. K., Jackson, R. G., Shetty, H., Broadbent, M., . . Stewart, R. (2015). The effect of clozapine on premature mortality: an assessment of clinical monitoring and other potential confounders. Schizophr Bull, 41(3), 644-655. doi:10.1093/schbul/sbu120

Hennen, J., \& Baldessarini, R. J. (2005). Suicidal risk during treatment with clozapine: a meta-analysis. Schizophr Res, 73(2-3), 139-145. doi:10.1016/j.schres.2004.05.015

Hirsch, L., Yang, J., Bresee, L., Jette, N., Patten, S., \& Pringsheim, T. (2017). Second-Generation Antipsychotics and Metabolic Side Effects: A Systematic Review of Population-Based Studies. Drug Saf, 40(9), 771-781. doi:10.1007/s40264-017-0543-0

Howes, O. D., Vergunst, F., Gee, S., McGuire, P., Kapur, S., \& Taylor, D. (2012). Adherence to treatment guidelines in clinical practice: study of antipsychotic treatment prior to clozapine initiation. $\mathrm{Br} J$ Psychiatry, 201(6), 481-485. doi:10.1192/bjp.bp.111.105833

Kelly, D. L., McMahon, R. P., Liu, F., Love, R. C., Wehring, H. J., Shim, J. C., . . Conley, R. R. (2010). Cardiovascular disease mortality in patients with chronic schizophrenia treated with clozapine: a retrospective cohort study. J Clin Psychiatry, 71(3), 304-311. doi:10.4088/JCP.08m04718yel

Kiviniemi, M., J. Suvisaari, H. Koivumaa-Honkanen, U. Häkkinen, M. Isohanni, H. Hakkoe. (2013). Antipsychotics and mortality in first-onset schizophrenia: Prospective Finnish register study with 5-year follow-up. Schizophr Res, 150, 274-280.

Patchan, K. M., Richardson, C., Vyas, G., \& Kelly, D. L. (2015). The risk of suicide after clozapine discontinuation: Cause for concern. Ann Clin Psychiatry, 27(4), 253-256.

Ringback Weitoft, G., Berglund, M., Lindstrom, E. A., Nilsson, M., Salmi, P., \& Rosen, M. (2014). Mortality, attempted suicide, re-hospitalisation and prescription refill for clozapine and other antipsychotics in Sweden-a register-based study. Pharmacoepidemiol Drug Saf, 23(3), 290-298. doi: $10.1002 /$ pds. 3567

Stroup, T. S., Gerhard, T., Crystal, S., Huang, C., \& Olfson, M. (2016). Comparative Effectiveness of Clozapine and Standard Antipsychotic Treatment in Adults With Schizophrenia. Am J Psychiatry, 173(2), 166-173. doi:10.1176/appi.ajp.2015.15030332

Taipale, H., Mittendorfer-Rutz, E., Alexanderson, K., Majak, M., Mehtala, J., Hoti, F., . . Tiihonen, J. (2017). Antipsychotics and mortality in a nationwide cohort of 29,823 patients with schizophrenia. Schizophr Res. doi:10.1016/j.schres.2017.12.010 
Taipale, H., Tanskanen, A., Mehtala, J., Vattulainen, P., Correll, C. U., \& Tiihonen, J. (2020). 20-year follow-up study of physical morbidity and mortality in relationship to antipsychotic treatment in a nationwide cohort of 62,250 patients with schizophrenia (FIN20). World Psychiatry, 19(1), 61-68. doi:10.1002/wps.20699

Termorshuizen, F., Wierdsma, A. I., Smeets, H. M., Visser, E., Drukker, M., Nijman, H., \& Sytema, S. (2013). Cause-specific mortality among patients with psychosis: disentangling the effects of age and illness duration. Psychosomatics, 54(6), 536-545. doi:10.1016/j.psym.2013.05.011

Tiihonen, J., Lonnqvist, J., Wahlbeck, K., Klaukka, T., Niskanen, L., Tanskanen, A., \& Haukka, J. (2009). 11-year follow-up of mortality in patients with schizophrenia: a population-based cohort study (FIN11 study). Lancet, 374(9690), 620-627. doi:10.1016/s0140-6736(09)60742-x

van der Zalm, Y. C., Termorshuizen, F., \& Selten, J. P. (2019). Concerns about bias in studies on clozapine and mortality. Schizophr Res, 204, 425-426. doi:10.1016/j.schres.2018.08.017

Vermeulen, J. M., van Rooijen, G., van de Kerkhof, M. P. J., Sutterland, A. L., Correll, C. U., \& de Haan, L. (2019). Clozapine and Long-Term Mortality Risk in Patients With Schizophrenia: A Systematic Review and Meta-analysis of Studies Lasting 1.1-12.5 Years. Schizophr Bull, 45(2), 315329. doi:10.1093/schbul/sby052

Walker, A. M., Lanza, L. L., Arellano, F., \& Rothman, K. J. (1997). Mortality in current and former users of clozapine. Epidemiology, 8(6), 671-677.

Wimberley, T., MacCabe, J. H., Laursen, T. M., Sorensen, H. J., Astrup, A., Horsdal, H. T., . . Stovring, H. (2017). Mortality and Self-Harm in Association With Clozapine in Treatment-Resistant Schizophrenia. Am J Psychiatry, 174(10), 990-998. doi:10.1176/appi.ajp.2017.16091097 


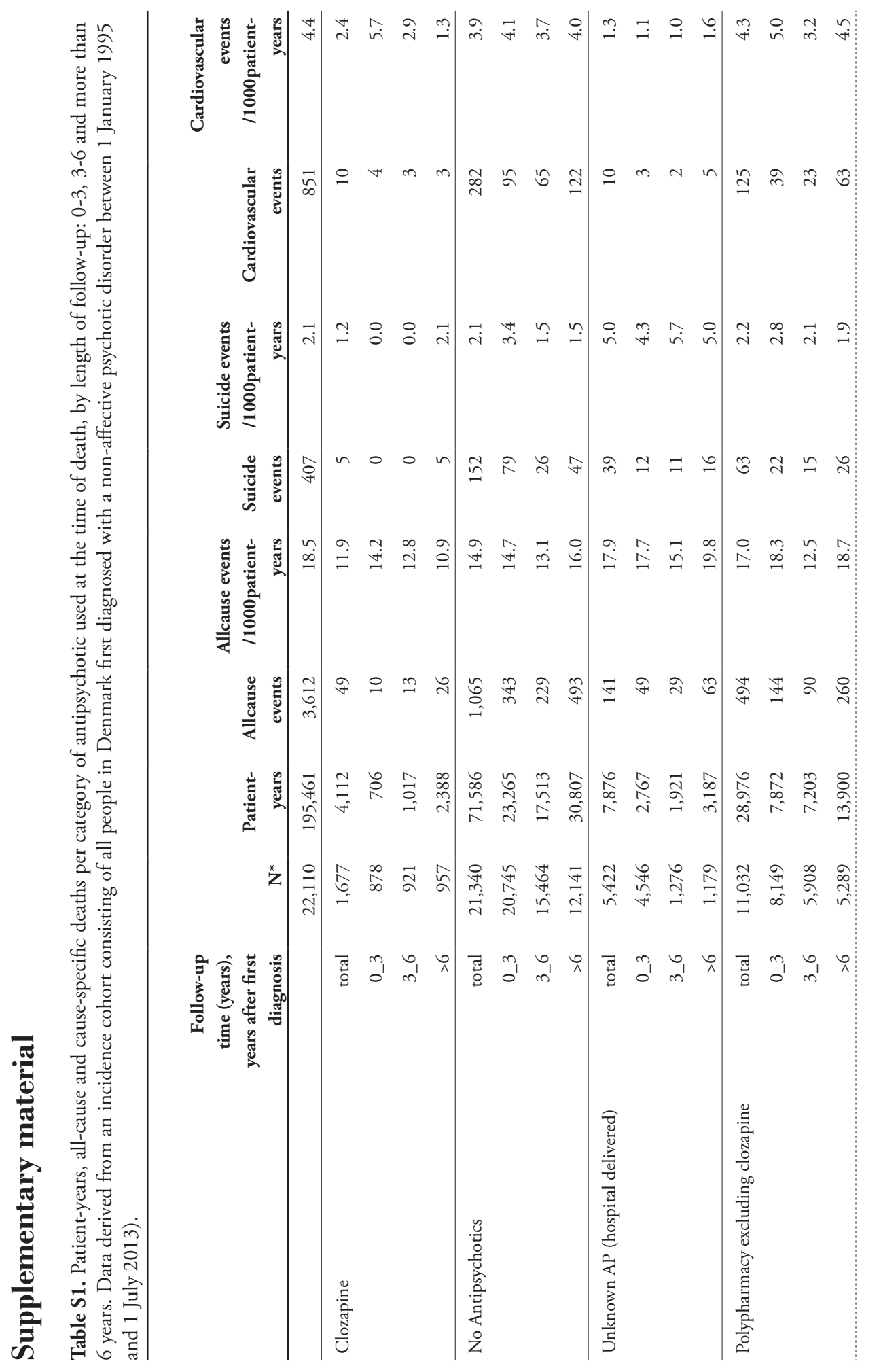




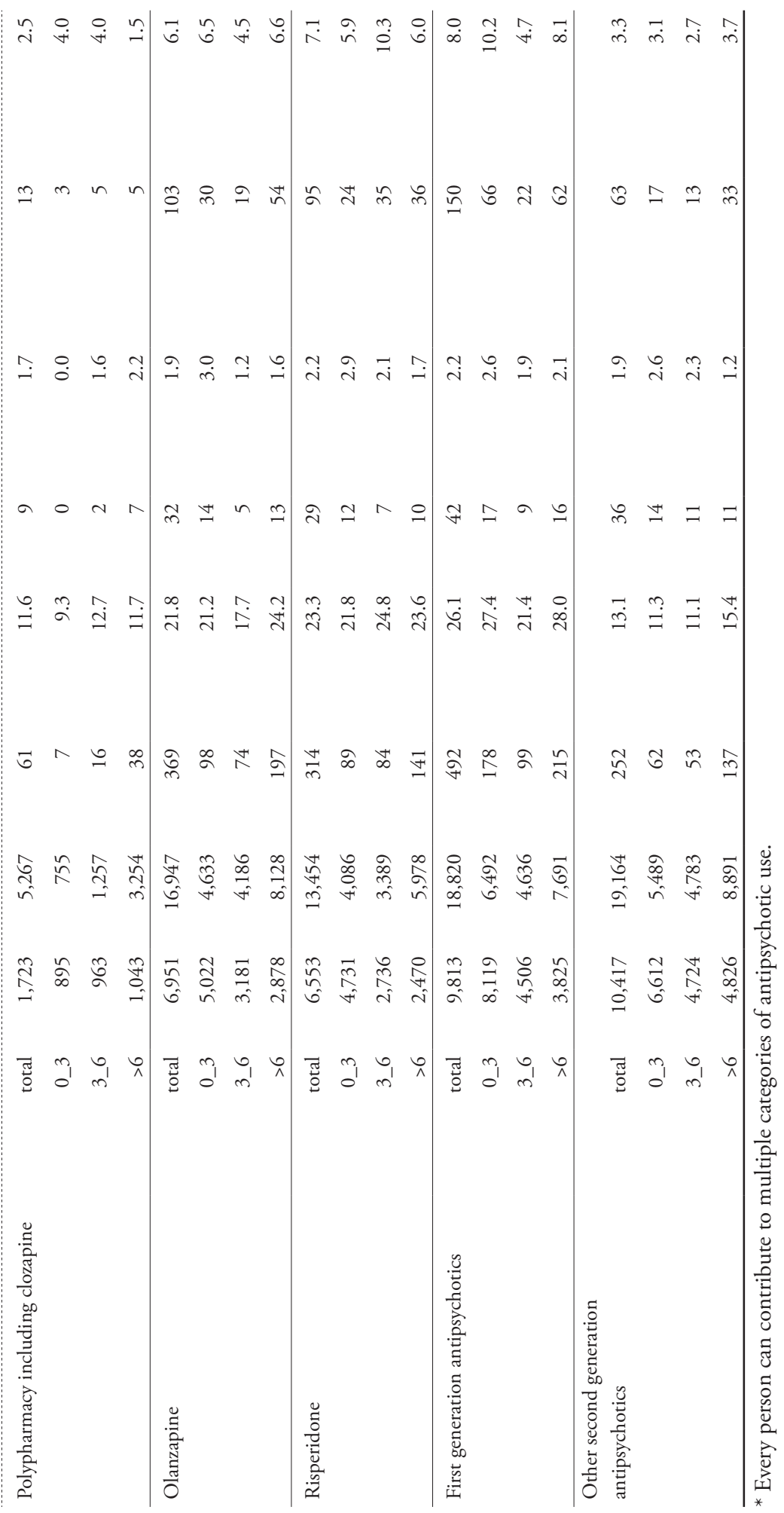


Table S2. Suicides and cardiovascular deaths in each category of cumulative use of antipsychotics in incidence cohort (all people in Denmark first diagnosed with a non-affective psychotic disorder between 1 January 1995 and 1 July 2013).

\begin{tabular}{|c|c|c|c|}
\hline Use during & Type of antipsychotic therapy & Suicides & $\begin{array}{l}\text { Cardiovascular } \\
\text { deaths }\end{array}$ \\
\hline & never used an antipsychotic drug & 14 & 38 \\
\hline $0-1$ year & clozapine monotherapy & 113 & 74 \\
\hline $0-1$ year & clozapine polytherapy & 18 & 23 \\
\hline $0-1$ year & olanzapine monotherapy & 13 & 19 \\
\hline $0-1$ year & risperidon monotherapy & 28 & 69 \\
\hline $0-1$ year & First generation antipsychotics monotherapy & 15 & 24 \\
\hline $0-1$ year & other second generation antipsychotics monotherapy & 9 & 23 \\
\hline $0-1$ year & polytherapy excluding clozapine & 1 & 1 \\
\hline $0-1$ year & unknown antipsychotic & 0 & 5 \\
\hline $1-3$ years & clozapine monotherapy & 34 & 19 \\
\hline $1-3$ years & clozapine polytherapy & 19 & 44 \\
\hline $1-3$ years & olanzapine monotherapy & 9 & 16 \\
\hline $1-3$ years & risperidon monotherapy & 22 & 80 \\
\hline $1-3$ years & First generation antipsychotics monotherapy & 19 & 38 \\
\hline $1-3$ years & other second generation antipsychotics monotherapy & 18 & 38 \\
\hline $1-3$ years & polytherapy excluding clozapine & 1 & 7 \\
\hline $1-3$ years & unknown antipsychotic & 0 & 4 \\
\hline $3-6$ years & clozapine monotherapy & 23 & 13 \\
\hline 3-6 years & clozapine polytherapy & 29 & 32 \\
\hline $3-6$ years & olanzapine monotherapy & 7 & 17 \\
\hline $3-6$ years & risperidon monotherapy & 18 & 64 \\
\hline 3-6 years & First generation antipsychotics monotherapy & 6 & 33 \\
\hline $3-6$ years & other second generation antipsychotics monotherapy & 11 & 44 \\
\hline $3-6$ years & polytherapy excluding clozapine & 4 & 6 \\
\hline 3-6 years & unknown antipsychotic & 4 & 1 \\
\hline $6-10$ years & clozapine monotherapy & 7 & 8 \\
\hline $6-10$ years & clozapine polytherapy & 12 & 31 \\
\hline $6-10$ years & olanzapine monotherapy & 1 & 8 \\
\hline $6-10$ years & risperidon monotherapy & 4 & 58 \\
\hline $6-10$ years & First generation antipsychotics monotherapy & 2 & 14 \\
\hline $6-10$ years & other second generation antipsychotics monotherapy & 5 & 20 \\
\hline $6-10$ years & polytherapy excluding clozapine & 4 & 2 \\
\hline $6-10$ years & unknown antipsychotic & 1 & 1 \\
\hline
\end{tabular}




\begin{tabular}{llll}
$>10$ years & clozapine monotherapy & 0 & 0 \\
$>10$ years & clozapine polytherapy & 0 & 2 \\
$>10$ years & olanzapine monotherapy & 2 & 6 \\
$>10$ years & risperidon monotherapy & 0 & 6 \\
$>10$ years & First generation antipsychotics monotherapy & 0 & 15 \\
$>10$ years & other second generation antipsychotics monotherapy & 0 & 0 \\
$>10$ years & polytherapy excluding clozapine & 1 & 14 \\
$>10$ years & unknown antipsychotic & 5 & 0 \\
\hline Total & & 479 & 917 \\
\hline
\end{tabular}




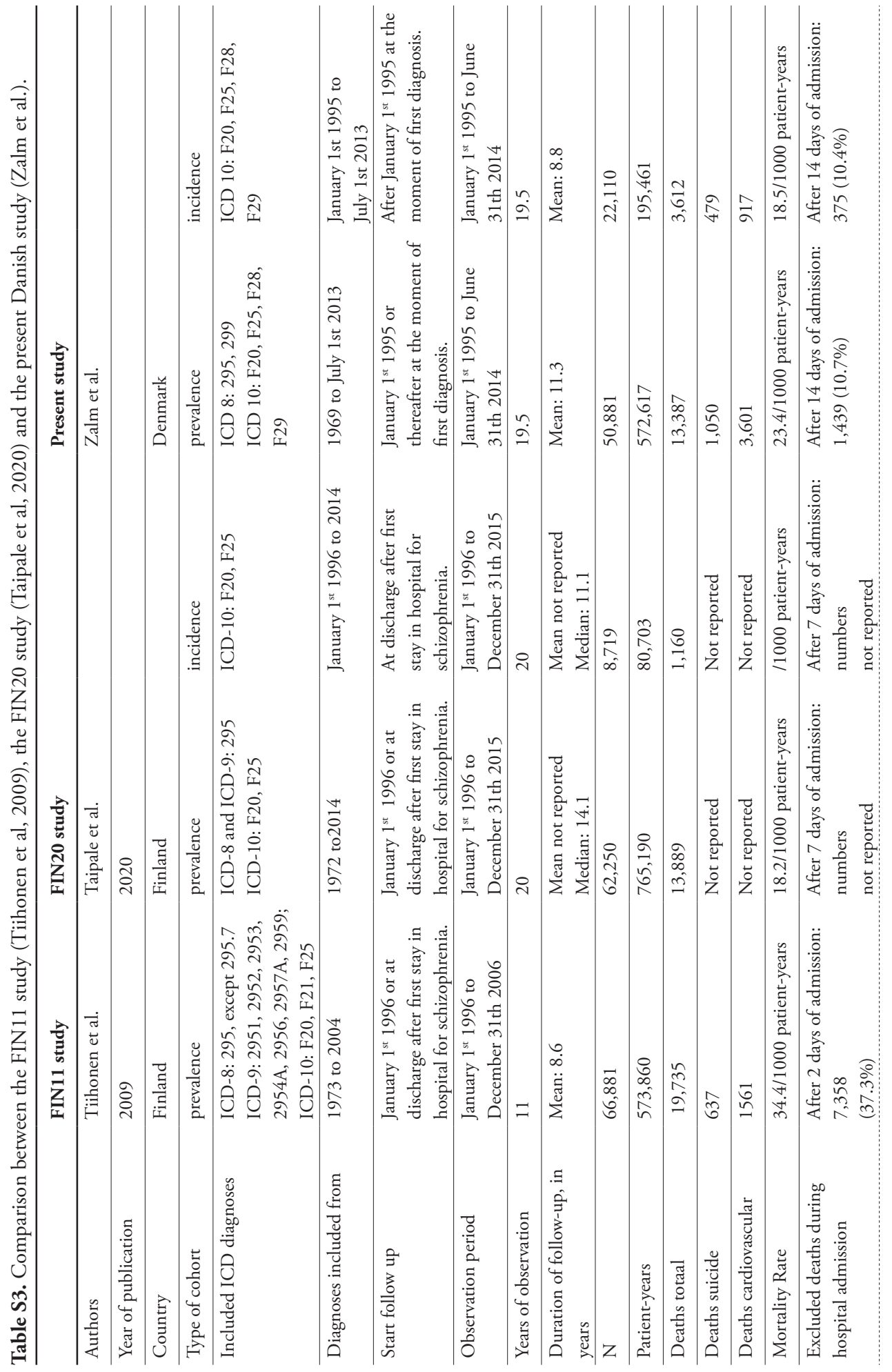




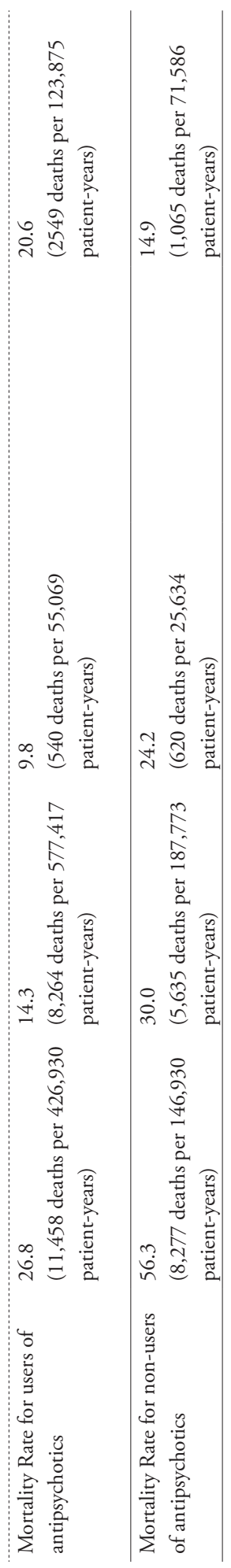




\section{Appendix 1, extra information (in bold) on materials and methods.}

\section{Outcomes}

The primary outcome was all-cause mortality. Secondary outcomes were suicide (ICD10 codes X60-84 and Y10-34) and cardiovascular mortality (ICD-10 codes G45-46 and I10-99).

\section{Exposure}

Treatment was categorized as follows: 1) clozapine (reference); 2) olanzapine; 3) risperidone; 4) other Second-Generation Antipsychotics (SGAs: sertindole, ziprasidone, lurasidone, quetiapine, asenapine, amisulpride, aripiprazole, paliperidone); 5) First Generation Antipsychotics (FGAs: chlorpromazine, levomepromazin, promazine, acepromazine, fluphenazine, perphenazine, prochlorperazine, periciazine, thiordazine, pipotiazine, haloperidol, melperone, pipamperone, bromperidol, flupentixol, chlorprothixene, tiotixene, zuclopenthixol, pimozide, penfluridol, loxapine, sulpiride); 6) polypharmacy including clozapine (polypharmacy is defined as prescriptions for 2 or more different antipsychotics in the same period); 7) polypharmacy not including clozapine; 8) no antipsychotic medication; 9) hospitaldelivered antipsychotic, type unknown: antipsychotics are distributed free of charge to patients sentenced to treatment and, since 2008, during the first 2 years subsequent to a diagnosis of schizophrenia. The type of antipsychotic is not known because the drug is not registered in the prescription registry. A small proportion of these patients will not be using any antipsychotic; 10) Drug Unknown: no data available because of hospitalization, inpatient drug use is not registered in the prescription registry. Episodes of drug use were censored on day 15 of hospitalization. We chose this time period because antipsychotic drugs are often continued at the start of a hospitalization and because their effects are likely to last during this period; 11) no use of antipsychotics.

To define periods of antipsychotic use, we assumed that antipsychotic drug A was used from the moment of redemption for a duration of $\mathbf{9 0}$ days unless: a) a new prescription of the same drug was redeemed; b) another antipsychotic drug $\mathrm{B}$ was prescribed in the 90-days period without a subsequent or concurrently new prescription of drug $A$ before the end of the 90 days period. A period of polypharmacy began when drug $B$ was prescribed during the 90-days period of drug $A$, while drug $A$ was prescribed again after the start of drug $B$. The use of one category of antipsychotic medication for a period of less than 3 months is one episode. If an antipsychotic drug is used for more than 3 months, each period of 3 months constitutes one episode. Episodes of drug use were censored on day 15 of a hospitalization, because during hospital stay no information on drug use is 
available. We took this arbitrary decision, because antipsychotic drugs are often continued during the first two weeks of a hospitalization and because their effects are likely to last during this period.

We conducted separate analyses for current and cumulative use of antipsychotics. Both current and cumulative use are time-dependent variables and were recalculated at the time of each death event in the cohort, both for the patient who died and for those who were still alive at that time. The currently used antipsychotic was defined as the last drug that was prescribed before a death in the cohort, provided that death occurred after no more than 2 weeks of no use or no more than 2 weeks after hospital admission. Cumulative use was defined as a time-dependent variable as well and was recalculated at the time of each death in the cohort. For this measure, all episodes of use of a certain antipsychotic were aggregated and the total duration of these episodes was categorized as follows: $0-1,1-3,3-6,6-10$ years, and more than 10 years. Thus, one individual could contribute to several monotherapy or polypharmacy categories at different points in time during follow-up. However, when a death occurred, a subject was placed in only one category of cumulative antipsychotic use, namely, in the category of the drug that had been used the longest at that time. This implies that shorter periods of use of other antipsychotics at this point in time were disregarded. To illustrate this, after consecutively 2 years of olanzapine, 4 years of clozapine and 3 months of risperidone use, the patient is in the category "risperidone" in the analysis of current use and in the category "clozapine (3-6years)" in the cumulative use analysis. After 3 months of risperidone, 9 months of olanzapine and 6 months of clozapine use, a patient is in the "clozapine" category in the analysis of current use and in the category "olanzapine (0-1 year)" in the analysis of cumulative use. Hazard ratios were calculated with the category clozapine use as reference.

\section{Covariates}

Baseline variables were age at start of follow-up, sex, primary psychiatric diagnosis (ICD10 codes F20, F25, F28 and F29, ICD-8 codes 295 and 299; the first diagnosis was used as the patient's primary diagnosis unless it was later changed into an NAPD diagnosis ranking higher in the following hierarchy: 295/F20 was higher than F25, F25 was higher than 299, 299 was higher than F28 and F28 was higher than F29), and psychiatric hospitalization before follow-up (yes/no). We included the latter variable as a measure of the severity of illness. Duration of illness, i.e., duration since first registered diagnosis of NAPD at the time of cohort entry, was another baseline variable for members of the prevalence cohort. Time-dependent variables were substance use disorder (ICD-8 codes 291, 303, 304; ICD-10 codes F10-F19), drugs for substance use disorder (ATC codes N07B, excluding N07BA), mood disorder (ICD-8 codes 296, 298.09; ICD-10 codes F30-F39), use of antidepressants (ATC codes N06A), 
cardiovascular disorder (ICD-8 codes 400-429, 432-438, ICD-10 codes G45-46, I10-99), drugs for cardiovascular disorders (ATC codes B01, C01, C02, C03, C07, C08, C09 and C10), diabetes (ICD-8 code 250, ICD-10 codes E10-14), drugs for diabetes (ATC codes A10A, A10B) and cancer (ICD-8 codes 140-207, ICD-10 codes C00-C97). The time-dependent variables changed at the time of their first occurrence and were time-lasting (permanent). To illustrate this point, after a diagnosis of a mood disorder or the dispension of a drug for cardiovascular problems, this variable remained 'yes' for the rest of the follow-up period.

\section{Statistical analysis}

For the main analyses, we used an incidence cohort, because follow-up can be started at the moment of the first registration of a diagnosis of NAPD. The analyses for cumulative use were conducted in the incidence cohort only, because we did not have information on the use of antipsychotics before the start of follow-up in the prevalence cohort. Cox proportional hazards regression with time-dependent variables was used to estimate hazard ratios and 95\% confidence intervals (CIs) for the association between exposure to antipsychotics and mortality (all-cause and cause-specific). All subjects were followed up from their first diagnosis, their 15th birthday, or from 1 January 1995, whichever occurred last, until death or 1 July 2014. To allow for the possibility of at least 1 year of follow-up, the latest entry date was 30 June 2013. Right censoring was applied at death, emigration, other disappearance from the Danish centralized civil registration system, the 100 years birthday or the latest on July 1, 2014. Interval censoring was used for persons that emigrated and immigrated again within the study period. Due to violation of the proportional hazards assumption, the Cox analyses were stratified by age at start of follow-up (groups: 0-25, 25-35, 35-45, 45-55, 55-65, 65-75, 75+ years; the groups were merged as necessary), sex, Drug Unknown, and in the prevalence cohort also by (registered) duration of illness before the start of the follow-up (0, 0-5, 5-10, 10-15, 15-20, and 20+ years). The proportional hazards assumption for the Cox regression models was tested and evaluated by graphical assessment of smoothed hazard estimates plots. Clozapine monotherapy was used as reference. The analyses were performed with Stata. A two-tailed p-value of $<0.05$ was considered statistically significant for all tests. Both the analyses of current use and the analyses of cumulative use were conducted for three types of mortality: (1) all-cause mortality; (2) mortality due to suicide; and (3) cardiovascular mortality.

We used two different types of adjustment in order to test the hypothesis that somatic comorbidity and the treatment thereof may influence the association between clozapine and mortality. In the first model, the results were adjusted for the time-fixed variables age at entry, sex, type of NAPD, and psychiatric hospitalization before start of follow-up, and for the time-dependent variables mood disorder, substance use disorder, malignant 
neoplasms, drugs for mood disorder, and drugs for substance use disorder. We adjusted for type of NAPD and psychiatric hospitalization, because they are proxies for illness severity. We adjusted for malignant neoplasms, in order to make sure that any difference between antipsychotics was not due to the occurrence of neoplasms. In the prevalence cohort, we also adjusted for time since first (registered) NAPD diagnosis. In the second model, the results were also adjusted for cardiovascular and diabetic comorbidity (diagnosis and dispension of drugs) as time-dependent variables. 
Figure S1. Adjusted hazard ratios for mortality due to suicide after cumulative use of antipsychotics compared to cumulative use of clozapine (reference), between January 1995 and July 2014, in a Danish incidence cohort of patients with a non-affective psychotic disorder. Comparisons with clozapine were done within each of the groups distinguished by the same length of antipsychotic use: 0-1, 1-3, 3-6, 6-10 years, and more than 10 years. The observation period was from January 1995 to July 2014.

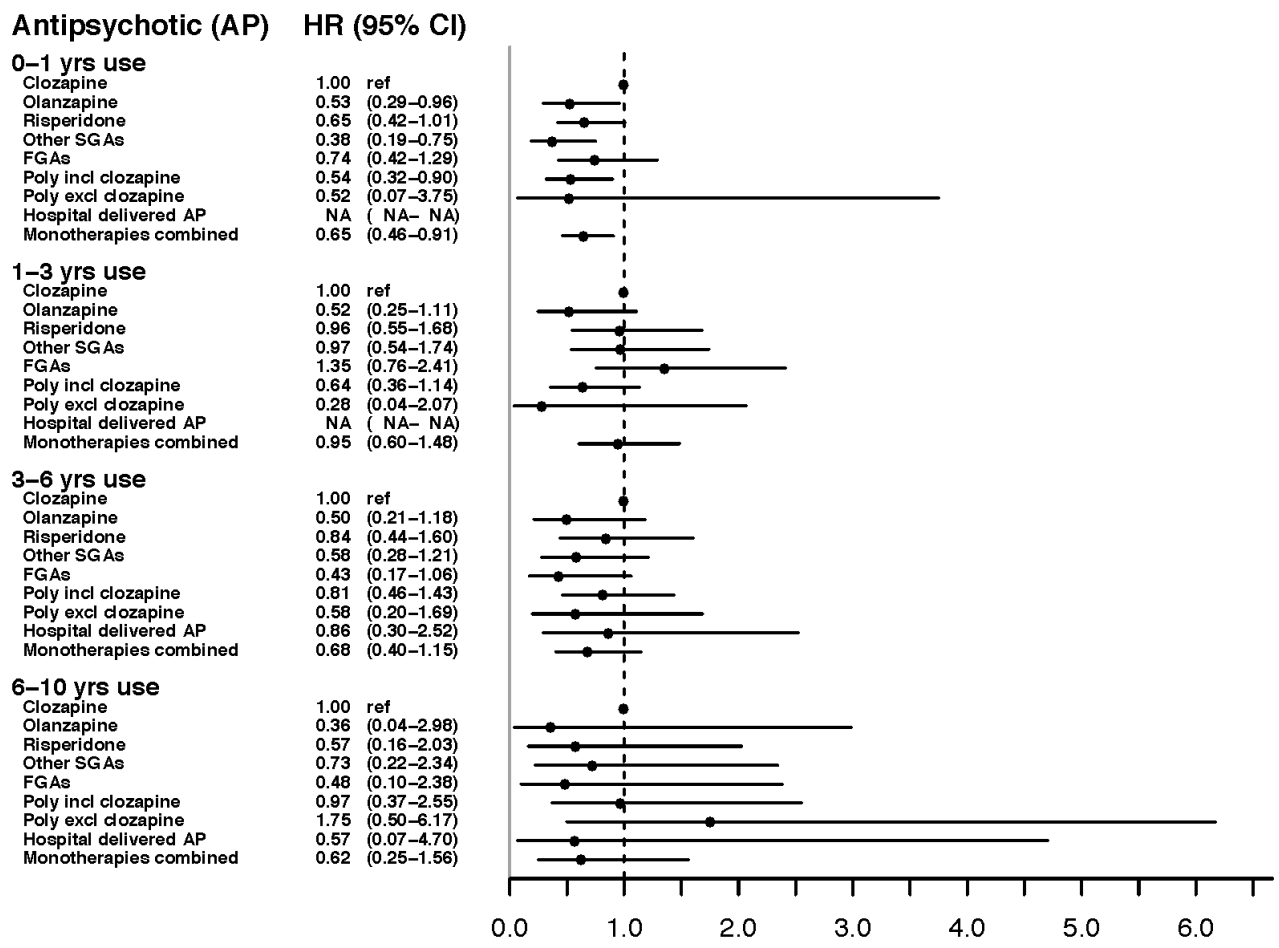

Adjusted for age, sex, type of non-affective psychotic disorder, mood disorder, substance use disorder, psychiatric hospitalization, somatic comorbidity and the treatment of somatic disorders. 
Figure S2. Adjusted hazard ratios for cardiovascular mortality after cumulative use of antipsychotics compared to cumulative use of clozapine (reference), between January 1995 and July 2014, in a Danish incidence cohort of patients with a non-affective psychotic disorder. Comparisons with clozapine were done within each of the groups distinguished by the same length of antipsychotic use: 0-1, 1-3, 3-6, 6-10 years, and more than 10 years. The observation period was from January 1995 to July 2014.

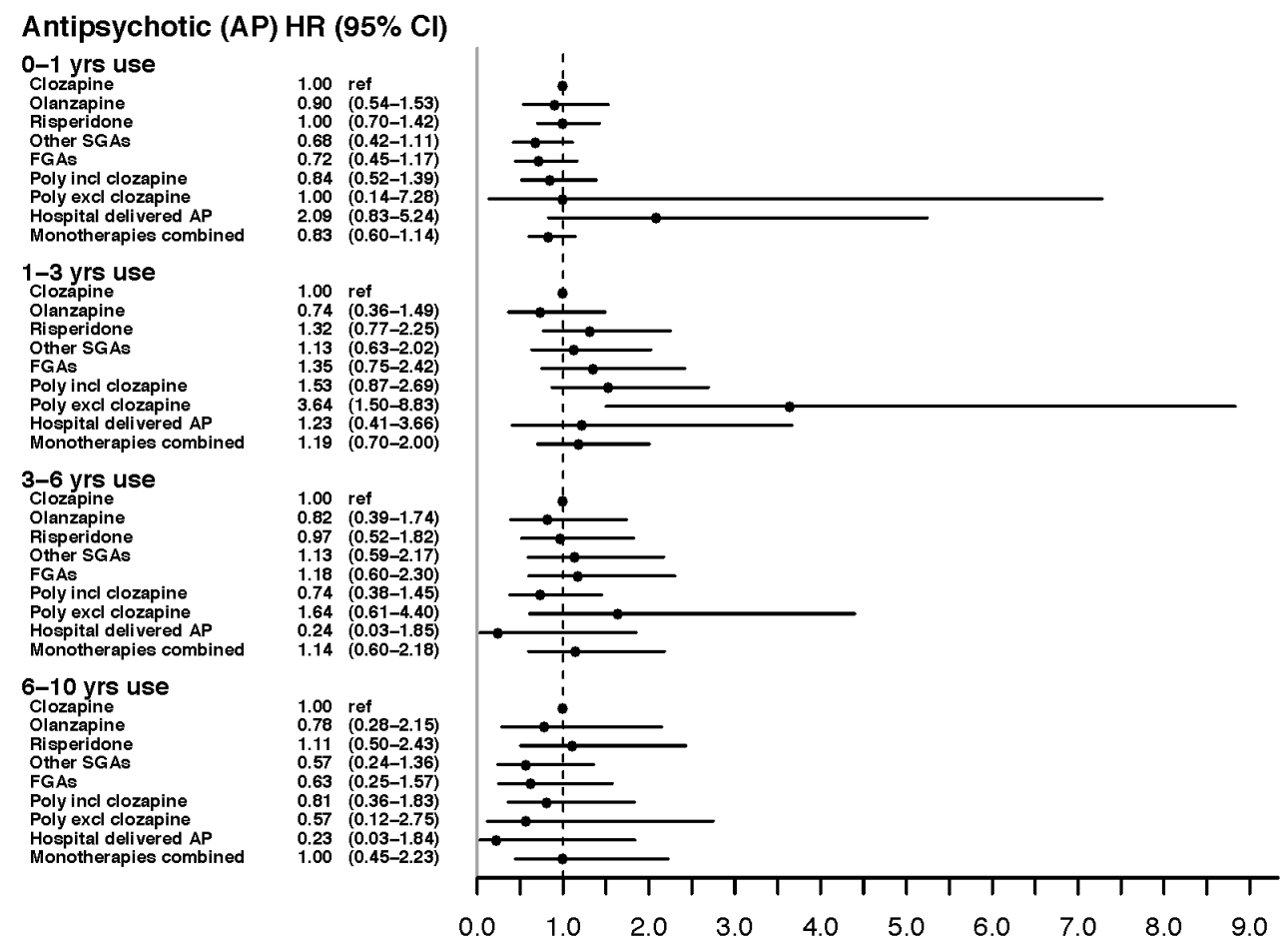

Adjusted for age, sex, type of non-affective psychotic disorder, mood disorder, substance use disorder, psychiatric hospitalization, somatic comorbidity and the treatment of somatic disorders. 
PART III

General discussion and

Addendum 

Chapter 7 
In this last chapter, the findings of this dissertation will be summarized and discussed. First, the research aims and main findings reported in each chapter are described, followed by the discussion of the results, the methodological considerations and the recommendations for future research as well as for clinical practice. At last, conclusions are presented.

\section{Main findings}

The aim of the first part of this thesis was to determine clozapine prescription rates in Dutch ambulatory care and to study the effect of an intervention to improve these rates. For this purpose, we set up a cluster-randomized controlled trial in Flexible Assertive Community Treatment (FACT)-teams and early intervention psychosis (EIP)-teams. In the intervention condition, the psychiatrist delegated the clozapine monitoring tasks to an Advanced Nurse Practitioner (ANP), in the treatment-as-usual condition, he performed the monitoring tasks himself. First, in chapter 2, we presented the baseline characteristics of the patients with Non-Affective Psychotic Disorder (NAPD) treated by 20 FACT-teams and 3 EIP-teams that cooperated in this trial. In the FACT-teams, we assessed 2,286 NAPD patients and found that 23.0\% (N=526) was using clozapine. The differences between teams (range: 8.8 to $34.7 \%$ ) were large and statistically significant. The proportion of patients with an unfulfilled indication for clozapine was $6.9 \%$ (range 1.7-15.6\%; $\mathrm{N}=158$ ). Obviously, in the EIP-teams, these proportions were smaller: $11.3 \%$ and $4.6 \%$ respectively. Overall, the proportion of patients using clozapine was higher than expected and the extent of under-prescription was lower than expected. However, the differences between teams were remarkable. In chapter 3, we tested the hypotheses that psychiatrists would prescribe clozapine more often when they could delegate clozapine monitoring tasks to an ANP, that monitoring by an ANP is at least as safe as monitoring by a psychiatrist and that delegation of monitoring tasks to an ANP is associated with less premature termination of clozapine use. At baseline, there were 173 patients with an unfulfilled indication for clozapine in the FACT and the EIP-teams. Only $6.4 \%$ of these patients started to use clozapine during the inclusion period, 8.5\% in the intervention condition and $4.4 \%$ in the treatment-as-usual condition. Although psychiatrists tended to prescribe clozapine more often if an ANP was available for the monitoring tasks, this result was not significant $(\mathrm{p}=.23)$. Given the low numbers of patients who started to use clozapine, possibly there was a lack of power. Apart from the patients with an indication at baseline, there were also patients newly referred to the teams who started to use this drug and could be included in the other analyses. We found that clozapine-monitoring performed by an ANP seemed at least as safe as that done by a psychiatrist and there seemed to be no differences in premature termination of clozapine. 
In the second part of this thesis we aimed to clarify the association between clozapine and mortality. Therefore, we first commented on several studies on the association between clozapine and mortality in chapter 4 . In this comment, we discussed the biases and methodological errors in studies that reported a significantly decreased mortality associated with the use of clozapine. The results of these studies may have been influenced by, for example, survivorship bias, immortality bias and the lack of adjustment for age. We showed that a meta-analysis of this topic was flawed, because it used data unadjusted for age (Vermeulen et al., 2019).

In chapter 5 we aimed to study other possible sources of bias in studies on clozapine and mortality. We tested the hypothesis that clozapine may be prescribed more often to relatively healthy patients. This mechanism, known as confounding by indication, may explain to some extent the reports of a lower mortality associated with clozapine. Another hypothesis implied that intensive monitoring during clozapine use may prevent (under-treatment of) somatic disorder, indirectly resulting in a decreased mortality among clozapine-users. In this pharmaco-epidemiological study, we showed that patients who started with clozapine were suffering from at least as much cardiovascular or diabetic comorbidity as the patients starting with other antipsychotics, suggesting that confounding by indication is not a likely explanation for a presumed lower mortality in clozapine users. However, during the use of clozapine new cardiovascular or antidiabetic drugs were prescribed earlier and more often, compared to those using olanzapine. This suggests that those using clozapine may receive more adequate somatic treatment which in turn could help decrease mortality.

In chapter 6 we analyzed the association between clozapine and mortality with the use of Danish National registers, including 22,110 patients with a first diagnosis of NAPD (incidence cohort) and 50,881 patients ever diagnosed (prevalence cohort). The maximum observation time in this study was 19.5 years. We examined whether mortality (all-cause and cause-specific) during current use of clozapine was lower than mortality during current use of other antipsychotics. Our second aim was to assess whether different results are obtained when including a measure of cumulative use. For the calculation of exposure to cumulative use at a certain time point, earlier episodes of use were taken into account. For each patient, all episodes of antipsychotic use were aggregated and categorized as follows: use during 0-1, 1-3, 3-6, 6-10 years, and more than 10 years. In the analysis, at each time point, a patient was placed in the category of the drug that had been used the longest at that time. This necessarily implies that shorter periods of use of other antipsychotics at this point in time were disregarded, but this prevents that patients are put in more exposure categories at the same time and, as a consequence, are compared to themselves. In general, we found no evidence of a lower all-cause or cardiovascular mortality associated with current use of clozapine in 
the incidence or prevalence cohort. Mortality due to suicide was lower during the use of clozapine than during the use of all other antipsychotics. However, this difference was non-significant when comparing clozapine with the other categories separately, except for the category "hospital delivered antipsychotics". This latter category consists of patients to whom antipsychotics are distributed free of charge (because they were sentenced to treatment or, since 2008, in the first 2 years subsequent to a diagnosis of schizophrenia). It is unknown whether and which antipsychotics are prescribed to these patients.

Cumulative use of clozapine for 6 years or more was not associated with a higher all-cause or cardiovascular mortality compared to other antipsychotics, despite of its potential metabolic side-effects. The adjustment for the presence and treatment of somatic comorbidity hardly affected the results. However, cumulative use of clozapine between 0 and 1 year, was associated with a higher mortality (all-cause and suicide), compared to almost all other categories. The opposing results of a lower risk of death (all-cause or suicide) during current use of clozapine and a higher risk of death associated with cumulative use of clozapine, suggest that cessation of clozapine marks a period of high risk of all-cause mortality and suicide.

\section{Discussion of main findings}

\section{Clozapine prescription and under-prescription}

The major differences between teams in clozapine prescription rates are consistent with results of other international studies (Nielsen, Roge, Schjerning, Sorensen, \& Taylor, 2012; Stroup, Gerhard, Crystal, Huang, \& Olfson, 2014; Verdoux et al., 2016). However, our result of a mean prescription rate of $23 \%$ was much higher than reported by other studies. Although it is unclear whether the mean prescription rate found in our study was representative for the Netherlands, evidence suggests it is. Following a method used in an Australian study (Malalagama, Bastiampillai, \& Dhillon, 2011), we combined a Dutch national prescription database (GIP / Zorginstituut Nederland (2016) with an assumed schizophrenia prevalence rate. The Australian authors used a point prevalence of schizophrenia (0.46\%) (Saha, Chant, Welham, \& McGrath, 2005) and calculated that an estimated $19 \%$ of all Australian patients with schizophrenia between 18 and 65 years old was using clozapine. When we use the same point prevalence of schizophrenia, which is in line with the prevalence of schizophrenia found in the Dutch Nemesis study (Bijl, Ravelli, \& van Zessen, 1998), the clozapine prescription rate in the Netherlands is approximately $20 \%$. One possible explanation for this high prescription rate in the Netherland is the effort of the Dutch Clozapine Collaboration group, recognized as a national center of expertise for clozapine (Bogers, Schulte, Van 
Dijk, Bakker, \& Cohen, 2016). Since the group was founded in 1998, the number of outpatient clozapine users has nearly tripled from 4,891 to more than 14,064 in 2017 (GIP / Zorginstituut Nederland (2016). The trainings provided by this expert group and the facilitated contact with experienced prescribers have been found to facilitate the initiation of clozapine (Verdoux, Quiles, Bachmann, \& Siskind, 2018). A similar initiative in New York State (Carruthers et al., 2016) also showed its effectiveness in increasing prescription rates. Another explanation may be that there is more attention for clozapine use and guidelines in educational programs in the Netherlands, as trainings by the expert group are incorporated into the national teaching program for residents in psychiatry. More knowledge on clozapine monitoring is likely to increase prescription rates (Tungaraza \& Farooq, 2015).

The assessment of all patients in our study did also result in the exact numbers of unfulfilled indications for clozapine, i.e. the degree of under-prescription of $6.9 \%$. Our results were lower than expected and lower than the $8.5 \%$ found in the UK study by Patel et al. (Patel et al., 2014), the only other study into unfulfilled indications for clozapine. However, the criteria of unfulfilled indications for clozapine in both studies differed. In the UK all patients treated for more than a year, having substantial symptoms and no clear reason documented as to why they had not had a trial with clozapine, were considered to have an unfulfilled indication for clozapine. In our study there was also a category of patients with an unfulfilled indication for clozapine (8.6\%) who were at least markedly psychotic, but had not yet received two adequate treatments with antipsychotic drugs. One can think of patients who refuse for a long period of time an adequate dosage. Since we do not know which proportion of these patients had been treated for more than a year in the participating teams, it is difficult to compare these numbers to those in the UK.

Our intervention of delegating clozapine-monitoring to an ANP did not result in significantly higher prescription rates. Assessing patients for an indication for clozapine at baseline did not lead to a substantial improvement in prescription rates either, since only $6 \%$ of those with an indication at baseline started to use clozapine during the observation period. Despite the results of previous research indicating that psychiatrists considered the presence of dedicated staff to perform clozapine monitoring as the most helpful (Gee, Vergunst, Howes, \& Taylor, 2014; Goren et al., 2016), our results show no major improvement in prescription rates when monitoring tasks could be delegated. In a recent systematic review (Verdoux et al., 2018) a lack of personal experience and concerns about blood monitoring and adverse effects were found to be important barriers in prescribing clozapine. Prescribers and ANPs participating in our study were offered a voluntary training by experts to increase their knowledge and confidence. While most ANPs were present during this training, it was not attended by the psychiatrists who 
were most reluctant of prescribing clozapine. This suggests that personal preferences are the most important barrier to or facilitator of starting with clozapine and that providing information on monitoring is insufficient for a part of the prescribers. Our finding of a relatively low number of patients who start to use this drug in teams with low clozapine prescription rates and vice versa, supports this idea. It seemed that psychiatrists who were unexperienced or expressed a negative view on clozapine would not prescribe clozapine, regardless of the possibility to delegate monitoring. Prescribers with more experience and a positive attitude had higher prescription rates at baseline and tended to prescribe clozapine more often during the study. Some psychiatrists in our study stated that this intervention allowed them to start with outpatient initiation of clozapine, but to further increase prescription rates, other interventions are needed as well. Although our training was targeted on fears of prescribers, it was not mandatory and therefore not attended by those with a negative view on clozapine. Future interventions may be more successful when they are accompanied by institutional pressure. Another possibility is the integration of clozapine use as a quality criterion in the audits for outpatient care teams. This approach has been shown successful in initiatives from New York (Carruthers et al., 2016) and New Zealand (Wheeler, 2008).

Another possible intervention that may be effective in improving clozapine prescription rates is the use of a point-of-care device (on the spot testing) for total and differential white cell count. Both Nielsen (Nielsen, Thode, et al., 2012) and Bogers (Bogers, Bui, Herruer, \& Cohen, 2015) found that patients experienced the use of such a device as less painful than venous sampling and less inconvenient. Additionally, the method used moderately influenced their motivation for clozapine therapy. Using this method might therefore boost clozapine prescription rates. It is unclear whether the use of a point-of-care device could also diminish the fear of prescribers for the adverse effects of clozapine. However, it is conceivable that the immediate result and the independence from external laboratories increase the sense of control and safety among prescribers.

\section{Clozapine monitoring}

In the Netherlands, without a manufacturer-organised service or database for haematological monitoring (leukocyte and neutrophil counts), it is the responsibility of the physician to organize the weekly laboratory exams from the start of the use of clozapine. We did not expect that only about $70 \%$ of the mandatory White Blood Cell (WBC)-counts were performed and we do not know whether this result is representative for the Dutch practice. However, psychiatrists and ANPs were aware of the aim of our study on safety of monitoring and it is likely that they would rather perform better than worse during the study. The training on clozapine monitoring preceding the trial increased the knowledge of the ANPs and psychiatrists and is also likely to have positively affected the proportion of WBC-counts performed in our study. Considering 
the large differences we found between teams and the small difference between the 2 conditions, we deem it likely that local practices and personal factors such as experience and punctuality might influence the number of white blood cell counts performed. Although many European countries do not have a mandatory monitoring system (which means that reimbursement of clozapine is not conditional on checking the WBC-counts), there is only one study that reported the frequency of WBC-counts (Ingimarsson, MacCabe, Haraldsson, Jonsdottir, \& Sigurdsson, 2016). This study was conducted in Iceland where, in contrast to the European Union, even the WBC-counts are not mandatory according to the clozapine registration text. Consequently, the mean interval of 25 days between WBC-counts during the first 18 weeks of clozapine use was considerably longer than found in our study. It is unknown whether this lack of mandatory regulations affects prescription rates.

Side-effects of antipsychotics can be as distressing as psychotic symptoms. Considering clozapine use, side-effects can be burdensome, especially during the initiation phase. Since starting clozapine as an outpatient is a predictor for premature termination (BeexOosterhuis, Heerdink, Van Gool, \& van Marum, 2018), optimal treatment of sideeffects may result in less premature termination and help more patients experience the benefits of clozapine treatment (Legge et al., 2016). We expected that our intervention could contribute to better treatment of side-effects, because ANPs might be able to maintain a better therapeutic alliance and have more time for querying side-effects (Sin $\&$ Gamble, 2003). However, the difference in premature termination we found was small and not significant.

\section{Clozapine and mortality All-cause mortality}

Previous studies on clozapine and mortality obtained mixed results. Several studies reported a decreased mortality, where other studies found no differences between clozapine and other antipsychotics. In general, it is difficult to compare studies on clozapine and mortality because of differences in outcome, inclusion criteria, exposure (e.g. continuous use of an antipsychotic vs. intention-to-treat), comparison groups and adjustments. A meta-analysis of all those studies in different countries would be desirable, but as we described in chapter 4, with the existing studies on clozapine and mortality it is difficult to perform such an analysis. Apart from these methodological differences, there are other causes for obtaining different results in different countries. For example, there may be differences in prescription rates. A more restrictive use of clozapine implicates that only the most severely ill patients with the shortest lifeexpectancy start with clozapine. Another possible cause for different findings in different countries, may be differences in indications for clozapine (indicated for suicidal patients or not). 
In studies in the same country with a similar methodology, similar results are expected, but two Finnish studies show that even then inexplicable differences in results are possible. The authors of the FIN11 study (Tiihonen et al., 2009), published in The Lancet, conducted a new Finnish study with a longer observation period, the FIN20 study (Taipale et al., 2020). The results of the latter study differed from the results of the FIN11 study in that the mortality during clozapine use was no longer significantly lower than during the use of all other categories of antipsychotics. One possible explanation for the difference in the results is the exclusion of deaths after 2 days of hospitalization in the FIN1 1 study compared to 7 days in the FIN20 study. (The authors had to set a limit, because they had no information on medication use during hospital admission.) Besides this difference there must be other differences between the FIN11 and the FIN20 study that are unclear. The inclusion criteria in both Finnish studies were similar (the only difference was that patients with ICD-10: F21, schizotypal disorder, were included in FIN11 and not in FIN20) and the observation periods started at the same date. However, more patients were included in the study with the shorter observation period and the mortality rate was also much higher in the FIN11 study, compared to the FIN20 study. It seems that the authors of the FIN-20 study wanted to correct mistakes committed in their FIN-11 study, but this is uncertain.

The aim of our Danish study on clozapine and mortality was to conduct a study similar to the FIN11 study (Tiihonen et al., 2009), but with the use of an incidence cohort to avoid survivorship bias and with adjustment for somatic comorbidity. Additionally, we wanted to include almost all deaths in the analysis, so we excluded deaths after 14 days of hospitalization instead of 2 days as was done in the FIN1 1 study.

Our finding that clozapine is not associated with a lower all-cause mortality differs from the FIN11 study (Tiihonen et al., 2009), but is consistent with several other large database studies (Ringback Weitoft et al., 2014; Stroup, Gerhard, Crystal, Huang, \& Olfson, 2016; Taipale et al., 2017). Authors of these studies have tried to explain the differences between their study and the FIN11 study. Authors from a Swedish study (Ringback Weitoft et al., 2014) suggested for example that the differences between their results and those of the FIN11 study are due to longer observation period in the FIN11 study (11 years compared to 4 years in the Swedish study). However, our study had an observation period of maximum 19.5 years and its results are more in line with the Swedish study, showing that duration of observation period does not explain the difference. This is supported by the recently published FIN20 study (Taipale et al., 2020), which had a longer observation period and nevertheless reported no significant differences in mortality between clozapine use and the use of other antipsychotics. The authors of another Swedish study (Taipale et al., 2017) presumed that clozapine is only prescribed to the most severely ill patients in Sweden in contrast to a more frequent use 
in Finland (Taipale et al., 2017). The most severely ill are supposed to have a shorter life-expectancy, thus explaining the difference in results between the Swedish and the FIN-11study. The FIN20 study shows otherwise, as clozapine is not associated with a significantly decreased mortality as it was in the FIN11 study.

Another possible explanation is the exclusion of $64 \%$ of deaths in the FIN11 study (those who were admitted to hospital longer than 2 days). This may have affected the results. In the Swedish study (Taipale et al., 2017) all deaths were included, in the FIN20 study (Taipale et al., 2020) deaths were censored after 7 days of hospital admission and in our Danish study, deaths were censored after 14 days of hospital admission. Since clozapine use is associated with urgent hospitalization for physical health problems (Vanasse et al., 2016), the results of the FIN11 study (Tiihonen et al., 2009) may have been an underestimation of the mortality associated with clozapine use if these deaths occurred after the second day of hospitalization. We aimed to conduct a study with a better methodology than that of the FIN11 study and compared the results obtained from an incidence cohort to those from a prevalence cohort and adjusted the results for somatic comorbidity and the treatment thereof.

\section{Mortality due to suicide}

When analyzing the association between clozapine use and suicide, it is important to account for survivorship bias. Suicides occur more frequently in the first years after the onset of psychosis (Kuo, Tsai, Lo, Wang, \& Chen, 2005; Melle et al., 2017; Termorshuizen et al., 2013), whereas clozapine is often first prescribed several years after onset (Howes et al., 2012; Nielsen, Roge, et al., 2012). In the FIN20 study (Taipale et al., 2020) a sensitivity analysis was performed, excluding the first 10 years of followup. As the results for clozapine were less beneficial in the sensitivity analysis, this study shows that survivorship bias may affect the observed association between clozapine use and suicide. Although survivorship bias may have caused an underestimation of suicides during clozapine use in various studies, our results of a non-significantly lower risk of suicide during clozapine use in the incidence part of the Danish cohort contribute to the existing evidence that the risk of suicide is lower during clozapine use. This may be due to the effectiveness of this drug, reducing psychosis and anxiety. Another explanation may be that clozapine is associated with good drug adherence (Cooper, Moisan, \& Gregoire, 2007) and good drug adherence is associated with a lower risk of suicide (Ringback Weitoft et al., 2014). It was not possible to test this hypothesis with a database containing dispensed medication without information on actual use.

Of note, the lower risk of suicide found in our study must be seen in the light of numbers of suicides expected during the use of clozapine. Although there were no suicides among members of the incidence cohort who used clozapine in the first 6 years of follow-up, the 
expected number of suicides would have been 4, based on person years of clozapine use and risk of suicide among users of other antipsychotics. This number shows that even in a large database study like ours ( 7 million inhabitants, a mean clozapine prescription rate of $10 \%$ and an observation period of 19.5 years), the number of suicides is too small to perform useful statistical analyses and find a significant difference in number of suicides. Still, absence of any suicide event during the early years after the diagnosis indicates that prescribing clozapine in an early phase after onset of schizophrenia may be of importance when aiming to prevent suicides.

The main aim of our analyses of cumulative use were to study the effect of long-term use of clozapine on cardiovascular mortality. Surprisingly, we found a higher overall mortality and especially a higher mortality due to suicide among patients in the category of cumulative clozapine use up to 1 year, compared to other antipsychotics. (Cumulative use of a certain antipsychotic up to 1 year at a certain time point means that the patient has used that antipsychotic for a longer period of time than any other antipsychotic, but no longer than 1 year at that time point.) This increased suicide mortality, in combination with the lower risk of suicide during current use of clozapine strongly suggests that most of these suicides occurred after termination of clozapine use. This finding is in line with the Danish results found by Wimberley et al., who report an increased hazard of death in periods after clozapine discontinuation compared to periods of clozapine use (HR: 2.65; 95\% CI: 1.47-4.78) (Wimberley et al., 2017). The authors of this study assume that the excess mortality rate in the first year or even within 3 months after discontinuation is due to causes other than suicide. However, our data show that the increased mortality risk after termination of clozapine is most prominent in the analysis of suicide. In 1999, Walker et al. (Walker, Lanza, Arellano, \& Rothman, 1997) already reported an increased suicide mortality after stopping clozapine use compared to this mortality during its use and interpreted this as evidence of a protective effect of clozapine. Patchan et al. (Patchan, Richardson, Vyas, \& Kelly, 2015) described the increased suicide risk after termination of clozapine in 3 case studies. Considering that clozapine is a drug of last resort, ending its use means going back to a non-effective other drug, likely to lead to relapse. A relapse could also be caused by acute clozapine withdrawal as there is stronger evidence for a rapid-onset psychosis (supersensitivity psychosis) following clozapine withdrawal than following withdrawal of other antipsychotic drugs (Moncrieff, 2006). In addition, there may be a psychological argument, as quitting clozapine use may lead to despair since clozapine often is introduced as "medicine of last resort". Our findings support the hypothesis that there is not only a protective effect of clozapine, but also an increased risk of mortality in general and suicide in particular, after termination of clozapine compared to other antipsychotics. 


\section{Cardiovascular mortality}

Given the weight gain and negative influence on other metabolic parameters associated with clozapine use, one would expect an increased cardiovascular mortality. We hypothesized that (1) confounding by indication and (2) better somatic monitoring could have affected the association between clozapine and cardiovascular mortality in studies that reported a decreased mortality associated with clozapine.

With reference to our first hypothesis: in our Dutch database study in chapter 5, about the physical health of patients being prescribed clozapine or using clozapine, we observed that clozapine was not prescribed to (on average) healthier patients. These results are in line with a study in Denmark, that reported no differences between starters with clozapine and starters with other antipsychotics on the mean comorbidity index. In a study in Canada (Vanasse et al., 2016), on the contrary, starters of clozapine were found in better health. This could be explained by the more restrictive use of clozapine in Canada, possibly leading to confounding by indication (only prescribing clozapine to those who are healthy). The high prescription rates in the Netherlands reported in chapter 2, combined with the findings in chapter 5 are supportive of the idea that there is less confounding by indication in countries with more extensive use. The results of our Danish study in chapter 6 also support this idea because adjustments for somatic comorbidity and treatment thereof hardly affected the results. It was impossible to test this hypothesis in the Danish data.

With reference to our second hypothesis: patients prescribed clozapine have more contact with clinicians than others (Hayes et al., 2015). However, the effect of more contact on physical health in patients using clozapine had not been studied yet. In our Dutch pharmaco-epidemiological study we found differences in somatic treatment between users of clozapine and users of olanzapine that were most prominent for oral antidiabetics, 'lipid modifying agents' and 'beta blocking agents'. Because we compared clozapine-users to users of olanzapine, which has a similar profile of side-effects, we concluded that patients using clozapine are better monitored and receiving better somatic treatment. We presume that the frequent blood monitoring and face-to-face contact (especially in the initiation phase), in combination with the fear of adverse sideeffects, causes alertness and induces better somatic treatment. However, this hypothesis was not supported by the results of our Danish study, because adjusting for somatic comorbidity and the treatment thereof hardly affected the association with mortality.

To study the association between cardiovascular mortality and clozapine, current clozapine use is not a good measure given the long-term (metabolic) effects of clozapine. In addition, as Walker et al. already suggested in 1999, it is likely that moribund patients are taken off clozapine and that its use is terminated as soon as cardiovascular 
problems are emerging. Therefore, to study the effect of long-term use of clozapine on cardiovascular mortality, a measure of cumulative use should be used. The only other study on cumulative use of antipsychotics and mortality is the FIN11 study (Tiihonen et al., 2009), but the results of this study are not comparable to those of our study, due to very different analyses. Instead of the prevalence cohort used in the FIN11 study, we analyzed an incidence cohort with cumulative use as a time-dependent variable.

It is true, previous studies concluded that clozapine use does not increase cardiovascular mortality, but our study is the first study to confirm this using an incidence cohort. Remarkably, cumulative use of clozapine of more than 6 years does not seem to increase cardiovascular mortality, despite of its known metabolic side-effects. This is in line with the findings of Kelly et al. (Kelly et al., 2010) who reported no significant differences in cardiovascular mortality between clozapine-users and risperidone-users after a 6-10 year observation period. This is also in accordance with the findings of the FIN20 study, that reported no differences in all-cause and cardiovascular mortality between cumulative use of clozapine and cumulative use of other antipsychotics (Taipale et al., 2020). Several explanations are possible. One of them is a decrease in smoking during clozapine use (Machielsen et al., 2012; McEvoy, Freudenreich, \& Wilson, 1999). Another possible explanation is the effectiveness of clozapine on psychotic symptoms, which may result in a major reduction of the stress level and an increased ability to use health care facilities and seek help for physical symptoms.

\section{Methodological considerations}

This thesis used various designs to answer the research questions. The cross-sectional design in the study in chapter 2 together with the cluster-randomized trial described in chapter 3 provides a complete overview of prescription rates, under-prescription and new prescriptions of clozapine. However, the selection of the teams was not random in each participating institute. At one institute, where all teams with an ANP were expected to participate, the differences in prescription rates were large. The teams from the other 3 institutes volunteered to participate after a call to join this study. The psychiatrists and ANPs of these latter teams may have had a positive view on clozapine, which may have affected the prescription rates at baseline described in chapter 2 and the results of the trial described in chapter 3. However, we did not study the characteristics of prescribers. Before the observation period there were many changes in prescribers and their predecessors may have been responsible for high or low rates at baseline. During the observation period there were many changes in staff too. 
Another limitation of the studies is the generalizability to other countries. Although multidisciplinary outpatient teams like those in the Netherlands are comparable to services in other European countries (Rosenheck et al., 2016; Valdes-Stauber, Putzhammer, \& Kilian, 2014), monitoring guidelines outside the European Union may differ.

The chapters 5 and 6, about clozapine and mortality, used large Dutch and Danish databases. In general, important information is lacking in such studies. For example, drug prescription databases only contain information on reimbursed drugs, while it is unknown whether the patient is actually using the drugs. In addition, drug use during hospital stay was not registered in the prescription databases. In both chapters, information on dosage was unreliable and could not be used. Another limitation was the absence of information on disease severity. In the Danish study, we used psychiatric hospitalization (yes/no) as a measure of severity of disease. We could have chosen to use the number of hospitalizations or total duration of hospitalizations as a proxy of disease severity, but due to the large number of variables already included we chose not to do so. Information on lifestyle (e.g., smoking) was lacking too. Since the insurance database used for chapter 5 contained information up to 5 years, information regarding the period before follow-up was absent, as was information on variables like duration of illness and reasons for prescribing clozapine.

\section{Implications for clinical practice and future research}

We conducted the first randomized trial world-wide to examine the effectiveness of an intervention to stimulate the prescription of clozapine. Delegating clozapine monitoring tasks to an ANP did not result in a substantial increase in the clozapine prescription rates, but proved to be safe. Since we also assessed patients for an indication for clozapine at baseline, we know that this assessment did not contribute to a substantial increase in prescription rates either. However, the likelihood of a significant effect may have been reduced because the prescription rates were already very high. In other countries, with lower prescription rates or fewer psychiatrists, our intervention might have been very effective. It's worth to repeat this trial in such a country and to examine whether training ANP's may result in better treatment for severely ill patients.

Examples of other interventions that can be studied are the use of a point-of-care device (Bui, Bogers, Cohen, Njo, \& Herruer, 2016), integrated clozapine community clinics (Clark, Wilton, Baune, Procter, \& Hustig, 2014) and clozapine community initiation teams (Beck et al., 2014). Another target in research on improving prescription rates is a change in the psychiatrist's attitude towards clozapine and a reduction of his 'prescribers 
fear'. Extra training and courses for psychiatrists may help to reduce this fear and lead to more confidence. Institutional pressure and integration of clozapine use as a quality criterion in the audits for outpatient care teams, might be needed too (Verdoux et al., 2018). Continuing research on this subject is important, because starting clozapine or a shorter delay before starting clozapine can lead to earlier recovery from chronic psychosis and better patient outcome (Ucok et al., 2015).

For clinical practice there are four important conclusions about clozapine and mortality that can be drawn from this thesis. First, we showed that it is unlikely that clozapine use is associated with a decreased all-cause mortality. The conclusions of a meta-analysis on this topic (Vermeulen et al., 2019) were misleading, as is the editorial by John Kane in the American Journal of Psychiatry (Kane, 2017) with the headline 'clozapine reduces all-cause mortality'. This statement is based on a single study (Wimberley et al., 2017) in which patients with TRS using clozapine were compared to TRS patients not using clozapine (including those not using any antipsychotic at all). The second conclusion is that clozapine use was not associated with an increased cardiovascular mortality. Our study is the first study using an incidence cohort for this purpose and with a long observation period. The third conclusion is the decreased risk of suicide during the use of clozapine and the fourth is the increased risk of suicide after stopping clozapine within 1 year.

Since there is no evidence of an increased mortality during clozapine use, not even in cardiovascular mortality, we feel that prescribers should not fear the side-effects of clozapine and not be hesitant to prescribe it. Apart from the superiority of clozapine in treatment resistant schizophrenia, the growing body of evidence concerning the lower risk of suicide during clozapine use should also be a reason to prescribe clozapine sooner and more often if other antipsychotics are not effective.

An important finding that requires the attention of the clinician and further research is the increased risk of mortality in general and suicide in particular after termination of clozapine within the first year. Carefully tapering of clozapine dosage is important to prevent relapse even if clozapine had not been effective. After termination of its use, careful monitoring of psychosis and suicidality is needed for an as yet unknown period of time. If clozapine use has to stop immediately (due to side-effects like agranulocytosis, ileus or myocarditis), careful monitoring of suicidality is even more important. It is important to gain knowledge on the duration of this increased risk period and about the strength of this association. 


\section{Conclusions}

In this dissertation we have focused on improving prescription rates of clozapine and on the association between clozapine and mortality. Overall, prescription rates in the Netherlands were high and our intervention of delegating monitoring tasks to an ANP did not significantly raise them. In countries with lower prescription rates or lower numbers of psychiatrists, however, this safe intervention could be very useful.

We have shown that several reports of a reduced mortality associated with the use of clozapine, published in influential journals like The Lancet, the American Journal of Psychiatry and Schizophrenia Bulletin were misleading. We found no major differences in all-cause mortality between current or cumulative use of clozapine and current and cumulative use of other antipsychotics. Furthermore, our study adds to an increasing body of evidence supporting a protective effect of clozapine against suicide. However, our results also indicate an increased risk of suicide for those who terminate clozapine use within 1 year. This should be a cause for concern. Finally, even after 19.5 years of observation we found no evidence of an increased risk of cardio-vascular mortality. 


\section{References}

Beck, K., McCutcheon, R., Bloomfield, M. A., Gaughran, F., Reis Marques, T., MacCabe, J., ... Howes, O. D. (2014). The practical management of refractory schizophrenia--the Maudsley Treatment REview and Assessment Team service approach. Acta Psychiatr Scand, 130(6), 427-438. doi:10.1111/ acps. 12327

Beex-Oosterhuis, M. M., Heerdink, E. R. R., Van Gool, A. R., \& van Marum, R. J. (2018). Predicting Unsuccessful Clozapine Treatment After First Use in Adult Patients With Psychotic Disorders. J Clin Psychopharmacol, 38(6), 604-608. doi:10.1097/jcp.0000000000000977

Bijl, R. V., Ravelli, A., \& van Zessen, G. (1998). Prevalence of psychiatric disorder in the general population: results of The Netherlands Mental Health Survey and Incidence Study (NEMESIS). Soc Psychiatry Psychiatr Epidemiol, 33(12), 587-595. doi:10.1007/s001270050098

Bogers, J. P., Bui, H., Herruer, M., \& Cohen, D. (2015). Capillary compared to venous blood sampling in clozapine treatment: patients and healthcare practitioners experiences with a point-of-care device. Eur Neuropsychopharmacol, 25(3), 319-324. doi:10.1016/j.euroneuro.2014.11.022

Bogers, J. P., Schulte, P. F., Van Dijk, D., Bakker, B., \& Cohen, D. (2016). Clozapine Underutilization in the Treatment of Schizophrenia: How Can Clozapine Prescription Rates Be Improved? J Clin Psychopharmacol, 36(2), 109-111. doi:10.1097/jcp.0000000000000478

Bui, H. N., Bogers, J. P., Cohen, D., Njo, T., \& Herruer, M. H. (2016). Evaluation of the performance of a point-of-care method for total and differential white blood cell count in clozapine users. Int J Lab Hematol, 38(6), 703-709. doi:10.1111/ijlh.12561

Carruthers, J., Radigan, M., Erlich, M. D., Gu, G., Wang, R., Frimpong, E. Y., . . Stroup, T. S. (2016). An Initiative to Improve Clozapine Prescribing in New York State. Psychiatr Serv, 67(4), 369-371. doi:10.1176/appi.ps.201500493

Clark, S. R., Wilton, L., Baune, B. T., Procter, N., \& Hustig, H. (2014). A state-wide quality improvement system utilising nurse-led clinics for clozapine management. Australas Psychiatry, 22(3), 254-259. doi:10.1177/1039856214533395

Cooper, D., Moisan, J., \& Gregoire, J. P. (2007). Adherence to atypical antipsychotic treatment among newly treated patients: a population-based study in schizophrenia. J Clin Psychiatry, 68(6), 818825.

Gee, S., Vergunst, F., Howes, O., \& Taylor, D. (2014). Practitioner attitudes to clozapine initiation. Acta Psychiatr Scand, 130(1), 16-24. doi:10.1111/acps.12193

GIP / Zorginstituut Nederland (2016).

Goren, J. L., Rose, A. J., Engle, R. L., Smith, E. G., Christopher, M. L., Rickles, N. M., . . McCullough, M. B. (2016). Organizational Characteristics of Veterans Affairs Clinics With High and Low Utilization of Clozapine. Psychiatr Serv, 67(11), 1189-1196. doi:10.1176/appi.ps.201500506

Hayes, R. D., Downs, J., Chang, C. K., Jackson, R. G., Shetty, H., Broadbent, M., . . Stewart, R. (2015). The effect of clozapine on premature mortality: an assessment of clinical monitoring and other potential confounders. Schizophr Bull, 41(3), 644-655. doi:10.1093/schbul/sbu120

Howes, O. D., Vergunst, F., Gee, S., McGuire, P., Kapur, S., \& Taylor, D. (2012). Adherence to treatment 
guidelines in clinical practice: study of antipsychotic treatment prior to clozapine initiation. $\mathrm{Br} J$ Psychiatry, 201(6), 481-485. doi:10.1192/bjp.bp.111.105833

Ingimarsson, O., MacCabe, J. H., Haraldsson, M., Jonsdottir, H., \& Sigurdsson, E. (2016). Neutropenia and agranulocytosis during treatment of schizophrenia with clozapine versus other antipsychotics: an observational study in Iceland. BMC Psychiatry, 16(1), 441. doi:10.1186/s12888-016-1167-0

Kane, J. M. (2017). Clozapine Reduces All-Cause Mortality. Am J Psychiatry, 174(10), 920-921. doi:10.1176/appi.ajp.2017.17070770

Kelly, D. L., McMahon, R. P., Liu, F., Love, R. C., Wehring, H. J., Shim, J. C., . . Conley, R. R. (2010). Cardiovascular disease mortality in patients with chronic schizophrenia treated with clozapine: a retrospective cohort study. J Clin Psychiatry, 71(3), 304-311. doi:10.4088/JCP.08m04718yel

Kuo, C. J., Tsai, S. Y., Lo, C. H., Wang, Y. P., \& Chen, C. C. (2005). Risk factors for completed suicide in schizophrenia. J Clin Psychiatry, 66(5), 579-585.

Legge, S. E., Hamshere, M., Hayes, R. D., Downs, J., O’Donovan, M. C., Owen, M. J., . . MacCabe, J. H. (2016). Reasons for discontinuing clozapine: A cohort study of patients commencing treatment. Schizophr Res, 174(1-3), 113-119. doi:10.1016/j.schres.2016.05.002

Machielsen, M., Beduin, A. S., Dekker, N., Kahn, R. S., Linszen, D. H., van Os, J., . . Myin-Germeys, I. (2012). Differences in craving for cannabis between schizophrenia patients using risperidone, olanzapine or clozapine. J Psychopharmacol, 26(1), 189-195. doi:10.1177/0269881111408957

Malalagama, G., Bastiampillai, T., \& Dhillon, R. (2011). Clozapine use in Australia. Australas Psychiatry, 19(2), 175. doi:10.3109/10398562.2010.525646

McEvoy, J. P., Freudenreich, O., \& Wilson, W. H. (1999). Smoking and therapeutic response to clozapine in patients with schizophrenia. Biol Psychiatry, 46(1), 125-129.

Melle, I., Olav Johannesen, J., Haahr, U. H., Ten Velden Hegelstad, W., Joa, I., Langeveld, J., . . Friis, S. (2017). Causes and predictors of premature death in first-episode schizophrenia spectrum disorders. World Psychiatry, 16(2), 217-218. doi:10.1002/wps.20431

Moncrieff, J. (2006). Does antipsychotic withdrawal provoke psychosis? Review of the literature on rapid onset psychosis (supersensitivity psychosis) and withdrawal-related relapse. Acta Psychiatr Scand, 114(1), 3-13. doi:10.1111/j.1600-0447.2006.00787.x

Nielsen, J., Roge, R., Schjerning, O., Sorensen, H. J., \& Taylor, D. (2012). Geographical and temporal variations in clozapine prescription for schizophrenia. Eur Neuropsychopharmacol, 22(11), 818-824. doi:10.1016/j.euroneuro.2012.03.003

Nielsen, J., Thode, D., Stenager, E., Andersen, K. O., Sondrup, U., Hansen, T. N., . . le Quach, P. (2012). Hematological clozapine monitoring with a point-of-care device: a randomized cross-over trial. Eur Neuropsychopharmacol, 22(6), 401-405. doi:10.1016/j.euroneuro.2011.10.001

Patchan, K. M., Richardson, C., Vyas, G., \& Kelly, D. L. (2015). The risk of suicide after clozapine discontinuation: Cause for concern. Ann Clin Psychiatry, 27(4), 253-256.

Patel, M. X., Bishara, D., Jayakumar, S., Zalewska, K., Shiers, D., Crawford, M. J., \& Cooper, S. J. (2014). Quality of prescribing for schizophrenia: evidence from a national audit in England and Wales. Eur Neuropsychopharmacol, 24(4), 499-509. doi:10.1016/j.euroneuro.2014.01.014

Ringback Weitoft, G., Berglund, M., Lindstrom, E. A., Nilsson, M., Salmi, P., \& Rosen, M. (2014). 
Mortality, attempted suicide, re-hospitalisation and prescription refill for clozapine and other antipsychotics in Sweden-a register-based study. Pharmacoepidemiol Drug Saf, 23(3), 290-298. doi: $10.1002 /$ pds.3567

Rosenheck, R., Leslie, D., Sint, K., Lin, H., Robinson, D. G., Schooler, N. R., . . Kane, J. M. (2016). Cost-Effectiveness of Comprehensive, Integrated Care for First Episode Psychosis in the NIMH RAISE Early Treatment Program. Schizophr Bull, 42(4), 896-906. doi:10.1093/schbul/sbv224

Saha, S., Chant, D., Welham, J., \& McGrath, J. (2005). A systematic review of the prevalence of schizophrenia. PLoS Med, 2(5), e141. doi:10.1371/journal.pmed.0020141

Sin, J., \& Gamble, C. (2003). Managing side-effects to the optimum: valuing a client's experience. J Psychiatr Ment Health Nurs, 10(2), 147-153.

Stroup, T. S., Gerhard, T., Crystal, S., Huang, C., \& Olfson, M. (2014). Geographic and clinical variation in clozapine use in the United States. Psychiatr Serv, 65(2), 186-192. doi:10.1176/appi.ps.201300180

Stroup, T. S., Gerhard, T., Crystal, S., Huang, C., \& Olfson, M. (2016). Comparative Effectiveness of Clozapine and Standard Antipsychotic Treatment in Adults With Schizophrenia. Am J Psychiatry, 173(2), 166-173. doi:10.1176/appi.ajp.2015.15030332

Taipale, H., Mittendorfer-Rutz, E., Alexanderson, K., Majak, M., Mehtala, J., Hoti, F., . . Tiihonen, J. (2017). Antipsychotics and mortality in a nationwide cohort of 29,823 patients with schizophrenia. Schizophr Res. doi:10.1016/j.schres.2017.12.010

Taipale, H., Tanskanen, A., Mehtala, J., Vattulainen, P., Correll, C. U., \& Tiihonen, J. (2020). 20-year follow-up study of physical morbidity and mortality in relationship to antipsychotic treatment in a nationwide cohort of 62,250 patients with schizophrenia (FIN20). World Psychiatry, 19(1), 61-68. doi:10.1002/wps.20699

Termorshuizen, F., Wierdsma, A. I., Smeets, H. M., Visser, E., Drukker, M., Nijman, H., \& Sytema, S. (2013). Cause-specific mortality among patients with psychosis: disentangling the effects of age and illness duration. Psychosomatics, 54(6), 536-545. doi:10.1016/j.psym.2013.05.011

Tiihonen, J., Lonnqvist, J., Wahlbeck, K., Klaukka, T., Niskanen, L., Tanskanen, A., \& Haukka, J. (2009). 11-year follow-up of mortality in patients with schizophrenia: a population-based cohort study (FIN11 study). Lancet, 374(9690), 620-627. doi:10.1016/s0140-6736(09)60742-x

Tungaraza, T. E., \& Farooq, S. (2015). Clozapine prescribing in the UK: views and experience of consultant psychiatrists. Ther Adv Psychopharmacol, 5(2), 88-96. doi:10.1177/2045125314566808

Ucok, A., Cikrikcili, U., Karabulut, S., Salaj, A., Ozturk, M., Tabak, O., \& Durak, R. (2015). Delayed initiation of clozapine may be related to poor response in treatment-resistant schizophrenia. Int Clin Psychopharmacol, 30(5), 290-295. doi:10.1097/yic.0000000000000086

Valdes-Stauber, J., Putzhammer, A., \& Kilian, R. (2014). [Decentralized outpatient teams in communitybased psychiatric care: comparison of two Bavarian rural catchment areas]. Nervenarzt, 85(5), 596605. doi:10.1007/s00115-013-3836-2

Vanasse, A., Blais, L., Courteau, J., Cohen, A. A., Roberge, P., Larouche, A., . . Delorme, A. (2016). Comparative effectiveness and safety of antipsychotic drugs in schizophrenia treatment: a realworld observational study. Acta Psychiatr Scand, 134(5), 374-384. doi:10.1111/acps.12621

Verdoux, H., Pambrun, E., Cortaredona, S., Coldefy, M., Le Neindre, C., Tournier, M., \& Verger, P. 
(2016). Geographical disparities in prescription practices of lithium and clozapine: a communitybased study. Acta Psychiatr Scand, 133(6), 470-480. doi:10.1111/acps.12554

Verdoux, H., Quiles, C., Bachmann, C. J., \& Siskind, D. (2018). Prescriber and institutional barriers and facilitators of clozapine use: A systematic review. Schizophr Res, 201, 10-19. doi:10.1016/j. schres.2018.05.046

Vermeulen, J. M., van Rooijen, G., van de Kerkhof, M. P. J., Sutterland, A. L., Correll, C. U., \& de Haan, L. (2019). Clozapine and Long-Term Mortality Risk in Patients With Schizophrenia: A Systematic Review and Meta-analysis of Studies Lasting 1.1-12.5 Years. Schizophr Bull, 45(2), 315329. doi:10.1093/schbul/sby052

Walker, A. M., Lanza, L. L., Arellano, F., \& Rothman, K. J. (1997). Mortality in current and former users of clozapine. Epidemiology, 8(6), 671-677.

Wheeler, A. J. (2008). Treatment pathway and patterns of clozapine prescribing for schizophrenia in New Zealand. Ann Pharmacother, 42(6), 852-860. doi:10.1345/aph.1K662

Wimberley, T., MacCabe, J. H., Laursen, T. M., Sorensen, H. J., Astrup, A., Horsdal, H. T., . . Stovring, H. (2017). Mortality and Self-Harm in Association With Clozapine in Treatment-Resistant Schizophrenia. Am J Psychiatry, 174(10), 990-998. doi:10.1176/appi.ajp.2017.16091097 



\section{Nederlandse samenvatting}

\section{Inleiding}

Dit proefschrift gaat over verschillende aspecten van het gebruik van het antipsychoticum clozapine. De aanleiding voor het schrijven van dit proefschrift was dat dit middel te weinig wordt voorgeschreven en dat het vaak lang duurt voordat het wordt voorgeschreven. Dit is wereldwijd een probleem en er is nauwelijks onderzoek naar interventies om de prescriptiecijfers te verhogen of om de vertraging voordat het wordt voorgeschreven te verkleinen. Het eerste deel van dit proefschrift gaat over de prescriptiecijfers in Nederland en het stimuleren van clozapine-gebruik. Wij hebben ons daarbij gericht op de verplichte en tijdrovende monitoring die nodig is bij het starten van clozapine en die een belemmering kan zijn voor voorschrijvers. Daarnaast is er onduidelijkheid over de relatie tussen clozapine-gebruik en sterfte, mogelijk is dit ook een belemmering bij het voorschrijven. In het tweede deel van dit proefschrift onderzoeken we daarom de relatie tussen sterfte en clozapine. $\mathrm{Na}$ een algemene uitleg over psychoses, antipsychotica en met name clozapine, volgt een korte samenvatting van de onderzoeken die in het kader van dit proefschrift gedaan zijn. We sluiten deze samenvatting af met de conclusie.

\section{Psychose/schizofrenie}

Bij een psychose is er sprake van wanen en/of hallucinaties. Psychoses kunnen voorkomen bij verschillende psychiatrische stoornissen, maar met name schizofrenie wordt gekenmerkt door wanen en/of hallucinaties, deze worden dan positieve symptomen genoemd. Schizofrenie komt voor bij ongeveer 0,5 tot $1 \%$ van de totale bevolking. De behandeling van psychoses is voor een belangrijk deel medicamenteus door middel van antipsychotica, aangevuld met psychologische behandeling. Bij ongeveer een derde van de patiënten is de behandeling met antipsychotica onvoldoende effectief, deze patiënten worden therapieresistent genoemd. Clozapine is het enige antipsychoticum dat bewezen effectief is bij therapieresistente psychoses. Het zou volgens de richtlijnen voorgeschreven moeten worden als positieve symptomen aanwezig blijven ondanks dat er 2 andere middelen geprobeerd zijn. Daarnaast kan het bij patiënten met een psychotische stoornis ook voorgeschreven worden als er ondanks het gebruik van antipsychotica sprake blijft van agressie, suïcidaliteit, onbehandelbare extrapiramidale stoornissen of aanhoudend middelenmisbruik. Dat dit medicijn niet als eerste of tweede keus gebruikt wordt, komt omdat clozapine een aantal potentieel gevaarlijke bijwerkingen heeft. De bekendste daarvan is agranulocytose, een afname van het aantal witte bloedcellen die levensbedreigend is. Hierdoor is intensieve controle van de witte bloedcellen (vooral in het begin) noodzakelijk en verplicht. In Nederland moet deze controle wekelijks plaatsvinden in de eerste 18 weken van het gebruik en daarna maandelijks zo lang de patiënt clozapine gebruikt. Andere zeldzame, maar ook gevaarlijke bijwerkingen zijn myocarditis, ileus en longembolie. De kans op metabole bijwerkingen is bij het 
gebruik van dit middel ook groter dan bij het gebruik van andere antipsychotica (op olanzapine na). Alle genoemde bijwerkingen en de intensieve monitoring die nodig is om complicaties te voorkomen kunnen van invloed zijn op het voorschrijven van clozapine en er (mede) de oorzaak van zijn dat het wereldwijd te weinig of pas laat wordt voorgeschreven. Artsen/psychiaters zijn verantwoordelijk voor de intensieve monitoring om tijdig te herkennen dat bepaalde bijwerkingen optreden. Dit kost niet alleen veel tijd, maar kan ook voelen als een grote verantwoordelijkheid. Daarnaast hebben artsen/ psychiaters het idee dat door de regelmatige bloedcontroles veel patiënten dit middel niet willen gebruiken. De mogelijk gevaarlijke bijwerkingen in combinatie met de noodzakelijke intensieve controle kunnen een reden zijn dat clozapine wereldwijd veel te weinig wordt voorgeschreven.

Het eerste deel van dit proefschrift gaat over prescriptiecijfers van clozapine en het stimuleren van clozapine-gebruik. Hoofdstuk 2 gaat over een onderzoek naar prescriptie en onder-prescriptie van clozapine, dat voorafging aan de interventiestudie beschreven in hoofdstuk 3. We bepaalden welk percentage van de patiënten met een psychotische stoornis al clozapine gebruikt. Daarnaast ontwikkelden we een beslisboom en onderzochten we welk percentage van deze patiënten een indicatie voor dit middel had, maar het niet gebruikte. Aan het onderzoek deden 20 ambulante FACT-teams (Flexible Assertive Community Treatment) en 3 VIP-teams (Vroege Interventie Psychose) van 4 verschillende psychiatrische instellingen mee. $\mathrm{Na}$ het beoordelen van 2.286 patiënten met een psychotische stoornis vonden we dat gemiddeld 23,0\% clozapine gebruikte. De verschillen tussen de teams waren groot en statistisch significant, de range was van 8,8 tot $34,7 \%$ per team. Het percentage patiënten dat wel een indicatie had voor clozapine maar het middel niet kreeg, was gemiddeld 6,8\%. Ook hier waren de verschillen tussen de teams groot: (van 1,7 tot 15,6\% per team). In het algemeen was het aantal patiënten dat al clozapine gebruikte groter dan verwacht en groter dan in veel andere landen gevonden is.

In hoofdstuk 3 beschrijven we het onderzoek naar het effect van een interventie om de prescriptiecijfers van clozapine te verbeteren. In dit cluster-gerandomiseerde onderzoek, testten we de hypotheses dat psychiaters clozapine vaker zouden voorschrijven als ze de intensieve monitoring zouden kunnen delegeren aan een verpleegkundig specialist (VS), dat de monitoring door een VS minstens zo veilig is als die door een psychiater en dat bij monitoring door een VS minder patiënten vroegtijdig zouden stoppen met dit middel. De psychiaters, VS'en en patiënten waren blind voor de eerste hypothese, zij wisten dus niet dat we vooral wilden onderzoeken of de prescriptie stijgt als de monitoring uitbesteed kan worden aan een VS. Alleen de tweede vraagstelling naar de veiligheid van de monitoring door de VS was hun bekend. Om te kunnen testen of psychiaters meer patiënten op clozapine zouden instellen als zij de monitoring konden delegeren 
aan de VS (de interventieconditie), was het nodig dat alle patiënten behorende bij één psychiater/team zich in dezelfde conditie bevonden. Omdat sommige VS'en voor meerdere teams werkten hebben we besloten de VS'en te randomiseren. Hierdoor werd voorkomen dat een VS bij een patiënt uit het ene team verantwoordelijk was voor de monitoring en zich bij een patiënt uit een ander team volledig afzijdig moet houden. $\mathrm{Na}$ de randomisatie werden de VS'en, die in de interventieconditie geloot waren, getraind om de clozapine-monitoring goed uit te kunnen voeren. Zoals we hadden verwacht, schreven psychiaters ongeveer twee keer zo vaak clozapine voor als er een VS beschikbaar was voor de monitoring. Echter, het aantal patiënten dat startte was heel klein en het verschil tussen de condities was niet significant. Slechts 11 van de $173(6,4 \%)$ patiënten met een indicatie aan het begin van de onderzoeksperiode is tijdens de inclusieperiode met clozapine gestart. Hierdoor was er onvoldoende power om een significant resultaat te verkrijgen. Er zijn tijdens de onderzoeksperiode meer patiënten gestart dan de hiervoor genoemde 11. Deze patiënten hadden geen indicatie voor clozapine aan het begin van de onderzoeksperiode, maar zij ontwikkelden later pas een indicatie (bijvoorbeeld door toename van de symptomen). Bij deze patiënten zagen we hetzelfde resultaat, in de interventieconditie startten ongeveer 2 keer zoveel patiënten als in de controleconditie. Dit resultaat was, mogelijk door de kleine aantallen, net zomin significant. We vonden geen verschillen in veiligheid tussen de monitoring door de psychiater en die door de VS. Gemiddeld werd 71,2\% van de labcontroles uitgevoerd, met grote verschillen tussen de teams. Ook in voortijdig stoppen met clozapine waren er geen significante verschillen tussen de 2 condities.

\section{Clozapine en sterfte}

Het tweede deel van dit proefschrift gaat over de relatie tussen clozapine en sterfte. In het algemeen is de levensverwachting van mensen met een ernstige psychiatrische aandoening 10 tot 20 jaar korter in vergelijking met de rest van de bevolking. Door de metabole bijwerkingen zou langdurig gebruik van clozapine een negatief effect kunnen hebben op cardiovasculaire sterfte in vergelijking tot langdurig gebruik van andere antipsychotica. Aan de andere kant is in eerdere onderzoeken gevonden dat het risico op suïcide tijdens het gebruik van clozapine verlaagd zou zijn. Een aantal onderzoeken liet zelfs een (sterk) verlaagde totale sterfte tijdens het gebruik van clozapine zien in vergelijking met het gebruik van andere antipsychotica, maar niet in alle onderzoeken werd dit verschil gevonden. Hoofdstuk 4 begint daarom met een commentaar op onderzoeken naar clozapine en mortaliteit. Hierin bespreken we de bias en de methodologische fouten die mogelijk van invloed waren op de resultaten van studies die een sterk verlaagde sterfte vonden tijdens het gebruik van clozapine. Een voorbeeld van bias is 'survivor bias'. Dit speelt een rol in onderzoeken naar clozapine en suïcide omdat clozapine vaak pas jaren na het ontstaan van de psychotische stoornis wordt voorgeschreven, terwijl suïcide vooral voorkomt in de eerste jaren. Hier moet voor gecorrigeerd worden. Een voorbeeld 
van een methodologische fout is dat er in een meta-analyse over clozapine en sterfte niet gecorrigeerd werd voor de jongere leeftijd van gebruikers van clozapine.

In Hoofdstuk 5 hebben we 2 andere bronnen van bias bestudeerd die mogelijk de resultaten van onderzoeken naar clozapine en sterfte kunnen beïnvloeden. De eerste vorm van bias is dat clozapine mogelijk vaker wordt voorgeschreven aan relatief gezonde patiënten, waardoor de sterfte onder deze patiënten wel lager is, maar niet als gevolg van clozapinegebruik. De tweede vorm is dat de intensieve monitoring die nodig is tijdens clozapinegebruik ervoor kan zorgen dat lichamelijke problemen eerder herkend en sneller behandeld worden. Doordat in het algemeen lichamelijke problemen bij patiënten met ernstige psychiatrische aandoeningen te weinig herkend en behandeld worden, zou er een verschil kunnen zijn tussen gebruikers van clozapine en andere antipsychotica. We hebben onderzoek gedaan naar deze vormen van bias met behulp van databestanden van een verzekeringsmaatschappij. We toonden aan dat de patiënten die begonnen met clozapine minstens evenveel cardiovasculaire of diabetische aandoeningen hadden als de patiënten die begonnen met andere antipsychotica. Het lijkt er dus op dat zij niet gezonder waren bij de start van het gebruik en dat het niet waarschijnlijk is dat de verlaagde sterfte daardoor verklaard wordt. Voor het onderzoek naar de tweede vorm van bias hebben we het gebruik van clozapine alleen vergeleken met het gebruik van olanzapine, omdat alleen olanzapine een vergelijkbaar bijwerkingenprofiel en daarmee samenhangend risico op metabool syndroom heeft. We vonden dat er tijdens het gebruik van clozapine vaker en in een vroeger stadium nieuwe cardiovasculaire medicatie of antidiabetica werden voorgeschreven dan tijdens het gebruik van olanzapine. Dit bevestigt onze hypothese dat de intensieve monitoring die nodig is bij het instellen op/gebruik van clozapine bij kan dragen aan een betere behandeling/preventie van cardiovasculaire problemen en diabetes.

In hoofdstuk 6 hebben we de relatie tussen clozapine en sterfte onderzocht met behulp van Deense nationale registers. Hierbij hebben we niet alleen gekeken naar het antipsychoticum gebruikt ten tijde van overlijden ("tijdens het gebruik" of "huidig gebruik"), maar ook naar het antipsychoticum dat op dat tijdstip het langst gebruikt was ("cumulatief gebruik"). We hebben de analyses in een incidentiecohort uitgevoerd (patiënten die voor het eerst een diagnose psychotische stoornis kregen tijdens de observatietijd van 19,5 jaar), maar ook in een prevalentiecohort (alle patiënten met een diagnose psychotische stoornis, dus ook degenen die al een diagnose hadden voor de observatietijd begon). We hebben gekeken naar totale sterfte, sterfte door suïcide en cardiovasculaire sterfte en hebben een analyse met en één zonder correctie voor cardiovasculaire/diabetes diagnoses en de behandeling daarvan gedaan. We hebben ervoor gekozen om dit onderzoek in een Scandinavisch land uit te voeren omdat daar de mogelijkheid bestaat om nationale registers aan elkaar te koppelen. Hierdoor is van alle 
inwoners bekend welke medicijnen zij gebruiken, voor welke diagnoses zij behandeld werden en wat de doodsoorzaken zijn. $\mathrm{Na}$ het selecteren van alle patiënten met een psychotische stoornis waren er in totaal 50881 patiënten in het prevalentiecohort en 22 110 in het incidentiecohort. We hebben onderzocht of gebruik van clozapine geassocieerd was met een hogere of lagere sterfte dan het gebruik van andere antipsychotica. We vonden geen aanwijzingen voor een lagere totale of cardiovasculaire sterfte tijdens clozapinegebruik, noch in het prevalentiecohort, noch in het incidentiecohort. Bij sterfte door suïcide zagen we wel een lagere sterfte tijdens het gebruik van clozapine vergeleken met het gebruik van andere antipsychotica, maar dat was niet significant. Opvallend waren de tegengestelde resultaten voor huidig en cumulatief gebruik van clozapine en suïcide. Tijdens clozapine-gebruik waren er nauwelijks suïcides, maar in de categorie cumulatief gebruik, 0 tot 1 jaar was er een verhoogd risico op suïcide in de clozapine-groep. (Cumulatief gebruik van 0-1 jaar betekent dat de patiënt clozapine langer heeft gebruikt dan enig ander antipsychoticum, maar niet langer dan 1 jaar.) Aangezien de sterfte tijdens het gebruik verlaagd was, zullen de patiënten uit deze laatste groep op een enkeling na gestopt zijn met het gebruik vóór het moment van de suïcide. Een verklaring voor een verhoogd suïcide-risico na het stoppen met clozapine is dat de psychotische symptomen (die suïcidaliteit kunnen veroorzaken) weer toenemen, bij abrupt stoppen zelfs in heviger mate dan voor het starten met clozapine. Een andere verklaring voor een verhoogd suïcide-risico na het stoppen met clozapine is wanhoop. De patiënt heeft immers vaak begrepen dat clozapine een laatste redmiddel is en dat de prognose niet beter wordt als dit middel gestaakt moet worden wegens bijwerkingen of gebrek aan effect. Het was geruststellend om te zien dat cumulatief clozapine-gebruik van 6 jaar of langer, ondanks de metabole bijwerkingen van dit middel, niet gepaard ging met een hogere totale of cardiovasculaire sterfte in vergelijking met het gebruik van andere antipsychotica.

\section{Conclusie}

De belangrijkste doelen in dit proefschrift waren het evalueren van een interventie om het gebruik van clozapine te stimuleren en het verduidelijken van de relatie tussen clozapine en mortaliteit. We vonden dat de prescriptiecijfers in Nederland hoog waren, maar concludeerden ook dat de inzet van de VS bij de monitoring niet afdoende is om het probleem van onderbehandeling met clozapine op te lossen. De verschillen tussen de condities waren klein, terwijl de verschillen tussen de teams groot blijven. Het vooraf identificeren van patiënten met een indicatie voor clozapine heeft niet geleid tot een duidelijke toename van het voorschrijven van clozapine.

We hebben geen aanwijzingen gevonden dat clozapine wordt voorgeschreven aan relatief gezonde patiënten. Patiënten die clozapine gebruiken krijgen mogelijk wel een meer adequate somatische behandeling in vergelijking met patiënten die andere antipsychotica 
gebruiken. In de Deense studie zagen we niet dat dit de relatie tussen clozapine en sterfte duidelijk beïnvloedt. We hebben laten zien dat onderzoek naar de relatie tussen clozapine en sterfte ingewikkeld is en dat de manier van analyseren de resultaten beïnvloedt. In ons commentaar op andere onderzoeken laten we zien dat vertekening en methodologische fouten in diverse studies geleid hebben tot een te rooskleurige kijk op het verband tussen clozapine en mortaliteit. Wij vonden in ons onderzoek in Denemarken geen grote verschillen in sterfte tussen huidig of cumulatief gebruik van clozapine en gebruik van andere antipsychotica. Belangrijk is dat zelfs langdurig gebruik van dit middel de cardiovasculaire sterfte niet lijkt te verhogen. De resultaten wijzen wel in de richting van een beschermend effect van clozapine tegen suïcide tijdens het gebruik. Onze resultaten suggereren echter ook een verhoogd risico op suïcide voor degenen die het gebruik van clozapine stopten, met name na gebruik korter dan een jaar. Nauwgezette monitoring van suïcidaliteit lijkt daarom een belangrijk aandachtspunt te zijn na beëindiging van clozapine. 


\section{Impactparagraaf}

In dit proefschrift stonden twee doelen centraal. Het eerste doel was het evalueren van een interventie gericht op het bevorderen van het voorschrijven van het antipsychotische middel clozapine door de behandelend psychiater. Het tweede doel was meer duidelijkheid verkrijgen over de relatie tussen clozapine en sterfte.

Clozapine is het enige antipsychotische medicijn dat bewezen effectief is bij patiënten met een therapieresistente psychose. Zowel volgens nationale als internationale richtlijnen zou het voorgeschreven moeten worden als twee eerdere middelen onvoldoende effectief waren. Het gebruik van clozapine is van groot belang omdat ongeveer de helft van de patiënten daarmee alsnog psychosevrij wordt en het risico op suïcide afneemt. Het spreekt voor zich dat dit ook belangrijk is voor de familieleden van patiënten en voor de behandelaren. Helaas zijn de prescriptiecijfers internationaal lager dan verwacht zou worden. Een oorzaak hiervan is de verplichte intensieve monitoring tijdens de eerste weken van het gebruik van dit middel om een potentieel gevaarlijke bijwerking (agranulocytose) op tijd te signaleren. In Nederland zijn in de eerste 18 weken van het gebruik wekelijkse bloedcontroles verplicht. Dat vormt voor de behandelend psychiater een grote belasting. Hij of zij moet erop toezien dat de patiënt naar het laboratorium gaat en dat de uitslag hem of haar op tijd bereikt. Een andere mogelijke oorzaak is bezorgdheid van de psychiater over gewichtstoename bij de patiënt en het ontstaan van een metabool syndroom.

Onze interventie bestond hierin dat we de psychiater de mogelijkheid boden om de clozapine-monitoring aan verpleegkundig specialisten te delegeren. Deze specialisten werden voorafgaand aan het onderzoek getraind om deze taak goed te kunnen uitvoeren. Vóór aanvang van dit onderzoek werd vastgesteld hoeveel patiënten al clozapine gebruikten en welke patiënten wel een indicatie hadden, maar het nog niet gebruikten. We vonden dat het percentage patiënten in de 23 deelnemende ambulante teams dat al clozapine gebruikte relatief hoog was. Daarnaast vonden we dat noch de mogelijkheid tot delegeren van de monitoring aan de verpleegkundig specialist, noch het vooraf aanwijzen van patiënten met een indicatie, tot een substantiële toename van de prescriptiecijfers leidde. We vonden weliswaar dat er in de interventiegroep ongeveer twee keer zoveel patiënten werden ingesteld op clozapine als in de controlegroep (8,5\% vs.4,4\%), maar het verschil in absolute aantallen was klein.

Desalniettemin leverde het onderzoek twee belangrijke positieve resultaten op. In de eerste plaats bleek dat de clozapine-monitoring door de verpleegkundig specialist minstens net zo veilig werd uitgevoerd als door de psychiater: het percentage van de verplichte bloedcontroles dat werd uitgevoerd in de interventieconditie (door de 
verpleegkundig specialist, 71\%) was net iets hoger dan in de controleconditie (door de psychiater, 67\%). Deze uitkomst is ook van belang voor beleidsmakers. Wij verwachten daarom dat verpleegkundig specialisten in de komende jaren vaker clozapine gaan voorschrijven en de monitoring vaker zullen uitvoeren. Ons onderzoek draagt bij aan de onderbouwing van deze ontwikkeling.

Een tweede positief resultaat van de inschakeling van een verpleegkundig specialist is het succesvol instellen in een ambulante setting, buiten het psychiatrisch ziekenhuis. Sommige ambulant werkende psychiaters waren vóór het onderzoek van mening dat de instelling in de eerste weken zoveel toezicht vereist dat het op een klinische opnameafdeling moet, maar het werd hen duidelijk dat dit met de hulp van een verpleegkundig specialist meestal niet meer nodig is. Als de psychiater een opname niet langer noodzakelijk acht, zal de drempel voor patiënten om met dit middel te beginnen ook lager worden.

Ambulant instellen is daarnaast ook kostenbesparend. Voor beleidsmakers is het ook van belang om te weten dat er andere maatregelen moeten worden ingezet om het voorschrijven van clozapine substantieel te bevorderen. Het screenen op indicaties alleen is onvoldoende, daarna zouden eisen gesteld kunnen worden aan het voorschrijfbeleid. Men kan hier denken aan het opnemen van deze eisen in de audits van FACTteams (ambulante teams voor de ambulante behandeling van patiënten met ernstige psychiatrische aandoeningen) of het verbinden van financiële consequenties aan het al dan niet voorschrijven van clozapine volgens de richtlijnen.

Doelgroepen van ons onderzoek zijn patiënten, familieleden, behandelaren (vooral psychiaters en verpleegkundig specialisten) en, last but not least, beleidsmakers. We hebben de voornaamste resultaten daarom gepubliceerd in het tijdschrift "Administration and policy in mental health and mental health services research". Daarnaast hebben we er bekendheid aan gegeven op nationale en internationale congressen voor psychiaters en in het Nederlandstalig tijdschrift Psyfar VS (een psychofarmacologisch tijdschrift voor de verpleegkundig specialist). Voor patiënten en familieleden is informatie over ons onderzoek op de site psychosenet.nl geplaatst.

Het tweede doel van dit proefschrift was meer duidelijkheid krijgen over de relatie tussen clozapine en sterfte. Clozapine vergroot de kans op het metabool syndroom en zou daarmee de levensverwachting kunnen verkorten. Desondanks liet een aantal eerdere onderzoeken een sterk verlaagde sterfte in relatie tot clozapine-gebruik zien. Wij hebben beschreven dat deze onderzoeken de relatie tussen clozapine en sterfterisico te rooskleurig voorstellen omdat met een aantal belangrijke bronnen van vertekening geen rekening werd gehouden. Een voorbeeld hiervan is het buiten beschouwing laten van leeftijd, terwijl patiënten die clozapine gebruiken over het algemeen jonger zijn dan 
patiënten die andere middelen gebruiken. Daarnaast wordt clozapine (omdat het een derde stap is in de behandeling) minder vaak voorgeschreven in de eerste jaren na een eerste psychose, terwijl juist in die jaren het suïciderisico het grootst is.

In een onderzoek met Nederlandse data onderzochten we of patiënten die starten met clozapine ook gezonder zijn. Het is immers denkbaar dat psychiaters uit bezorgdheid over bijwerkingen dit middel eerder aan gezonde mensen voorschrijven en dat dit voorschrijfgedrag de lagere sterfte verklaart. Wij onderzochten ook of zij door de intensieve monitoring betere (preventieve) somatische zorg kregen. We concludeerden dat er bij het starten geen verschil was in lichamelijke gezondheid, maar dat gedurende het gebruik van clozapine wel meer medicatie wordt voorgeschreven om bijvoorbeeld het metabool syndroom te voorkomen, vergeleken met patiënten die een ander middel kregen met net zo veel metabole bijwerkingen (olanzapine).

In ons onderzoek met Deense data naar de relatie tussen clozapine en sterfte hebben we rekening gehouden met dit effect van betere preventieve somatische zorg. We vonden dat er nauwelijks verschillen zijn tussen gebruikers van clozapine en gebruikers van andere antipsychotica waar het gaat om het risico op algehele en cardiovasculaire sterfte. Het risico op suïcide was daarentegen tijdens clozapinegebruik juist wel lager. Deze resultaten kunnen bij psychiaters de terughoudendheid verminderen om dit middel voor te schrijven. Voor patiënten en familieleden kan het een geruststelling zijn dat dit middel hun levensverwachting niet verkort in vergelijking met andere antipsychotica.

De relatie die wij vonden tussen clozapine en suïcide was opmerkelijk. Tijdens het gebruik was het risico op suïcide duidelijk verlaagd. Een voorgeschiedenis van gebruik van clozapine gedurende $0-1$ jaar bleek echter juist geassocieerd met een verhoogde kans op suïcide. Omdat suïcidaliteit de reden geweest kan zijn om met clozapine te starten, suïcidaliteit is immers ook een indicatie voor clozapine, kan een toename van suïcidaliteit na het stoppen een logisch gevolg zijn. Dit betekent dat behandelaren bij patiënten die binnen een jaar met het middel stoppen extra alert moeten zijn op de mogelijkheid van een suïcidepoging.

Wij hopen met onze kritische evaluatie van eerdere onderzoeken en ons eigen onderzoek naar clozapine en sterfte in Denemarken een meer realistisch beeld gecreëerd te hebben dan dat geschetst door eerdere onderzoekers. Wij hebben deze resultaten gepubliceerd in wetenschappelijke tijdschriften (Schizophrenia Research, International Clinical Psychopharmacology, Acta Psychiatrica Scandinavica) en er ook bekendheid aan gegeven op verschillende congressen in binnen- en buitenland. 
Concluderend hopen wij met de in dit proefschrift beschreven onderzoeken enkele bezwaren tegen het gebruik van clozapine ondervangen te hebben: zowel het praktische bezwaar van de intensieve monitoring door de psychiater (de verpleegkundig specialist kan dit net zo goed doen) als sommige bijwerkingen: we vinden weliswaar geen verlaagde sterfte, maar ook geen verhoogde sterfte. 


\section{List of publications}

Clozapine and mortality: A comparison with other antipsychotics in a nationwide Danish cohort study. van der Zalm Y, Foldager L, Termorshuizen F, Sommer IE, Nielsen J, Selten JP. Acta Psychiatr Scand. 2020 Dec 11. doi: 10.1111/acps.13267. Online ahead of print. PMID: 33306211

Delegating Clozapine Monitoring to Advanced Nurse Practitioners: An Exploratory, Randomized Study to Assess the Effect on Prescription and Its Safety. van der Zalm YC, Schulte PF, Bogers JPAM, Termorshuizen F, Marcelis M, van Piere MAGB, Sommer IE, Selten JP; CLOZ-NP Study Group.

Adm Policy Ment Health. 2020 Jul;47(4):632-640. doi: 10.1007/s10488-020-01031-4. PMID: 32189094

Use of cardiovascular and antidiabetic drugs before and after starting with clozapine versus other antipsychotic drugs: a Dutch database study. van der Zalm Y, Termorshuizen F, Sommer IE, Selten JP.

Int Clin Psychopharmacol. 2020 Jan;35(1):36-41. doi: 10.1097/ YIC.0000000000000292.

PMID: 31714320

Concerns about bias in studies on clozapine and mortality.

van der Zalm YC, Termorshuizen F, Selten JP.

Schizophr Res. 2019 Feb;204:425-426. doi: 10.1016/j.schres.2018.08.017. Epub 2018 Aug 18.

PMID: 30126814

Prescription and Underprescription of Clozapine in Dutch Ambulatory Care. van der Zalm YC, Termorshuizen F, Schulte PF, Bogers JP, Marcelis M, Sommer IE, Selten JP.

Front Psychiatry. 2018 Jun 11;9:231. doi: 10.3389/fpsyt.2018.00231. eCollection 2018.

PMID: 29942266

Experiences of Patients in Acute and Closed Psychiatric Wards: A Systematic Review. Nugteren W, van der Zalm Y, Hafsteinsdóttir TB, van der Venne C, Kool N, van Meijel B. Perspect Psychiatr Care. 2016 Oct;52(4):292-300. doi: 10.1111/ppc.12125. Epub 2015 Jun 1.

PMID: 26033512 
Psychiatric nursing care for adult survivors of child maltreatment: a systematic review of the literature.

van der Zalm YC, Nugteren WA, Hafsteinsdóttir TB, van der Venne CG, Kool N, van Meijel B.

Perspect Psychiatr Care. 2015 Jan;51(1):71-8. doi: 10.1111/ppc.12059. Epub 2014 Jan 10. PMID: 24405168 


\section{Dankwoord}

Mijn promotie-onderzoek naar het instellen op clozapine startte in 2015. De vacature voor dit onderzoek naar het instellen op clozapine kwam precies op het goede moment en had direct mijn interesse gewekt. Ik had vlak daarvoor mijn studie verplegingswetenschappen afgerond en had jarenlang gewerkt op een afdeling waar een groot deel van de patiënten op clozapine was of werd ingesteld. Ik ben vol enthousiasme aan het onderzoek begonnen en ik had dit natuurlijk niet kunnen doen zonder de hulp en steun van anderen. Velen waren tijdens dit proces belangrijk voor het faciliteren, inspireren en motiveren, waardoor ik leuke, uitdagende en leerzame jaren heb gehad. Hen zou ik graag willen bedanken.

Allereerst gaat mijn dank uit naar alle personen uit de verschillende instellingen die hebben meegedaan aan het onderzoek naar het instellen op clozapine. Zonder hen was dit onderzoek niet mogelijk geweest. Ik dank de patiënten voor hun bereidheid informatie uit hun EPD te delen en de psychiaters voor hun hulp bij het invullen van de dataformulieren en het beoordelen van de indicaties voor clozapine. De verpleegkundig specialisten ben ik nog meer dank verschuldigd. Zij hebben niet alleen geholpen bij de dataverzameling, maar ook tijd vrijgemaakt voor het dossieronderzoek van de hele caseload. Met veel geduld hebben zij geholpen om van iedere patiënt vast te stellen of er wel of geen indicatie voor clozapine was, een zeer tijdrovende klus.

Veel dank ook voor de leden van het promotieteam: Jean-Paul Selten, Iris Sommer, Raphael Schulte en Fabian Termorshuizen.

Jean-Paul, jij bent al die jaren een zeer betrokken en toegankelijke promotor geweest. Je was altijd beschikbaar voor overleg en zeer geïnteresseerd in hoe de onderzoeken vorderden. Je was altijd bereid mee te denken als er problemen waren en ik heb veel geleerd van je adviezen en je feedback. Je positieve instelling is ook een eigenschap die ik gewaardeerd heb en die als een rode draad door onze samenwerking liep. Ik werd daar weer aan herinnerd toen ik je vroeg hoeveel proefschriften jij zelf had laten drukken. Je antwoordde dat je er in je optimisme 300 had laten drukken maar dat dat veel te veel was. Het tekent wel hoe jij bent. Op de momenten dat alles tegen leek te zitten, was jouw optimisme een belangrijke stimulans om door te gaan.

Iris, wat fijn dat je zo enthousiast was over het onderwerp en dank voor al jouw tips en feedback. Je hebt enorm geholpen met het organiseren van de kick-off van het onderzoek voor alle deelnemende psychiaters en verpleegkundig specialisten en daarmee bijgedragen aan een goede start van het onderzoek. Het was ook fijn om mijn (Engelse) presentatie voor het congres in Florence te kunnen oefenen bij jouw onderzoeksgroep. 
Ik had geen enkele ervaring met presenteren in het Engels en heb zeer nuttige feedback gekregen van jou en jouw andere promovendi.

Raphael, jouw mening is gedurende het hele proces heel waardevol geweest voor mij. Ik was direct onder de indruk van jouw kennis in combinatie met je enthousiasme over clozapine. Vanaf onze eerste kennismaking tot het laatste deel van het proefschrift heb ik veel feedback van je gekregen. Zowel bij het opzetten van de studie, het maken van de beslisboom als later bij de artikelen reageerde je altijd snel en heel kritisch, maar op een positieve en opbouwende manier. Ook jouw bijdrage aan de kick-off van het onderzoek en de scholing van de deelnemers was waardevol. Je niet aflatende nieuwsgierigheid heb $\mathrm{ik}$ als zeer inspirerend ervaren.

Fabian, het was prettig om met je samen te werken! Je bent bij alle onderzoeken betrokken geweest en ik heb veel van je geleerd over de statistiek in de verschillende onderzoeken. Met name bij de database-studies was je hulp onmisbaar. Ik vond het fijn om samen met jou andere studies over clozapine en mortaliteit kritisch te kunnen bekijken, je was oprecht geïnteresseerd in dit onderwerp en altijd bereid om mee te kijken en te denken. Ik heb ook goede herinneringen aan onze reis naar Denemarken. De intensieve samenwerken met een statisticus in een Deense universiteit op het platteland was een bijzondere ervaring.

De co-auteurs van de verschillende studies wil ik graag bedanken voor jullie bijdrage aan de artikelen. Marieke van Piere, ook dank voor je hulp bij de scholing van de verpleegkundig specialisten. Jan Bogers, jij had ook een belangrijke rol bij de scholing van de verpleegkundig specialisten en bij de kick-off van het onderzoek. Voorafgaand aan het onderzoek had ik al lang met jou samengewerkt. Op deze afdeling, waar de werkwijze drastisch veranderde (verbeterde) door jouw komst, had ik veel geleerd over clozapine. Dat heeft zeker bijgedragen aan mijn keuze om dit onderzoek te willen uitvoeren. Machteld Marcelis, hartelijk dank voor je bemiddeling waardoor wij het onderzoek ook binnen GGz Eindhoven konden uitvoeren. Natuurlijk ook bedankt voor je feedback op de artikelen.

Leslie, thank you very much for your almost infinite patience. We have exchanged hundreds of emails en you have sent me a really large stack of output. It was complicated and time-consuming, but I enjoyed every minute of it. I also want to thank you for your hospitality when Fabian and I went to Denmark.

De leden van de leescommissie wil ik hartelijk danken. Professor Berno van Meijel en dr. Maarten Bak, bedankt voor het lezen en goedkeuren van het proefschrift en wat jammer dat jullie niet bij de promotie aanwezig kunnen zijn. Professor van Amelsvoort, professor 
van Harten en dr. Bond-Veerman, ik wil jullie niet alleen danken voor het beoordelen en goedkeuren van het proefschrift, maar ook voor bereidheid om te opponeren.

Berno, jou wil ik nog speciaal bedanken voor de grote rol die jij gespeeld hebt in mijn keuze voor een promotietraject. Tijdens mijn studie verplegingswetenschappen was jij bereid om mij te begeleiden en kon ik mijn afstudeeronderzoek binnen jouw onderzoeksgroep doen. Zowel dit onderzoek bij Cokky van der Venne als de wetenschapsstage bij Nienke van der Voort, hebben mij geholpen om zelf ook onderzoeker te willen worden. Jij was zelfs bereid om een bezoek te brengen aan de raad van bestuur van Rivierduinen in het kasteel in Oegstgeest om mij daarbij te helpen. De intervisiemomenten op de dagen van de kenniskring GGZ verpleegkunde en de lezingen hebben mij gestimuleerd om verder te gaan in het onderzoek en het vertrouwen gegeven dat ik dat zou kunnen. Jouw gedrevenheid voor (verpleegkundig) onderzoek wordt gedeeld door alle leden van de kenniskring, waardoor dat voor mij een leerzame en inspirerende omgeving was en is. Mijn intervisiegenoten zou ik ook graag willen bedanken, ook jullie feedback op mijn artikelen heeft mij geholpen om deze uiteindelijk te kunnen publiceren.

GGZ Rivierduinen dank ik voor het financieel mogelijk maken van dit project. Mijn directe collega's en medepromovendi zijn natuurlijk ook belangrijk voor me geweest de afgelopen jaren. Jonas, korte tijd was jij mijn enige collega. Het was fijn om jou als ervaren promovendus advies te kunnen vragen. Jori, je was een belangrijke steun voor me. De door jou geïntroduceerde lunch-wandelingen hebben we altijd in ere gehouden. Rik, wat ben jij een onderzoeker in hart en nieren. Ik heb goede herinneringen aan de discussies over onderzoek, maar zeker ook aan onze jammerlijke pogingen om de puzzels van de AIVD te kraken. Carmen, ook jouw gezelschap heb ik erg op prijs gesteld. Ik vond het erg jammer dat ons wekelijkse contact en de lunchwandelingen ophielden door de coronacrisis. Ik hoop dat de rest van jouw project voorspoedig verloopt.

Mijn vrienden en familie zijn natuurlijk ook belangrijk geweest. Maroesja, Marc en Ineke, fijn dat ik altijd bij jullie terecht kan. Marc, jou wil ik ook bijzonder bedanken voor het ontwerpen van de cover en de hoofdstukpagina's. Na het zien van jouw ontwerp, met de uitleg over de symboliek zag ik het proefschrift ineens voor me. Het betekent veel voor me dat je me hielp en geruststelde. Ma, ik wil u bedanken voor de feedback op mijn Nederlandse samenvatting.

Als laatste wil ik mijn gezin bedanken. Joeri, Nadia en Eline, jullie onvoorwaardelijke liefde helpt mij overal doorheen. Wat fijn dat Nadia en Eline straks als paranimf naast mij kunnen staan. Dirk, als laatste wil ik jou bedanken voor wat je voor me hebt gedaan de afgelopen jaren. Bijvoorbeeld voor het kritisch beluisteren van al mijn presentaties over de verschillende onderzoeken. Je deed dit met het grootste geduld, zelfs als het 
presentaties van bijna een uur waren. Maar het belangrijkste: jouw steun, vertrouwen en trots zijn allesbepalend geweest in het kiezen voor mijn studie en het promotietraject, maar zeker ook voor het afsluiten van dit traject. Bedankt! 


\section{Curriculum Vitae}

Yvonne van der Zalm was born on June 29th, 1968 in Wassenaar, the Netherlands. After completing high school at the Adelbert College Wassenaar, she got her bachelor's degree in occupational therapy at the Amsterdam University of Applied Sciences. Thereafter she obtained a degree in psychiatric nursing at Rivierduinen Mental Health Institute in Leidschendam and worked as a nurse in mental health care in different inpatient wards in this institute. In 2010 she started the premaster Health Sciences at the Vrije Universiteit in Amsterdam and switched to Utrecht University to finish the master nursing sciences. From 2013 she became a member of the research group Mental Health Nursing of Inholland University of Applied Sciences. In 2015 she started as an external PhDcandidate at the School for Mental Health and Neuroscience, Department of Psychiatry and Neuropsychology at Maastricht University. She was supervised by Prof dr. Jean-Paul Selten and Prof. dr. Iris Sommer and continued working for Rivierduinen Mental health Institute. In 2019 she returned to nursing care and started to work in a Flexible Assertive Community team at Rivierduinen Mental Health Institute in Leidschendam. Recently, she started her two-year training to become an advanced nurse practitioner. 
